\title{
SEQUENTIAL FORMULAE FOR THE NORMAL CONE TO SUBLEVEL SETS
}

\author{
A. CABOT AND L. THIBAULT
}

Abstract. Let $X$ be a reflexive Banach space and let $\Phi$ be an extended realvalued lower semicontinuous convex function on $X$. Given a real $\lambda$ and the sublevel set $S=[\Phi \leq \lambda]$, we establish at $\bar{x} \in S$ the following formula for the normal cone to $S$ :

$$
N_{S}(\bar{x})=\limsup _{x \rightarrow \bar{x}} \mathbb{R}_{+} \partial \Phi(x) \quad \text { if } \quad \Phi(\bar{x})=\lambda,
$$

without any qualification condition. The case $\Phi(\bar{x})<\lambda$ is also studied. Here $\mathbb{R}_{+}:=[0,+\infty[$ and $\partial \Phi$ stands for the subdifferential of $\Phi$ in the sense of convex analysis. The proof is based on the sequential convex subdifferential calculus developed previously by the second author. Formula $(\star)$ is extended to nonreflexive Banach spaces via the use of nets. The normal cone to the intersection of finitely many sublevel sets is also examined, thus leading to new formulae without a qualification condition. Our study goes beyond the convex framework: when $\operatorname{dim} X<+\infty$, we show that the inclusion of the left member of $(\star)$ into the right one still holds true for a locally Lipschitz continuous function. Finally, an application of formula $(\star)$ is given to the study of the asymptotic behavior of some gradient dynamical system.

\section{INTRODUCTION}

In classical analysis, it is well known that the gradient of a function $\Phi \in \mathcal{C}^{1}\left(\mathbb{R}^{N}, \mathbb{R}\right)$ at a noncritica 1 point $\bar{x} \in \mathbb{R}^{N}$ is orthogonal to the level surface of $\Phi$ through $\bar{x}$. An analogous result for normals to convex sublevel sets can be stated in terms of subgradients. More precisely, given a real $\lambda \in \mathbb{R}$ and a continuous convex function $\Phi: X \rightarrow \mathbb{R}$ defined on a normed space $X$, let us define the sublevel set $S:=[\Phi \leq \lambda]$. Assuming the Slater condition

$$
[\Phi<\lambda] \neq \emptyset
$$

the normal cone $N_{S}(\bar{x})$ to the set $S$ at $\bar{x} \in S$ is given by

$$
N_{S}(\bar{x})=\mathbb{R}_{+} \partial \Phi(\bar{x}) \quad \text { if } \quad \Phi(\bar{x})=\lambda ;
$$

see for example [4, Proposition 9.6.1] or [8, Lemma V.6]. The subdifferential $\partial \Phi(\bar{x})$ and the normal cone $N_{S}(\bar{x})$ in the sense of convex analysis are recalled at the beginning of the next section. For an extended real-valued convex function $\Phi$ : $\mathbb{R}^{N} \rightarrow \mathbb{R} \cup\{+\infty\}$ such that $\partial \Phi(\bar{x}) \neq \emptyset$, Theorem 23.7 of [31] shows that

$$
N_{S}(\bar{x})=\operatorname{cl}\left(\mathbb{R}_{+} \partial \Phi(\bar{x})\right) \text { if } \Phi(\bar{x})=\lambda,
$$

Received by the editors February 5, 2013 and, in revised form, April 11, 2013.

2010 Mathematics Subject Classification. Primary 90C25, 52A41, 49J52; Secondary 34A60.

Key words and phrases. Convex function, subdifferential, sequential subdifferential calculus, sublevel set, normal cone, nonsmooth analysis.

${ }^{1}$ The terminology of noncritical point means that $\nabla \Phi(\bar{x}) \neq 0$. 
provided that the Slater condition (1.1) is satisfied. Other formulae for $N_{S}(\bar{x})$ are established in the framework of nonsmooth analysis; see for instance $20,28,32$. By particularizing Proposition 10.3 of [32] to the convex case, we obtain that

$$
N_{S}(\bar{x})=\mathbb{R}_{+} \partial \Phi(\bar{x}) \cup N_{\operatorname{dom} \Phi}(\bar{x}) \quad \text { if } \quad \Phi(\bar{x})=\lambda,
$$

for a lower semicontinuous convex function $\Phi: \mathbb{R}^{N} \rightarrow \mathbb{R} \cup\{+\infty\}$ satisfying the Slater condition (1.1). Observe that the assumption $\partial \Phi(\bar{x}) \neq \emptyset$ has been dropped in formula (1.4). However, the common point of formulae (1.2)-(1.4) is that they may fail to be true if the qualification condition (1.1) is not satisfied.

The first aim of the present paper is to establish, without any qualification condition, a general description of $N_{S}(\bar{x})$ in terms of the subdifferential of $\Phi$. For an extended real-valued lower semicontinuous convex function $\Phi: X \rightarrow \mathbb{R} \cup\{+\infty\}$ defined on a reflexive Banach space $X$, we show the following formula:

$$
N_{S}(\bar{x})=\left\{\begin{array}{lll}
\limsup _{x \rightarrow \bar{x}} \mathbb{R}_{+} \partial \Phi(x) & \text { if } & \Phi(\bar{x})=\lambda, \\
\limsup _{x \rightarrow \bar{x}, \mu \downarrow 0} \mu \partial \Phi(x)=N_{\operatorname{dom} \Phi}(\bar{x}) & \text { if } & \Phi(\bar{x})<\lambda .
\end{array}\right.
$$

The technique of the proof is based on sequential convex subdifferential calculus; see 33 35. These papers were originally motivated by the seminal contributions [3,25]. For other references on sequential convex subdifferential calculus, one may consult [24, 26, 30]. The approach is strong enough to allow us to consider the situation where $S$ is the intersection of finitely many sublevel sets and to study the setting of general nonreflexive Banach spaces. The extension to a nonconvex framework is also examined. It is shown for locally Lipschitz functions that the Mordukhovich limiting normal cone to $S$ at $\bar{x}$ is still included in some upper limit expressed with subgradients of $\Phi$.

In the last part of the paper, we apply formula (1.5) to the investigation of the asymptotic behavior of the following dynamical system:

$$
\ddot{x}(t)+\gamma(t) \dot{x}(t)+\partial \Phi(x(t)) \ni 0, \quad t \geq 0,
$$

where $\gamma \in \mathcal{C}\left(\mathbb{R}_{+}, \mathbb{R}_{+}\right)$and $\Phi: \mathbb{R}^{N} \rightarrow \mathbb{R}$ is a convex function with a nonempty set $S$ of minima. The case of a constant coefficient $\gamma>0$ gives rise to the so-called "Heavy Ball with Friction" system. The asymptotic properties of the HBF equation were intensively studied; see for example [1,6] in the case of a smooth potential and [5, 19] in a nonsmooth framework. The case of a vanishing damping parameter $\gamma(t) \rightarrow 0$ has been recently investigated in [15, 16, for a function $\Phi$ of class $\mathcal{C}^{1}$. See also [18] for a related study in the framework of semilinear hyperbolic equations. If the quantity $\gamma(t)$ tends to 0 too rapidly as $t \rightarrow+\infty$, we show that nonstationary solutions of $(E)$ cannot converge toward a minimum point $\bar{x} \in S$ satisfying the obtuseness condition $-N_{S}(\bar{x}) \subset \operatorname{int}\left(T_{S}(\bar{x})\right) \cup\{0\}$.

The paper is organized as follows. Section 2 is concerned with preliminary results, and among them, sequential convex subdifferential calculus plays a major role. Section 3 is the core of the paper: in a reflexive Banach space, the normal cone $N_{S}(\bar{x})$ to the convex sublevel set $S$ is expressed as an upper limit involving the subdifferential of $\Phi$ at nearby points, as written above. An extension of this formula in nonreflexive Banach spaces is considered in Section 4. The case of a finite intersection of convex sublevel sets is examined in Section 5. The extension to a nonconvex framework is studied in Section 6. Finally, in Section 7, the sequential formula for the normal cone is applied to the asymptotic study of the system $(E)$. 


\section{Generalities and preliminary Results}

In this preliminary section, we gather several results that will be useful in the sequel. Given a normed space $X$, let $f: X \rightarrow \mathbb{R} \cup\{+\infty\}$ be a convex function. The effective domain of $f$ is defined by $\operatorname{dom} f=\{x \in X, f(x)<+\infty\}$. The subdifferential of $f$ at $x \in \operatorname{dom} f$ is the set $\partial f(x)$ defined by

$$
\partial f(x)=\left\{\xi^{*} \in X^{*}, f(y) \geq f(x)+\left\langle\xi^{*}, y-x\right\rangle \text { for every } y \in X\right\} .
$$

Given a set $S \subset X$, we denote by $\delta_{S}$ the indicator function of $S$, i.e., $\delta_{S}(x)=0$ if $x \in S$ and $\delta_{S}(x)=+\infty$ if $x \notin S$. The normal cone of $S$ at $x \in S$ is defined by

$$
N_{S}(x)=\partial \delta_{S}(x)=\left\{\xi^{*} \in X^{*},\left\langle\xi^{*}, y-x\right\rangle \leq 0 \text { for every } y \in S\right\} .
$$

We now recall fundamental results with respect to sequential convex subdifferential calculus. The next one concerns the subdifferential of a sum of convex functions; see [33, Theorem 3], 34, Theorem 2.1] and [35, Theorem 1].

Theorem 2.1. Let $X$ be a reflexive Banach space (resp. a general Banach space) and let $f_{1}, f_{2}: X \rightarrow \mathbb{R} \cup\{+\infty\}$ be two proper lower semicontinuous convex functions. Then, for any $\bar{x} \in \operatorname{dom} f_{1} \cap \operatorname{dom} f_{2}$, one has $x^{*} \in \partial\left(f_{1}+f_{2}\right)(\bar{x})$ if and only if there are sequences (resp. nets) $\left(x_{i, n}\right)_{n}$ in $X$ and $\left(x_{i, n}^{*}\right)_{n}$ in $X^{*}$, for $i=1,2$, with $x_{i, n}^{*} \in \partial f_{i}\left(x_{i, n}\right)$ such that

$$
x^{*}=\lim _{\|\|}\left(x_{1, n}^{*}+x_{2, n}^{*}\right) \quad\left(\text { resp. } x^{*}=\lim _{w^{*}}\left(x_{1, n}^{*}+x_{2, n}^{*}\right)\right)
$$

and such that one of the following properties holds:

(a) $\left(x_{i, n}, f_{i}\left(x_{i, n}\right)\right) \rightarrow\left(\bar{x}, f_{i}(\bar{x})\right),\left\langle x_{i, n}^{*}, x_{i, n}-\bar{x}\right\rangle \rightarrow 0$ and $\left(\left\|x_{1, n}^{*}\right\|+\left\|x_{2, n}^{*}\right\|\right)$ $\left(\left\|x_{1, n}-x_{2, n}\right\|\right) \rightarrow 0$

(b) $x_{i, n} \rightarrow \bar{x}$ and $f_{i}\left(x_{i, n}\right)-\left\langle x_{i, n}^{*}, x_{i, n}-\bar{x}\right\rangle \rightarrow f_{i}(\bar{x})$.

Before giving the corresponding statement for the composition of convex functions, let us recall the notion of a vector-valued convex mapping. Let $Y$ be a Banach space and $Y_{+}$a convex cone of $Y$ inducing a preorder $\preccurlyeq$ on $Y$, defined by $y_{1} \preccurlyeq y_{2}$ if and only if $y_{2}-y_{1} \in Y_{+}$. Let $+\infty$ be an abstract maximal element adjoined to $Y$. A mapping $F: X \rightarrow Y \cup\{+\infty\}$ is said to be convex if for all $x, x^{\prime} \in X$, and $t \in] 0,1[$, one has

$$
F\left(t x+(1-t) x^{\prime}\right) \preccurlyeq t F(x)+(1-t) F\left(x^{\prime}\right) .
$$

The set $\operatorname{dom} F=\{x \in X, F(x) \in Y\}$ is the effective domain of $F, \operatorname{Im} F=F(X)$ is the effective image of $F$ and epi $F=\{(x, y) \in X \times Y, F(x) \preccurlyeq y\}$ is the epigraph of $F$. A function $f: Y \rightarrow \mathbb{R} \cup\{+\infty\}$ is $Y_{+}$-nondecreasing on a subset $\Sigma$ of $Y$ if $f\left(y_{1}\right) \leq f\left(y_{2}\right)$ for all $y_{1}, y_{2} \in \Sigma$ satisfying $y_{1} \preccurlyeq y_{2}$. By convention one puts $f(+\infty)=+\infty$ and $y^{*}(+\infty)=+\infty$ for all $y^{*} \in Y_{+}^{*}:=\left\{y^{*} \in Y^{*}:\left\langle y^{*}, y\right\rangle \geq 0 \forall y \in\right.$ $\left.Y_{+}\right\}$. So, if $f$ is the null linear functional $0_{Y^{*}}$, that is, the zero of $Y^{*}$, then

$$
\left(0_{Y^{*}} \circ F\right)(x)=0 \text { if } x \in \operatorname{dom} F \quad \text { and } \quad\left(0_{Y^{*}} \circ F\right)(x)=+\infty \text { if } x \notin \operatorname{dom} F \text {; }
$$

otherwise stated, $0_{Y^{*}} \circ F=\delta_{\operatorname{dom} F}$, the indicator of $\operatorname{dom} F$. In particular, if $Y=\mathbb{R}$, $Y_{+}=\left[0,+\infty\left[\right.\right.$ and $F: X \rightarrow \mathbb{R} \cup\{+\infty\}$, we have $0 F=\delta_{\operatorname{dom} F}$, or in other words, $0(+\infty):=+\infty$. If $f$ is convex and $Y_{+}$-nondecreasing over $\operatorname{Im} F+Y_{+}$, then $f \circ F$ is convex. The following result gives the subdifferential of the composition of a vector-valued convex function with a nondecreasing convex function. The assertion (a) with $\left(i i_{a}\right)$ of the following theorem has been proved in [34. Below we show how the proof of [34, Theorem 3.1] can be adapted to a more general framework. 
Theorem 2.2. Let $X$ and $Y$ be two normed spaces and $Y_{+}$be a convex cone of $Y$. Assume that $F: X \rightarrow Y \cup\{+\infty\}$ is a convex mapping with closed epigraph and that $f: Y \rightarrow \mathbb{R} \cup\{+\infty\}$ is a proper lower semicontinuous convex function which is nondecreasing over $\operatorname{Im} F+Y_{+}$. Let $\bar{x} \in \operatorname{dom}(f \circ F)$.

(a) If $X$ and $Y$ are reflexive Banach spaces, then $x^{*} \in \partial(f \circ F)(\bar{x})$ if and only if there exist sequences $x_{n} \rightarrow \bar{x}, y_{n} \rightarrow F(\bar{x}), x_{n}^{*} \rightarrow x^{*}, e_{n}^{*} \rightarrow 0$, and $y_{n}^{*} \in Y_{+}^{*}$ such that

$$
y_{n}^{*}+e_{n}^{*} \in \partial f\left(y_{n}\right) \quad \text { and } \quad x_{n}^{*} \in \partial\left(y_{n}^{*} \circ F\right)\left(x_{n}\right)
$$

and such that either $\left(i_{a}\right)$ or $\left(i i_{a}\right)$ holds:

$\left(i_{a}\right) f\left(y_{n}\right) \rightarrow f(F(\bar{x})),\left\langle y_{n}^{*}, y_{n}-F(\bar{x})\right\rangle \rightarrow 0,\left\langle y_{n}^{*}, F\left(x_{n}\right)-F(\bar{x})\right\rangle \rightarrow 0$, and $\left\|y_{n}^{*}\right\|\left\|y_{n}-y_{n}^{\prime}\right\| \rightarrow 0$ for some $y_{n}^{\prime} \in F\left(x_{n}\right)+Y_{+}$with $y_{n}^{\prime} \rightarrow F(\bar{x})$ and $\left\langle y_{n}^{*}, y_{n}^{\prime}\right\rangle=\left\langle y_{n}^{*}, F\left(x_{n}\right)\right\rangle$;

$\left(i i_{a}\right) f\left(y_{n}\right)-\left\langle y_{n}^{*}, y_{n}-F(\bar{x})\right\rangle \rightarrow f(F(\bar{x}))$ and $\left\langle y_{n}^{*}, F\left(x_{n}\right)-F(\bar{x})\right\rangle \rightarrow 0$.

(b) If $X$ and $Y$ are general Banach spaces, then $x^{*} \in \partial(f \circ F)(\bar{x})$ if and only if there exist nets $x_{n} \rightarrow \bar{x}, y_{n} \rightarrow F(\bar{x}), x_{n}^{*} \stackrel{w^{*}}{\rightarrow} x^{*}, e_{n}^{*} \stackrel{w^{*}}{\rightarrow} 0$, and $y_{n}^{*} \in Y_{+}^{*}$ such that

$$
y_{n}^{*}+e_{n}^{*} \in \partial f\left(y_{n}\right) \quad \text { and } \quad x_{n}^{*} \in \partial\left(y_{n}^{*} \circ F\right)\left(x_{n}\right)
$$

and such that either $\left(i_{b}\right)$ or $\left(i_{i}\right)$ holds:

$\left(i_{b}\right) f\left(y_{n}\right) \rightarrow f(F(\bar{x})),\left\langle y_{n}^{*}+e_{n}^{*}, y_{n}-F(\bar{x})\right\rangle \rightarrow 0,\left\langle y_{n}^{*}, F\left(x_{n}\right)-F(\bar{x})\right\rangle-$ $\left\langle x_{n}^{*}, x_{n}-\bar{x}\right\rangle \rightarrow 0,\left(\left\|y_{n}^{*}\right\|+\left\|e_{n}^{*}\right\|\right)\left\|y_{n}-y_{n}^{\prime}\right\| \rightarrow 0$ for some $y_{n}^{\prime} \in F\left(x_{n}\right)+Y_{+}$ with $y_{n}^{\prime} \rightarrow F(\bar{x})$ and $\left\langle y_{n}^{*}, y_{n}^{\prime}\right\rangle=\left\langle y_{n}^{*}, F\left(x_{n}\right)\right\rangle$;

(ii $\left.i_{b}\right) f\left(y_{n}\right)-\left\langle y_{n}^{*}+e_{n}^{*}, y_{n}-F(\bar{x})\right\rangle \rightarrow f(F(\bar{x}))$ and $\left\langle y_{n}^{*}, F\left(x_{n}\right)-F(\bar{x})\right\rangle-$ $\left\langle x_{n}^{*}, x_{n}-\bar{x}\right\rangle \rightarrow 0$.

(c) If $X$ is a general Banach space and $Y$ is finite dimensional, then $x^{*} \in$ $\partial(f \circ F)(\bar{x})$ if and only if there exist nets $x_{n} \rightarrow \bar{x}, y_{n} \rightarrow F(\bar{x}), x_{n}^{*} \stackrel{w^{*}}{\rightarrow} x^{*}$, $e_{n}^{*} \rightarrow 0$, and $y_{n}^{*} \in Y_{+}^{*}$ such that

$$
y_{n}^{*}+e_{n}^{*} \in \partial f\left(y_{n}\right) \quad \text { and } \quad x_{n}^{*} \in \partial\left(y_{n}^{*} \circ F\right)\left(x_{n}\right)
$$

and such that either $\left(i_{c}\right)$ or $\left(i i_{c}\right)$ holds:

$\left(i_{c}\right) f\left(y_{n}\right) \rightarrow f(F(\bar{x})),\left\langle y_{n}^{*}, y_{n}-F(\bar{x})\right\rangle \rightarrow 0,\left\langle y_{n}^{*}, F\left(x_{n}\right)-F(\bar{x})\right\rangle \rightarrow 0$, $\left\langle x_{n}^{*}, x_{n}-\bar{x}\right\rangle \rightarrow 0,\left\|y_{n}^{*}\right\|\left\|y_{n}-y_{n}^{\prime}\right\| \rightarrow 0$ for some $y_{n}^{\prime} \in F\left(x_{n}\right)+Y_{+}$with $y_{n}^{\prime} \rightarrow F(\bar{x})$ and $\left\langle y_{n}^{*}, y_{n}^{\prime}\right\rangle=\left\langle y_{n}^{*}, F\left(x_{n}\right)\right\rangle$;

(ii $\left.i_{c}\right) f\left(y_{n}\right)-\left\langle y_{n}^{*}, y_{n}-F(\bar{x})\right\rangle \rightarrow f(F(\bar{x})),\left\langle y_{n}^{*}, F\left(x_{n}\right)-F(\bar{x})\right\rangle \rightarrow 0$, and $\left\langle x_{n}^{*}, x_{n}-\bar{x}\right\rangle \rightarrow 0$.

Proof. Set $\bar{y}:=F(\bar{x})$ and, as usual, also set $f_{1}(x, y):=f(y)$ and $f_{2}(x, y):=$ $\delta_{\text {epi } F}(x, y)$ for all $x \in X$ and $y \in Y$. It is easily checked that $x^{*} \in \partial(f \circ F)(\bar{x})$ if and only if $\left(x^{*}, 0\right) \in \partial\left(f_{1}+f_{2}\right)(\bar{x}, \bar{y})$. By the theorem above, this amounts to saying, whenever both Banach spaces $X$ and $Y$ are reflexive (resp. either $X$ or $Y$ is nonreflexive), that there exist sequences (resp. nets)

$$
\left(0, y_{n}^{*}+e_{n}^{*}\right)+\left(x_{n}^{*},-y_{n}^{*}\right) \rightarrow\left(x^{*}, 0\right) \text { strongly (resp. weakly-*) }
$$

with

$$
\begin{gathered}
y_{n}^{*}+e_{n}^{*} \in \partial f\left(y_{n}\right),\left(x_{n}^{*},-y_{n}^{*}\right) \in \partial \delta_{\text {epi } F}\left(x_{n}, y_{n}^{\prime}\right), \\
y_{n} \rightarrow \bar{y},\left(x_{n}, y_{n}^{\prime}\right) \rightarrow(\bar{x}, \bar{y}), \\
f\left(y_{n}\right) \rightarrow f(\bar{y}),\left\langle y_{n}^{*}+e_{n}^{*}, y_{n}-\bar{y}\right\rangle \rightarrow 0,
\end{gathered}
$$




$$
\begin{gathered}
\left\langle y_{n}^{*}, y_{n}^{\prime}-\bar{y}\right\rangle-\left\langle x_{n}^{*}, x_{n}-\bar{x}\right\rangle \rightarrow 0, \\
\left(\left\|y_{n}^{*}+e_{n}^{*}\right\|+\left\|x_{n}^{*}\right\|+\left\|y_{n}^{*}\right\|\right)\left\|y_{n}-y_{n}^{\prime}\right\| \rightarrow 0 .
\end{gathered}
$$

We observe that (2.2) is equivalent to $x_{n}^{*} \rightarrow x^{*}$ and $e_{n}^{*} \rightarrow 0$ strongly (resp. weakly-*) and that the inclusion $\left(x_{n}^{*},-y_{n}^{*}\right) \in \partial \delta_{\text {epi } F}\left(x_{n}, y_{n}^{\prime}\right)$ means that

$$
\left\langle x_{n}^{*}, x-x_{n}\right\rangle-\left\langle y_{n}^{*}, y-y_{n}^{\prime}\right\rangle \leq 0 \quad \text { for all }(x, y) \in \text { epi } F \text {. }
$$

On one hand, for any $y^{\prime} \in Y_{+}$, taking in the latter inequality $x=x_{n}$ and $y=y_{n}^{\prime}+y^{\prime}$ yields $\left\langle y_{n}^{*}, y^{\prime}\right\rangle \geq 0$ thus $y_{n}^{*} \in Y_{+}^{*}$. On the other hand, taking $x=x_{n}$ and $y=F\left(x_{n}\right)$ in (2.7) we obtain $\left\langle y_{n}^{*}, y_{n}^{\prime}-F\left(x_{n}\right)\right\rangle \leq 0$, and hence $\left\langle y_{n}^{*}, y_{n}^{\prime}-F\left(x_{n}\right)\right\rangle=0$ since $y_{n}^{*} \in Y_{+}^{*}$ and $y_{n}^{\prime}-F\left(x_{n}\right) \in Y_{+}$. Consequently, (2.7) can be rewritten as

$$
\left\langle x_{n}^{*}, x-x_{n}\right\rangle \leq\left\langle y_{n}^{*}, y\right\rangle-\left\langle y_{n}^{*}, F\left(x_{n}\right)\right\rangle \quad \text { for all }(x, y) \in \text { epi } F
$$

which, according to the inclusion $y_{n}^{*} \in Y_{+}^{*}$, is equivalent to

$$
\left\langle x_{n}^{*}, x-x_{n}\right\rangle \leq y_{n}^{*} \circ F(x)-y_{n}^{*} \circ F\left(x_{n}\right) \quad \text { for all } x \in \operatorname{dom} F \text {. }
$$

The function $y_{n}^{*} \circ F$ being convex (since $y_{n}^{*} \in Y_{+}^{*}$ ), the inequality (2.7) is then equivalent to $x_{n}^{*} \in \partial\left(y_{n}^{*} \circ F\right)\left(x_{n}\right)$.

(a) Suppose first that $X$ and $Y$ are reflexive Banach spaces, so the properties above hold true for sequences $\left(x_{n}\right)_{n},\left(x_{n}^{*}\right)_{n}$, etc. Since $x_{n}^{*}\|\| x^{*}$, we observe that (2.5) is equivalent to $\left\langle y_{n}^{*}, y_{n}^{\prime}-\bar{y}\right\rangle \rightarrow 0$, which is equivalent to $\left\langle y_{n}^{*}, F\left(x_{n}\right)-\bar{y}\right\rangle \rightarrow 0$ thanks to the equality $\left\langle y_{n}^{*}, y_{n}^{\prime}-F\left(x_{n}\right)\right\rangle=0$. Further, since $e_{n}^{*} \stackrel{\|}{\rightarrow} 0$, the second convergence in (2.4) is equivalent to $\left\langle y_{n}^{*}, y_{n}-\bar{y}\right\rangle \rightarrow 0$, and the convergence in (2.6) is equivalent to $\left\|y_{n}^{*}\right\|\left\|y_{n}-y_{n}^{\prime}\right\| \rightarrow 0$. So, the conditions in $(a)$ with $\left(i_{a}\right)$ hold true and $\left(i_{a}\right)$ obviously entails $\left(i i_{a}\right)$.

On the other hand, taking sequences as given by $(a)$ with $\left(i i_{a}\right)$, we have, for each $x \in \operatorname{dom} F$,

$$
\begin{aligned}
\left\langle x_{n}^{*}, x-x_{n}\right\rangle \leq & y_{n}^{*}(F(x))-y_{n}^{*}\left(F\left(x_{n}\right)\right) \\
= & \left\langle y_{n}^{*}+e_{n}^{*}, F(x)-y_{n}\right\rangle-\left\langle e_{n}^{*}, F(x)-y_{n}\right\rangle+\left\langle y_{n}^{*}, y_{n}-\bar{y}\right\rangle \\
& -\left\langle y_{n}^{*}, F\left(x_{n}\right)-\bar{y}\right\rangle \\
\leq & f(F(x))-\left\{f\left(y_{n}\right)-\left\langle y_{n}^{*}, y_{n}-\bar{y}\right\rangle\right\}-\left\langle e_{n}^{*}, F(x)-y_{n}\right\rangle \\
& -\left\langle y_{n}^{*}, F\left(x_{n}\right)-\bar{y}\right\rangle,
\end{aligned}
$$

and passing to the limit gives $\left\langle x^{*}, x-\bar{x}\right\rangle \leq f(F(x))-f(F(\bar{x}))$, and hence $x^{*} \in$ $\partial(f \circ F)(\bar{x})$. The equivalences in assertion $(a)$ are then established.

(b) Suppose that $X$ and $Y$ are general Banach spaces. Consider the nets obtained in the part preceding the arguments of $(a)$. From (2.6) we deduce $\left(\left\|y_{n}^{*}\right\|+\left\|e_{n}^{*}\right\|\right)$ $\left\|y_{n}^{\prime}-y_{n}\right\| \rightarrow 0$. Further, since $\left\langle y_{n}^{*}, y_{n}^{\prime}-F\left(x_{n}\right)\right\rangle=0$, it results from (2.5) that $\left\langle y_{n}^{*}, F\left(x_{n}\right)-F(\bar{x})\right\rangle-\left\langle x_{n}^{*}, x_{n}-\bar{x}\right\rangle \rightarrow 0$. All the properties in $(b)$ with $\left(i_{b}\right)$ are then justified and the ones concerning $\left(i i_{b}\right)$ readily follow. 
Now let us show that $(b)$ with $\left(i i_{b}\right)$ implies $x^{*} \in \partial(f \circ F)(\bar{x})$. Considering the nets as given by $(b)$ with $\left(i i_{b}\right)$, we have, for each $x \in \operatorname{dom} F$,

$$
\begin{aligned}
\left\langle x_{n}^{*}, x-\bar{x}\right\rangle= & \left\langle x_{n}^{*}, x-x_{n}\right\rangle+\left\langle x_{n}^{*}, x_{n}-\bar{x}\right\rangle \\
\leq & y_{n}^{*} \circ F(x)-y_{n}^{*} \circ F\left(x_{n}\right)+\left\langle x_{n}^{*}, x_{n}-\bar{x}\right\rangle \\
= & \left\langle y_{n}^{*}+e_{n}^{*}, F(x)-y_{n}\right\rangle-\left\langle e_{n}^{*}, F(x)-\bar{y}\right\rangle+\left\langle y_{n}^{*}+e_{n}^{*}, y_{n}-\bar{y}\right\rangle \\
& \quad-\left\langle y_{n}^{*}, F\left(x_{n}\right)-\bar{y}\right\rangle+\left\langle x_{n}^{*}, x_{n}-\bar{x}\right\rangle \\
\leq & f(F(x))-\left\{f\left(y_{n}\right)-\left\langle y_{n}^{*}+e_{n}^{*}, y_{n}-\bar{y}\right\rangle\right\}-\left\langle e_{n}^{*}, F(x)-\bar{y}\right\rangle \\
& \quad-\left\{\left\langle y_{n}^{*}, F\left(x_{n}\right)-\bar{y}\right\rangle-\left\langle x_{n}^{*}, x_{n}-\bar{x}\right\rangle\right\},
\end{aligned}
$$

and this gives $\left\langle x^{*}, x-\bar{x}\right\rangle \leq f(F(x))-f(F(\bar{x}))$, and hence $x^{*} \in \partial(f \circ F)(\bar{x})$. The equivalences in assertion $(b)$ are then proved.

$(c)$ Consider the nets given by $(b)$ with $\left(i_{b}\right)$. From the finite dimensional property of $Y$ the net $\left(e_{n}^{*}\right)_{n}$ strongly converges to zero. The convergence $\left\langle y_{n}^{*}+e_{n}^{*}, y_{n}-\bar{y}\right\rangle \rightarrow 0$ from $\left(i_{b}\right)$ then ensures $\left\langle y_{n}^{*}, y_{n}-\bar{y}\right\rangle \rightarrow 0$. Combining this with the convergence $\left\|y_{n}^{*}\right\|\left\|y_{n}^{\prime}-y_{n}\right\| \rightarrow 0$ and with the equality $\left\langle y_{n}^{*}, y_{n}^{\prime}\right\rangle=\left\langle y_{n}^{*}, F\left(x_{n}\right)\right\rangle$ from $\left(i_{b}\right)$, we obtain $\left\langle y_{n}^{*}, F\left(x_{n}\right)-\bar{y}\right\rangle \rightarrow 0$. It results from this and from the convergence $\left\langle y_{n}^{*}, F\left(x_{n}\right)-\right.$ $\bar{y}\rangle-\left\langle x_{n}^{*}, x_{n}-\bar{x}\right\rangle \rightarrow 0$ that $\left\langle x_{n}^{*}, x_{n}-\bar{x}\right\rangle \rightarrow 0$. All the properties in $\left(i_{c}\right)$ are then deduced from the ones in $\left(i_{b}\right)$, and evidently $\left(i i_{c}\right)$ follows from $\left(i_{c}\right)$.

Finally, as in $(a)$ above, one also shows that $(c)$ with $\left(i i_{c}\right)$ entails that $x^{*} \in$ $\partial(f \circ F)(\bar{x})$.

By taking a continuous affine mapping $F$ and the cone $Y_{+}=\{0\}$, the above theorem implies the following statement; see [34, Corollary 3.3].

Corollary 2.3. Let $X$ and $Y$ be two reflexive Banach spaces and $F: X \rightarrow Y$ be a continuous affine mapping with linear part $A \in \mathcal{L}(X, Y)$. Suppose that $f: Y \rightarrow \mathbb{R} \cup$ $\{+\infty\}$ is a proper convex lower semicontinuous function. Then for $\bar{x} \in \operatorname{dom}(f \circ F)$ one has $x^{*} \in \partial(f \circ F)(\bar{x})$ if and only if there exist

$$
\begin{gathered}
y_{n}^{*} \in \partial f\left(y_{n}\right) \quad \text { with } \quad y_{n}^{*} \circ A \rightarrow x^{*}, \\
y_{n} \rightarrow F(\bar{x}) \quad \text { and } \quad f\left(y_{n}\right)-\left\langle y_{n}^{*}, y_{n}-F(\bar{x})\right\rangle \rightarrow f(F(\bar{x})) .
\end{gathered}
$$

Let us now recall the notion of Painlevé-Kuratowski limits. Given a subset $T$ of the normed space $X$, a set-valued mapping $M: T \rightrightarrows Z$ from $T$ into a topological space $Z$, and $\bar{x} \in \operatorname{cl}_{X}(T)$, we recall that the upper and lower limits as $T \ni x \rightarrow \bar{x}$ are defined by

$$
\limsup _{T \ni x \rightarrow \bar{x}} M(x):=\left\{z \in Z: \forall W \in \mathcal{N}_{Z}(z), \forall V \in \mathcal{N}_{X}(\bar{x}), \exists x \in V \cap T, M(x) \cap W \neq \emptyset\right\}
$$

and

$$
\liminf _{T \ni x \rightarrow \bar{x}} M(x):=\left\{z \in Z: \forall W \in \mathcal{N}_{Z}(z), \exists V \in \mathcal{N}_{X}(\bar{x}), \forall x \in V \cap T, M(x) \cap W \neq \emptyset\right\} .
$$

Above $\mathcal{N}_{Z}(z)$ stands for the set of neighborhoods of $z$ in $Z$. If both semilimits coincide, the common set is called the Painlevé-Kuratowski limit of $M(x)$ as $T \ni$ $x \rightarrow \bar{x}$ and one writes $\lim _{T \ni x \rightarrow \bar{x}} M(x)$.

It is known that $z \in \lim \sup _{T \ni x \rightarrow \bar{x}} M(x)$ if and only if there are a net $\left(x_{i}\right)_{i \in I}$ in $T$ converging to $\bar{x}$ and a net $\left(z_{i}\right)_{i \in I}$ in $Z$ converging to $z$ such that $z_{i} \in M\left(x_{i}\right)$ for all $i \in I$. When the topology of $Z$ is metrizable, $\left(x_{i}\right)_{i}$ and $\left(z_{i}\right)_{i}$ can be taken as sequences. 
One also knows that $z \in \liminf _{T \ni x \rightarrow \bar{x}} M(x)$ if and only if for any net $\left(x_{i}\right)_{i \in I}$ in $T$ converging to $\bar{x}$ there exist a subnet $\left(x_{s(j)}\right)_{j \in J}$ and a net $\left(z_{j}\right)_{j \in J}$ in $Z$ converging to $z$ such that $z_{j} \in M\left(x_{s(j)}\right)$. When the topology of $Z$ is metrizable, it is also necessary and sufficient that for any sequence $\left(x_{n}\right)_{n \in \mathbb{N}}$ in $T$ converging to $\bar{x}$ there exists a sequence $\left(z_{n}\right)_{n \in \mathbb{N}}$ in $Z$ converging to $z$ such that $z_{n} \in M\left(x_{n}\right)$ for all $n$ large enough.

When $Z$ is a normed space $E$ (resp. the topological dual of $E$ ), that is, $Z=E$ (resp. $Z=E^{*}$ ), both strong and weak (resp. weak-star) topologies on $E$ (resp. $E^{*}$ ) can be considered. As said above, only sequences are needed to describe the upper and lower limits whenever $E$ (resp. $E^{*}$ ) is endowed with the norm-topology, but nets are required provided that $E$ (resp. $E^{*}$ ) is equipped with the weak (resp. weak-star) topology. Below, the upper and lower limits with respect to the strong topology of $E$ (resp. $E^{*}$ ) are denoted by $\lim \sup _{T \ni x \rightarrow \bar{x}} M(x)$ and $\lim \inf _{T \ni x \rightarrow \bar{x}} M(x)$, and those limits with respect to the weak (resp. weak-star) topology of $E$ (resp. $E^{*}$ ) are denoted respectively by

$$
{ }^{w} \limsup _{T \ni x \rightarrow \bar{x}} M(x),{ }^{w} \liminf _{T \ni x \rightarrow \bar{x}} M(x) \quad\left(\text { resp. }{ }^{w^{*}} \limsup _{T \ni x \rightarrow \bar{x}} M(x),{ }^{w^{*}} \liminf _{T \ni x \rightarrow \bar{x}} M(x)\right) .
$$

Sometimes, even for $E$ (resp. $E^{*}$ ) endowed with the weak (resp. weak-star) topology, one needs to work only with the above sequential convergence properties. Otherwise stated, for $E$ (resp. $E^{*}$ ) endowed with the weak (resp. weak-star) topology, one needs to consider the sequential upper and lower limits

$$
{ }^{\text {seq }} \limsup _{T \ni x \rightarrow \bar{x}} M(x) \quad \text { and } \quad \text { seq } \liminf _{T \ni x \rightarrow \bar{x}} M(x)
$$

defined as follows: An element $u \in E$ (resp. $\left.u^{*} \in E^{*}\right)$ belongs to ${ }^{\text {seq }} \lim \sup _{T \ni x \rightarrow \bar{x}} M(x)$ provided there exist sequences $\left(x_{n}\right)_{n \in \mathbb{N}}$ in $T$ converging strongly to $\bar{x}$ and $\left(u_{n}\right)_{n \in \mathbb{N}}$ in $E$ (resp. $\left(u_{n}^{*}\right)_{n \in \mathbb{N}}$ in $\left.E^{*}\right)$ converging weakly (resp. weakly star) to $u$ (resp. $u^{*}$ ) with $u_{n} \in M\left(x_{n}\right)$ (resp. $u_{n}^{*} \in M\left(x_{n}\right)$ ) for all $n \in \mathbb{N}$; similarly, the sequential lower limit ${ }^{\text {seq }} \lim \inf _{T \ni x \rightarrow \bar{x}} M(x)$ is the set of $u \in E$ (resp. $\left.u^{*} \in E^{*}\right)$ such that for any sequence $\left(x_{n}\right)_{n \in \mathbb{N}}$ in $T$ converging strongly to $\bar{x}$ there exists a sequence $\left(u_{n}\right)_{n \in \mathbb{N}}$ in $E$ (resp. $\left(u_{n}^{*}\right)_{n \in \mathbb{N}}$ in $\left.E^{*}\right)$ converging weakly (resp. weakly star) to $u$ (resp. $u^{*}$ ) with $u_{n} \in M\left(x_{n}\right)$ (resp. $\left.u_{n}^{*} \in M\left(x_{n}\right)\right)$ for all $n$ large enough.

In the case of a sequence of sets $\left(C_{n}\right) \subset X$, the upper and lower limits are then translated as

$$
\limsup _{n \rightarrow+\infty} C_{n}=\left\{x \in X, \exists n_{k} \rightarrow+\infty, \exists x_{n_{k}} \in C_{n_{k}} \text { with } x_{n_{k}} \underset{k \rightarrow+\infty}{\longrightarrow} x\right\}
$$

and

$$
\liminf _{n \rightarrow+\infty} C_{n}=\left\{x \in X, \exists x_{n} \in C_{n} \text { for large } n, \text { with } x_{n} \underset{n \rightarrow+\infty}{\longrightarrow} x\right\} .
$$

The Painlevé-Kuratowski limit of the sequence $\left(C_{n}\right)$, when the upper and lower limits coincide, is denoted by $\lim _{n \rightarrow+\infty} C_{n}$.

When the sequence $\left(C_{n}\right)_{n \in \mathbb{N}}$ Painlevé-Kuratowski converges to $C$ with respect to both the strong and the weak topologies of $X$, one says that the sequence $\left(C_{n}\right)_{n \in \mathbb{N}}$ Mosco-converges to $C$. It is easily seen that this is equivalent to

$$
\text { seq } \limsup _{n \rightarrow+\infty} C_{n} \subset C \subset \liminf _{n \rightarrow+\infty} C_{n} .
$$


The graph of the set-valued mapping $M: X \rightrightarrows Z$ is defined by

$$
\operatorname{gph} M=\{(x, z) \in X \times Z, z \in M(x)\} .
$$

Let us now state the Attouch theorem (see [2]) applied to the indicator functions of sets.

Theorem 2.4. Let $X$ be a reflexive Banach space. Let $C$ and $C_{n}, n \in \mathbb{N}$, be nonempty closed convex sets of $X$. Assume that the sequence $\left(C_{n}\right)$ converges toward $C$ in the sense of Mosco. Then the mappings $N_{C_{n}}$ converge graphically to $N_{C}$. This means equivalently that

(i) $\limsup _{n \rightarrow+\infty}\left(\operatorname{gph} N_{C_{n}}\right) \subset \operatorname{gph} N_{C}$.

(ii) $\operatorname{gph} N_{C} \subset \liminf _{n \rightarrow+\infty}\left(\operatorname{gph} N_{C_{n}}\right)$.

When the sets $C_{n}$ are sublevel sets, Mosco-convergence of the sequence $\left(C_{n}\right)$ can be easily obtained, as shown by the following statement. In the sequel, we denote by $[\Phi \leq \lambda]$ the sublevel set $\{x \in X, \Phi(x) \leq \lambda\}$.

Lemma 2.5. Let $X$ be a normed space and let $\Phi: X \rightarrow \mathbb{R} \cup\{+\infty\}$ be a lower semicontinuous convex function. Given a sequence $\left(\lambda_{n}\right) \subset \mathbb{R}$, let us define the set $C_{n}$ by $C_{n}=\left[\Phi \leq \lambda_{n}\right]$.

(i) If the sequence $\left(\lambda_{n}\right)$ is nonincreasing and satisfies $\lim _{n \rightarrow+\infty} \lambda_{n}=\lambda \in$ $\mathbb{R} \cup\{-\infty\}$, then the sequence $\left(C_{n}\right)$ Mosco-converges to the set $C=[\Phi \leq \lambda]$.

(ii) If the sequence $\left(\lambda_{n}\right)$ is nondecreasing and satisfies $\lim _{n \rightarrow+\infty} \lambda_{n}=\lambda \in$ $\mathbb{R} \cup\{+\infty\}$ with $C_{n} \neq \emptyset$ for $n$ large enough, then the sequence $\left(C_{n}\right)$ Moscoconverges to the set $C$ defined by

$$
C= \begin{cases}{[\Phi \leq \lambda]} & \text { if } \lambda<+\infty \\ \operatorname{cl}(\operatorname{dom} \Phi) & \text { if } \lambda=+\infty .\end{cases}
$$

Proof. ( $i)$ The sequence $\left(C_{n}\right)$ is nonincreasing, hence

$$
\lim _{n \rightarrow+\infty} C_{n}=\bigcap_{n \in \mathbb{N}} \operatorname{cl}\left(C_{n}\right) \text { and }{ }^{w} \lim _{n \rightarrow+\infty} C_{n}=\bigcap_{n \in \mathbb{N}} \operatorname{cl}_{w}\left(C_{n}\right) .
$$

The sets $C_{n}$ are closed by the lower semicontinuity of $\Phi$. Since the sets $C_{n}$ are convex, they are also weakly closed. It ensues that

$$
\lim _{n \rightarrow+\infty} C_{n}={ }^{w} \lim _{n \rightarrow+\infty} C_{n}=\bigcap_{n \in \mathbb{N}} C_{n}=[\Phi \leq \lambda],
$$

and the sequence $\left(C_{n}\right)$ Mosco-converges to the set $C:=[\Phi \leq \lambda]$.

(ii) If $\lambda_{n_{0}}=\lambda$ for some $n_{0} \in \mathbb{N}$, then $\lambda_{n}=\lambda$ for every $n \geq n_{0}$ and the previous case applies. Hence we can suppose without loss of generality that $\lambda_{n}<\lambda$ for every $n \in \mathbb{N}$. The sequence $\left(C_{n}\right)$ is nondecreasing, hence

$$
\begin{gathered}
\lim _{n \rightarrow+\infty} C_{n}=\operatorname{cl}\left(\bigcup_{n \in \mathbb{N}} C_{n}\right)=\operatorname{cl}([\Phi<\lambda]), \\
{ }^{w} \lim _{n \rightarrow+\infty} C_{n}=\operatorname{cl}_{w}\left(\bigcup_{n \in \mathbb{N}} C_{n}\right)=\operatorname{cl}_{w}([\Phi<\lambda]) .
\end{gathered}
$$

Since the set $[\Phi<\lambda]$ is convex, we have $\operatorname{cl}([\Phi<\lambda])=\operatorname{cl}_{w}([\Phi<\lambda])$. It ensues that the sequence $\left(C_{n}\right)$ Mosco-converges to the set $C:=\operatorname{cl}([\Phi<\lambda])$. If $\lambda=+\infty$, 
we have $C=\operatorname{cl}(\operatorname{dom} \Phi)$. If $\lambda<+\infty$, taking $n_{0} \in \mathbb{N}$ with $C_{n_{0}} \neq \emptyset$ we observe that $[\Phi<\lambda] \supset C_{n_{0}} \neq \emptyset$, so we can fix $a \in[\Phi<\lambda]$. For any $x \in[\Phi \leq \lambda]$, we have

$$
\Phi\left(\frac{1}{n} a+\left(1-\frac{1}{n}\right) x\right) \leq \frac{1}{n} \Phi(a)+\left(1-\frac{1}{n}\right) \Phi(x)<\lambda ;
$$

hence $\frac{1}{n} a+\left(1-\frac{1}{n}\right) x \in[\Phi<\lambda]$, which entails $x \in \mathrm{cl}([\Phi<\lambda])$. Hence we have proved that $[\Phi \leq \lambda] \subset \operatorname{cl}([\Phi<\lambda])$. Since the reverse inclusion follows from the lower semicontinuity of $\Phi$, we infer that $C=\operatorname{cl}([\Phi<\lambda])=[\Phi \leq \lambda]$ when $\lambda<+\infty$. The proof is complete.

Let us end these preliminary results with the notion of a horizon subdifferential. Let $X$ be a normed space and let $\Phi: X \rightarrow \mathbb{R} \cup\{+\infty\}$ be a lower semicontinuous convex function. The horizon subdifferential of $\Phi$ at $\bar{x} \in \operatorname{dom} \Phi$ is defined by

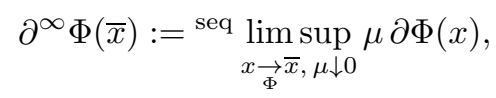

that is, $x^{*} \in \partial^{\infty} \Phi(\bar{x})$ provided there exist sequences $\left(x_{n}\right) \subset X,\left(x_{n}^{*}\right) \subset X^{*},\left(\mu_{n}\right) \downarrow 0$ such that $x_{n} \rightarrow \bar{x}, \Phi\left(x_{n}\right) \rightarrow \Phi(\bar{x}), x_{n}^{*} \in \partial \Phi\left(x_{n}\right)$ and $\mu_{n} x_{n}^{*} \stackrel{w^{*}}{\rightarrow} x^{*}$.

Lemma 2.6. Let $X$ be a Banach space and let $\Phi: X \rightarrow \mathbb{R} \cup\{+\infty\}$ be a proper lower semicontinuous convex function. Then we have, for every $\bar{x} \in \operatorname{dom} \Phi$,

$$
\begin{aligned}
\partial^{\infty} \Phi(\bar{x}) & =\limsup _{x \rightarrow \bar{\Phi}} \mu \partial \downarrow \Phi(x)=\limsup _{x \rightarrow \bar{x}, \mu \downarrow 0} \mu \partial \Phi(x)={ }^{\text {seq }} \limsup _{x \rightarrow \bar{x}, \mu \downarrow 0} \mu \partial \Phi(x) \\
& =N_{\operatorname{dom} \Phi}(\bar{x})=\left\{x^{*} \in X^{*}:\left(x^{*}, 0\right) \in N_{\text {epi } f}((\bar{x}, f(\bar{x})))\right\} .
\end{aligned}
$$

Proof. Consider any $\bar{x} \in \operatorname{dom} \Phi$. The inclusions of the second member into the third and the third into the fourth are obvious. Let us show that the fourth member is included into the fifth, that is,

$$
{ }^{\text {seq }} \limsup _{x \rightarrow \bar{x}, \mu \downarrow 0} \mu \partial \Phi(x) \subset N_{\text {dom } \Phi}(\bar{x}) .
$$

Let $x^{*} \in{ }^{\text {seq }} \limsup _{x \rightarrow \bar{x}, \mu \downarrow 0} \mu \partial \Phi(x)$. By definition, there exist $\left(x_{n}\right) \subset X,\left(x_{n}^{*}\right) \subset X^{*}$ and $\left(\mu_{n}\right) \downarrow 0$ such that $x_{n} \rightarrow \bar{x}, x_{n}^{*} \in \partial \Phi\left(x_{n}\right)$ and $\mu_{n} x_{n}^{*} \stackrel{w^{*}}{\rightarrow} x^{*}$. Take a real $\varepsilon>0$ and by the lower semicontinuity of $\Phi$ choose some integer $n_{0}$ such that $\Phi(\bar{x})-\varepsilon \leq \Phi\left(x_{n}\right)$ for all $n \geq n_{0}$. Fixing any $x \in \operatorname{dom} \Phi$, the subdifferential inequality implies that, for every $n \geq n_{0}$,

$$
\left\langle\mu_{n} x_{n}^{*}, x-x_{n}\right\rangle \leq \mu_{n}\left(\Phi(x)-\Phi\left(x_{n}\right)\right) \leq \mu_{n}(\Phi(x)-\Phi(\bar{x})+\varepsilon) .
$$

Taking the limit as $n \rightarrow+\infty$ in both extreme members of (2.9), we find $\left\langle x^{*}, x-\bar{x}\right\rangle$ $\leq 0$ because $x_{n} \rightarrow \bar{x}, \mu_{n} x_{n}^{*} \stackrel{w^{*}}{\rightarrow} x^{*}$ and $\mu_{n} \rightarrow 0$ as $n \rightarrow+\infty$. Since this is true for every $x \in \operatorname{dom} \Phi$, we infer that $x^{*} \in N_{\operatorname{dom} \Phi}(\bar{x})$, which shows the inclusion (2.8).

To prove the inclusion of the fifth member into the second, fix any $x^{*} \in N_{\operatorname{dom} \Phi}(\bar{x})$. For each real $\eta>0$, we know from the lower semicontinuity of the convex function $\Phi$ (see, e.g., [8] or [23]) that $\partial_{\eta^{2}} \Phi(\bar{x}) \neq \emptyset$, so we choose some $y_{\eta}^{*} \in \partial_{\eta^{2}} \Phi(\bar{x})$. Putting $\mu(\eta):=\eta /\left(1+\left\|y_{\eta}^{*}\right\|\right)$, it is clear from the definitions of an approximate subdifferential and a normal cone that $z_{\eta}^{*}:=y_{\eta}^{*}+\mu(\eta)^{-1} x^{*} \in \partial_{\eta^{2}} \Phi(\bar{x})$. Since $X$ is a Banach space, the Borwein version 2 of the Brønsted-Rockafellar theorem [14] yields some $x_{\eta} \in X$

\footnotetext{
${ }^{2}$ See [10, Theorem 1] or also [8] Theorem IV.52].
} 
and $x_{\eta}^{*} \in \partial \Phi\left(x_{\eta}\right)$ such that

$$
\left\|x_{\eta}-\bar{x}\right\| \leq \eta,\left|\Phi\left(x_{\eta}\right)-\Phi(\bar{x})\right| \leq \eta(\eta+1),\left\|x_{\eta}^{*}-z_{\eta}^{*}\right\| \leq \eta\left(1+\left\|z_{\eta}^{*}\right\|\right) .
$$

Observing that the latter inequality entails

$$
\left\|\mu(\eta) x_{\eta}^{*}-\mu(\eta) y_{\eta}^{*}-x^{*}\right\| \leq \eta\left(\mu(\eta)+\mu(\eta)\left\|y_{\eta}^{*}\right\|+\left\|x^{*}\right\|\right)
$$

and noting that $\mu(\eta)\left\|y_{\eta}^{*}\right\| \leq \eta \rightarrow 0$ as $\eta \downarrow 0$, we see that $\mu(\eta) x_{\eta}^{*} \rightarrow x^{*}$ with $\mu(\eta) \downarrow 0$ as $\eta \downarrow 0$. This justifies the desired inclusion $N_{\operatorname{dom} \Phi}(\bar{x}) \subset \limsup _{x \rightarrow \underset{\Phi}{x}, \mu \downarrow 0} \mu \partial \Phi(x)$. So, we have established the equality between the second, third, fourth and fifth members. Further, the second member is obviously included into the first and the first is also obviously included into the fourth, so we can add the first member into the preceding chain of equalities.

Finally, the equality $N_{\operatorname{dom} \Phi}(\bar{x})=\left\{x^{*}:\left(x^{*}, 0\right) \in N_{\text {epi } \Phi}((\bar{x}, \Phi(\bar{x})))\right\}$ follows easily from the definition of a normal cone to a convex set, so the proof of the lemma is completed.

\section{A SEQUential Formula For the normals to SUblevel SETS}

Let $X$ be a reflexive Banach space and let $\Phi: X \rightarrow \mathbb{R} \cup\{+\infty\}$ be a lower semicontinuous convex function. In the next theorem, we establish a sequential formula for the normal cone to the sublevel set, which is valid without any qualification condition. For convenience, throughout the remainder of the paper we denote as usual $\left.\left.\mathbb{R}_{-}:=\right]-\infty, 0\right]$ and $\mathbb{R}_{+}:=[0,+\infty[$.

Theorem 3.1. Let $X$ be a reflexive Banach space and let $\Phi: X \rightarrow \mathbb{R} \cup\{+\infty\}$ be a lower semicontinuous convex function. Given $\lambda \in \mathbb{R}$, let us define the set $S$ by $S=[\Phi \leq \lambda]$. If $\bar{x} \in S$, we have

$$
\begin{aligned}
N_{S}(\bar{x})= & \limsup \left\{\begin{array}{l}
x \rightarrow \bar{x}, \mu \geq 0 \\
\mu(\Phi(x)-\bar{\lambda}) \rightarrow 0
\end{array}\right. \\
= & \limsup \quad \mu \partial \Phi(x) \\
& \left\{\begin{array}{l}
x \rightarrow \bar{x}, \mu \geq 0 \\
\mu(\Phi(x)-\bar{\lambda}) \rightarrow 0
\end{array}\right. \\
= & \left\{\begin{array}{l}
\limsup _{x \rightarrow \bar{x}} \mathbb{R}_{+} \partial \Phi(x) \\
\limsup _{x \rightarrow \bar{x}, \mu \downarrow 0} \mu \partial \Phi(x)=N_{\operatorname{dom} \Phi}(\bar{x}) \text { if } \quad \Phi(\bar{x})<\lambda,
\end{array}\right.
\end{aligned}
$$

(Concerning the second member of the first equality we recall that $0 \Phi=\delta_{\mathrm{dom} \Phi}$. )

Proof. Let us start with the inclusion

$$
\begin{aligned}
N_{S}(\bar{x}) \subset & \limsup _{\left\{\begin{array}{l}
x \rightarrow \bar{x}, \mu \geq 0 \\
\mu(\Phi(x)-\lambda) \rightarrow 0
\end{array}\right.} \partial(\mu \Phi)(x) .
\end{aligned}
$$

Define the lower semicontinuous convex function $F: X \rightarrow \mathbb{R} \cup\{+\infty\}$ by $F(x)=$ $\Phi(x)-\lambda$. Observe that $S=\left\{x \in X, F(x) \in \mathbb{R}_{-}\right\}$and hence $\delta_{S}=\delta_{\mathbb{R}_{-}} \circ F$. This implies that

$$
N_{S}(\bar{x})=\partial \delta_{S}(\bar{x})=\partial\left(\delta_{\mathbb{R}_{-}} \circ F\right)(\bar{x}) .
$$

The function $\delta_{\mathbb{R}_{-}}$is lower semicontinuous convex and nondecreasing. Let us fix $x^{*} \in N_{S}(\bar{x})$ and apply Theorem $2.2(a)$ with $Y=\mathbb{R}, Y_{+}=\mathbb{R}_{+}, \quad f=\delta_{\mathbb{R}_{-}}$and the function $F$ defined above. We obtain the existence of $\left(x_{n}\right) \subset X,\left(y_{n}\right) \subset \mathbb{R}$, 
$\left(x_{n}^{*}\right) \subset X^{*},\left(e_{n}^{*}\right) \subset \mathbb{R}$ and $\left(y_{n}^{*}\right) \subset \mathbb{R}_{+}$such that $x_{n} \rightarrow \bar{x}, y_{n} \rightarrow \Phi(\bar{x})-\lambda, x_{n}^{*} \rightarrow x^{*}$, $e_{n}^{*} \rightarrow 0$ and

$$
\begin{gathered}
y_{n}^{*}+e_{n}^{*} \in N_{\mathbb{R}_{-}}\left(y_{n}\right), \\
x_{n}^{*} \in \partial\left(y_{n}^{*} \Phi\right)\left(x_{n}\right), \\
y_{n}^{*}\left(\Phi\left(x_{n}\right)-\Phi(\bar{x})\right) \rightarrow 0 \quad \text { as } n \rightarrow+\infty .
\end{gathered}
$$

Let us assume that $\Phi(\bar{x})<\lambda$. Since $y_{n} \rightarrow \Phi(\bar{x})-\lambda$ as $n \rightarrow+\infty$, we have $y_{n}<0$ for $n$ large enough. It ensues that $N_{\mathbb{R}_{-}}\left(y_{n}\right)=\{0\}$ and formula (3.5) then implies that

$$
y_{n}^{*}=-e_{n}^{*} \rightarrow 0 \quad \text { as } n \rightarrow+\infty, \quad \text { if } \Phi(\bar{x})<\lambda .
$$

Now observe that

$$
y_{n}^{*}\left(\Phi\left(x_{n}\right)-\lambda\right)=y_{n}^{*}\left(\Phi\left(x_{n}\right)-\Phi(\bar{x})\right)+y_{n}^{*}(\Phi(\bar{x})-\lambda) .
$$

In view of (3.7)-(3.8), we immediately deduce that

$$
y_{n}^{*}\left(\Phi\left(x_{n}\right)-\lambda\right) \rightarrow 0 \quad \text { as } n \rightarrow+\infty .
$$

If $\Phi(\bar{x})=\lambda$, formula (3.9) also holds true as a direct consequence of (3.7). Finally, we have built sequences $x_{n} \rightarrow \bar{x}, x_{n}^{*} \rightarrow x^{*},\left(y_{n}^{*}\right) \subset \mathbb{R}_{+}$satisfying (3.6) and (3.9), which clearly shows that

$$
x^{*} \in \limsup _{\left\{\begin{array}{l}
x \rightarrow \bar{x}, \mu \geq 0 \\
\mu(\Phi(x)-\lambda) \rightarrow 0
\end{array}\right.} \partial(\mu \Phi)(x) .
$$

Inclusion (3.4) is proved.

Let us now establish the inclusion

$$
\begin{aligned}
& \limsup _{\left\{\begin{array}{l}
x \rightarrow \mu \geq 0 \\
\mu(\Phi(x)-\lambda) \rightarrow 0
\end{array}\right.} \partial(\mu \Phi)(x) \subset \limsup \quad \mu \partial \Phi(x) . \\
& \mu(\Phi(x)-\bar{\lambda}) \rightarrow 0
\end{aligned}
$$

Let $x^{*} \in \limsup \quad \partial(\mu \Phi)(x)$. By definition, there exist sequences $x_{n} \rightarrow \bar{x}$, $\left\{\begin{array}{l}x \rightarrow \bar{x}, \mu \geq 0 \\ \mu(\Phi(x)-\bar{\lambda}) \rightarrow 0\end{array}\right.$

$x_{n}^{*} \rightarrow x^{*},\left(\mu_{n}\right) \subset \mathbb{R}_{+}$satisfying $x_{n}^{*} \in \partial\left(\mu_{n} \Phi\right)\left(x_{n}\right)$ and $\mu_{n}\left(\Phi\left(x_{n}\right)-\lambda\right) \rightarrow 0$. First assume that there exists a subsequence of $\left(\mu_{n}\right)$, still denoted by $\left(\mu_{n}\right)$, such that $\mu_{n}>0$ for every $n \in \mathbb{N}$. We then have $x_{n}^{*} \in \mu_{n} \partial \Phi\left(x_{n}\right)$, and hence

$$
\begin{aligned}
x^{*} \in & \limsup _{\left\{\begin{array}{l}
x \rightarrow \bar{x}, \mu \geq 0 \\
\mu(\Phi(x)-\lambda) \rightarrow 0
\end{array}\right.} \mu \partial \Phi(x) .
\end{aligned}
$$

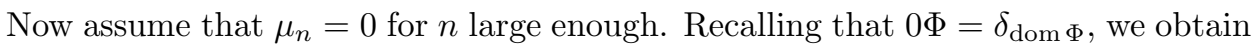
$x_{n}^{*} \in N_{\text {dom } \Phi}\left(x_{n}\right)$ for $n$ large enough. Since the operator $N_{\operatorname{cl}(\operatorname{dom} \Phi)}$ is graph-closed with respect to the norms in $X$ and $X^{*}$, we deduce at the limit as $n \rightarrow+\infty$ that $x^{*} \in N_{\operatorname{dom} \Phi}(\bar{x})$. In view of Lemma 2.6. we have $N_{\operatorname{dom} \Phi}(\bar{x})=\limsup _{x \rightarrow \bar{\Phi}} \mu \partial \downarrow \Phi \Phi(x)$; hence

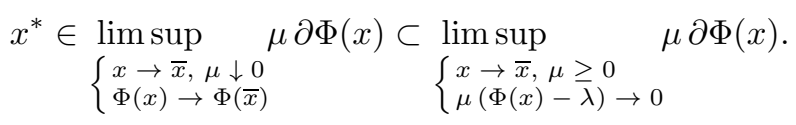

Therefore the inclusion (3.11) is satisfied in both cases, which proves (3.10). 
Let us now show the inclusion

$$
\begin{aligned}
& \limsup \sup _{\substack{x \rightarrow \bar{x}, \mu \geq 0 \\
\mu(\Phi(x)-\bar{\lambda}) \rightarrow 0}} \mu \partial \Phi(x) \subset N_{S}(\bar{x}) .
\end{aligned}
$$

Let $x^{*} \in \limsup \quad \mu \partial \Phi(x)$. By definition, there exist $\left(x_{n}\right) \subset X,\left(x_{n}^{*}\right) \subset X^{*}$ $\left\{\begin{array}{l}x \rightarrow \bar{x}, \mu \geq 0 \\ \mu(\Phi(x)-\bar{\lambda}) \rightarrow 0\end{array}\right.$

and $\left(\mu_{n}\right) \subset \mathbb{R}_{+}$such that $x_{n}^{*} \in \partial \Phi\left(x_{n}\right), x_{n} \rightarrow \bar{x}, \mu_{n} x_{n}^{*} \rightarrow x^{*}$ and $\mu_{n}\left(\Phi\left(x_{n}\right)-\lambda\right)$ $\rightarrow 0$ as $n \rightarrow+\infty$. Let us fix $x \in S$. The subdifferential inequality gives

$$
\left\langle x_{n}^{*}, x-x_{n}\right\rangle \leq \Phi(x)-\Phi\left(x_{n}\right) \leq \lambda-\Phi\left(x_{n}\right) .
$$

Multiplying by $\mu_{n}$, we find $\left\langle\mu_{n} x_{n}^{*}, x-x_{n}\right\rangle \leq \mu_{n}\left(\lambda-\Phi\left(x_{n}\right)\right)$. Taking the limit as $n \rightarrow+\infty$, we obtain that $\left\langle x^{*}, x-\bar{x}\right\rangle \leq 0$ because $x_{n} \rightarrow \bar{x}, \mu_{n} x_{n}^{*} \rightarrow x^{*}$ and $\mu_{n}\left(\lambda-\Phi\left(x_{n}\right)\right) \rightarrow 0$ as $n \rightarrow+\infty$. Since this is true for every $x \in S$, we conclude that $x^{*} \in N_{S}(\bar{x})$, which shows the inclusion (3.12). By combining inclusions (3.4), (3.10) and (3.12), we obtain formulae (3.1)-(3.2).

To prove formula (3.3), let us start with the following lemma.

Lemma 3.2. Assume that there exist sequences $\left(x_{n}\right) \subset X,\left(x_{n}^{*}\right) \subset X^{*}$ and $\left(\mu_{n}\right) \subset$ $\mathbb{R}_{+}$such that $x_{n}^{*} \in \partial \Phi\left(x_{n}\right), x_{n} \rightarrow \bar{x}$ and $\mu_{n} x_{n}^{*} \rightarrow x^{*}$ as $n \rightarrow+\infty$. Then we have

$$
\lim _{n \rightarrow+\infty} \mu_{n}\left(\Phi\left(x_{n}\right)-\Phi(\bar{x})\right)=0 .
$$

Proof of Lemma 3.2. Since $x_{n}^{*} \in \partial \Phi\left(x_{n}\right)$, the subdifferential inequality gives

$$
\Phi\left(x_{n}\right)-\Phi(\bar{x}) \leq\left\langle x_{n}^{*}, x_{n}-\bar{x}\right\rangle .
$$

Multiplying by $\mu_{n}$ and taking the upper limit as $n \rightarrow+\infty$, we obtain that

$$
\limsup _{n \rightarrow+\infty} \mu_{n}\left(\Phi\left(x_{n}\right)-\Phi(\bar{x})\right) \leq 0
$$

because $x_{n} \rightarrow \bar{x}$ and $\mu_{n} x_{n}^{*} \rightarrow x^{*}$. To complete the proof, we have to show that

$$
\liminf _{n \rightarrow+\infty} \mu_{n}\left(\Phi\left(x_{n}\right)-\Phi(\bar{x})\right) \geq 0 .
$$

First assume that the sequence $\left(\mu_{n}\right)$ is bounded from above, say by $M>0$. We then have

$$
\mu_{n}\left(\Phi\left(x_{n}\right)-\Phi(\bar{x})\right) \geq-M\left(\Phi\left(x_{n}\right)-\Phi(\bar{x})\right)_{-},
$$

where $\alpha_{-}:=\max \{0,-\alpha\}$ denotes the negative part of the real $\alpha$. The lower semicontinuity of $\Phi$ implies that $\liminf _{n \rightarrow+\infty} \Phi\left(x_{n}\right) \geq \Phi(\bar{x})$, hence $\lim _{n \rightarrow+\infty}\left(\Phi\left(x_{n}\right)-\right.$ $\Phi(\bar{x}))_{-}=0$. Taking the lower limit as $n \rightarrow+\infty$ in the above inequality, we obtain (3.14).

Now assume that the sequence $\left(\mu_{n}\right)$ is not bounded from above. There exists a subsequence of $\left(\mu_{n}\right)$, still denoted by $\left(\mu_{n}\right)$ such that $\lim _{n \rightarrow+\infty} \mu_{n}=+\infty$. Observe that $x_{n}^{*}=\left(\mu_{n} x_{n}^{*}\right) / \mu_{n} \rightarrow 0$ because $\mu_{n} x_{n}^{*} \rightarrow x^{*}$ as $n \rightarrow+\infty$. Since $x_{n} \rightarrow \bar{x}$ as $n \rightarrow+\infty$ and $x_{n}^{*} \in \partial \Phi\left(x_{n}\right)$, we deduce that $0 \in \partial \Phi(\bar{x})$, due to the graph-closedness of the operator $\partial \Phi$. Therefore $\bar{x}$ is a minimizer of $\Phi$ over $X$. This implies that $\Phi\left(x_{n}\right) \geq \Phi(\bar{x})$ for every $n \in \mathbb{N}$, hence formula (3.14) is also satisfied in this case. By combining inequalities (3.13) and (3.14), we conclude that $\lim _{n \rightarrow+\infty} \mu_{n}\left(\Phi\left(x_{n}\right)-\right.$ $\Phi(\bar{x}))=0$. 
We are now able to prove formula (3.3). First assume that $\Phi(\bar{x})=\lambda$. Lemma 3.2 shows that

$$
\limsup _{x \rightarrow \bar{x}} \mathbb{R}_{+} \partial \Phi(x) \subset \limsup _{\left\{\begin{array}{l}
x \rightarrow \bar{x}, \mu \geq 0 \\
\mu(\Phi(x)-\Phi(\bar{x})) \rightarrow 0
\end{array}\right.} \mu \partial \Phi(x)
$$

and since the reverse inclusion is obviously true, both members are equal. It suffices then to use formula (3.2).

Now assume that $\Phi(\bar{x})<\lambda$. Observe that

$$
\begin{aligned}
& N_{S}(\bar{x})=\limsup _{\left\{\begin{array}{l}
x \rightarrow \bar{x}, \mu \geq 0 \\
\mu(\Phi(x)-\bar{\lambda}) \rightarrow 0
\end{array}\right.} \mu \partial \Phi(x) \quad \text { in view of formula (3.2) } \\
& \subset \quad \limsup \quad \mu \partial \Phi(x) \quad \text { from Lemma } 3.2 \\
& \left\{\begin{array}{l}
x \rightarrow \bar{x}, \mu \geq 0 \\
\mu(\Phi(x)-\bar{\lambda}) \rightarrow 0 \\
\mu(\Phi(x)-\Phi(\bar{x})) \rightarrow 0
\end{array}\right. \\
& \subset \limsup _{x \rightarrow \bar{x}, \mu \downarrow 0} \mu \partial \Phi(x) \quad \text { because } \Phi(\bar{x}) \neq \lambda \\
& =N_{\operatorname{dom} \Phi}(\bar{x}) \quad \text { from Lemma 2.6. }
\end{aligned}
$$

Since $S \subset \operatorname{dom} \Phi$, the inclusion $N_{\operatorname{dom} \Phi}(\bar{x}) \subset N_{S}(\bar{x})$ is obvious and we conclude that

$$
N_{S}(\bar{x})=\limsup _{x \rightarrow \bar{x}, \mu \downarrow 0} \mu \partial \Phi(x)=N_{\operatorname{dom} \Phi}(\bar{x}) .
$$

Alternative proof of formula (3.1). Let us define the affine continuous function $F$ : $X \rightarrow X \times \mathbb{R}$ by $F(x)=(x, \lambda)$ for every $x \in X$ and let $f: X \times \mathbb{R} \rightarrow \mathbb{R} \cup\{+\infty\}$ be the lower semicontinuous convex function given by $f=\delta_{\text {epi } \Phi}$. Observe that

$$
x \in S \Longleftrightarrow \Phi(x) \leq \lambda \Longleftrightarrow F(x) \in \operatorname{epi} \Phi \text {. }
$$

It ensues that $\delta_{S}=\delta_{\text {epi } \Phi} \circ F=f \circ F$, and hence

$$
N_{S}(\bar{x})=\partial \delta_{S}(\bar{x})=\partial(f \circ F)(\bar{x}) .
$$

By applying Corollary 2.3, we obtain that $x^{*} \in N_{S}(\bar{x})$ if and only if

$$
\left\{\begin{array}{l}
\text { there exist sequences }\left(x_{n}^{*}, t_{n}^{*}\right) \subset X^{*} \times \mathbb{R}, \quad\left(x_{n}, t_{n}\right) \subset X \times \mathbb{R} \\
\text { such that }\left(x_{n}^{*}, t_{n}^{*}\right) \in N_{\text {epi } \Phi}\left(x_{n}, t_{n}\right), \quad\left(x_{n}, t_{n}\right) \longrightarrow(\bar{x}, \lambda), \\
x_{n}^{*} \longrightarrow x^{*} \text { and }\left\langle\left(x_{n}^{*}, t_{n}^{*}\right),\left(x_{n}, t_{n}\right)-(\bar{x}, \lambda)\right\rangle \longrightarrow 0 \text { as } n \rightarrow+\infty .
\end{array}\right.
$$

Observe that $\left\langle\left(x_{n}^{*}, t_{n}^{*}\right),\left(x_{n}, t_{n}\right)-(\bar{x}, \lambda)\right\rangle=\left\langle x_{n}^{*}, x_{n}-\bar{x}\right\rangle+t_{n}^{*}\left(t_{n}-\lambda\right)$, so whenever $x_{n} \rightarrow \bar{x}$ and $x_{n}^{*} \rightarrow x^{*}$ as $n \rightarrow+\infty$, we have the equivalence

$$
\left\langle\left(x_{n}^{*}, t_{n}^{*}\right),\left(x_{n}, t_{n}\right)-(\bar{x}, \lambda)\right\rangle \underset{n \rightarrow+\infty}{\longrightarrow} 0 \Longleftrightarrow t_{n}^{*}\left(t_{n}-\lambda\right) \underset{n \rightarrow+\infty}{\longrightarrow} 0 .
$$

On the other hand, from a classical property of epigraphs, the real number $t_{n}^{*}$ satisfies $t_{n}^{*} \leq 0$. By setting $\mu_{n}=-t_{n}^{*} \geq 0$, assertion (3.15) can be reformulated as

$$
\left\{\begin{array}{l}
\text { there exist sequences }\left(x_{n}\right) \subset X,\left(x_{n}^{*}\right) \subset X^{*},\left(\mu_{n}\right) \subset \mathbb{R}_{+},\left(t_{n}\right) \subset \mathbb{R} \\
\text { such that }\left(x_{n}^{*},-\mu_{n}\right) \in N_{\text {epi }}\left(x_{n}, t_{n}\right), \quad x_{n} \longrightarrow \bar{x}, t_{n} \longrightarrow \lambda, \\
x_{n}^{*} \longrightarrow x^{*} \text { and } \mu_{n}\left(t_{n}-\lambda\right) \longrightarrow 0 \text { as } n \rightarrow+\infty .
\end{array}\right.
$$

Let us now distinguish the cases $\mu_{n}>0$ and $\mu_{n}=0$. If $\mu_{n}>0$, the inclusion $\left(x_{n}^{*},-\mu_{n}\right) \in N_{\text {epi } \Phi}\left(x_{n}, t_{n}\right)$ ensures that $t_{n}=\Phi\left(x_{n}\right)$, so the following equivalences 
hold:

$$
\begin{aligned}
\left(x_{n}^{*},-\mu_{n}\right) \in N_{\text {epi } \Phi}\left(x_{n}, t_{n}\right) & \Longleftrightarrow\left(x_{n}^{*} / \mu_{n},-1\right) \in N_{\text {epi } \Phi}\left(x_{n}, \Phi\left(x_{n}\right)\right) \\
& \Longleftrightarrow x_{n}^{*} / \mu_{n} \in \partial \Phi\left(x_{n}\right) \\
& \Longleftrightarrow x_{n}^{*} \in \mu_{n} \partial \Phi\left(x_{n}\right)=\partial\left(\mu_{n} \Phi\right)\left(x_{n}\right) .
\end{aligned}
$$

If $\mu_{n}=0$ we have the equivalences

$$
\begin{aligned}
\left(x_{n}^{*}, 0\right) \in N_{\text {epi } \Phi}\left(x_{n}, t_{n}\right) & \Longleftrightarrow x_{n}^{*} \in N_{\operatorname{dom} \Phi}\left(x_{n}\right) \\
& \Longleftrightarrow x_{n}^{*} \in \partial(0 \Phi)\left(x_{n}\right),
\end{aligned}
$$

according to the equality $0 \Phi=\delta_{\text {dom } \Phi}$. We immediately deduce from the above discussion that for every $n \in \mathbb{N}$,

$$
\mu_{n}\left(t_{n}-\lambda\right)=\mu_{n}\left(\Phi\left(x_{n}\right)-\lambda\right)
$$

and

$$
\left(x_{n}^{*},-\mu_{n}\right) \in N_{\text {epi } \Phi}\left(x_{n}, t_{n}\right) \quad \Longleftrightarrow \quad x_{n}^{*} \in \partial\left(\mu_{n} \Phi\right)\left(x_{n}\right) .
$$

By using (3.18) -(3.19), we obtain that assertion (3.17) is equivalent to

$$
\left\{\begin{array}{l}
\text { there exist sequences }\left(x_{n}\right) \subset X,\left(x_{n}^{*}\right) \subset X^{*},\left(\mu_{n}\right) \subset \mathbb{R}_{+}, \\
\text {such that } x_{n}^{*} \in \partial\left(\mu_{n} \Phi\right)\left(x_{n}\right), \quad x_{n} \longrightarrow \bar{x}, \quad x_{n}^{*} \longrightarrow x^{*} \text { and } \\
\mu_{n}\left(\Phi\left(x_{n}\right)-\lambda\right) \longrightarrow 0 \text { as } n \rightarrow+\infty,
\end{array}\right.
$$

which is in turn equivalent to $x^{*} \in \limsup \quad \partial(\mu \Phi)(x)$. We conclude that

$$
\begin{aligned}
N_{S}(\bar{x})= & \limsup _{\left\{\begin{array}{l}
x \rightarrow \bar{x}, \mu \geq 0 \\
\mu(\Phi(x)-\bar{\lambda}) \rightarrow 0
\end{array}\right.} \partial(\mu \Phi)(x) .
\end{aligned}
$$$$
\left\{\begin{array}{l}
x \rightarrow \bar{x}, \mu \geq 0 \\
\mu(\Phi(x)-\bar{\lambda}) \rightarrow 0
\end{array}\right.
$$

Remark 3.3. The set $\lim \sup \mathbb{R}_{+} \partial \Phi(x)$ arising in formula (3.3) can be described as follows:

$x^{*} \in \limsup _{x \rightarrow \bar{x}} \mathbb{R}_{+} \partial \Phi(x) \Longleftrightarrow x^{*}=0$ or $\exists x_{n} \rightarrow \bar{x}, \exists x_{n}^{*} \in \partial \Phi\left(x_{n}\right) \backslash\{0\}, \frac{x_{n}^{*}}{\left\|x_{n}^{*}\right\|} \rightarrow \frac{x^{*}}{\left\|x^{*}\right\|}$.

To prove the first implication, let us fix $x^{*} \in\left[\limsup _{x \rightarrow \bar{x}} \mathbb{R}_{+} \partial \Phi(x)\right] \backslash\{0\}$. There exist a sequence $\left(x_{n}\right)$ such that $x_{n} \rightarrow \bar{x}$ and a sequence $\left(\mu_{n}\right) \subset \mathbb{R}_{+}$along with a sequence $\left(x_{n}^{*}\right)$ such that $x_{n}^{*} \in \partial \Phi\left(x_{n}\right)$ and $\mu_{n} x_{n}^{*} \rightarrow x^{*}$. It ensues that $\mu_{n}\left\|x_{n}^{*}\right\| \rightarrow\left\|x^{*}\right\|$ as $n \rightarrow+\infty$. Since $\left\|x^{*}\right\| \neq 0$, we have $\mu_{n} \neq 0$ and $\left\|x_{n}^{*}\right\| \neq 0$ for $n$ large enough, and hence $x_{n}^{*} /\left\|x_{n}^{*}\right\| \rightarrow x^{*} /\left\|x^{*}\right\|$ as $n \rightarrow+\infty$. The reverse implication is immediate.

We now show how equality (3.3) allows us to recover classical formulae for the normals to sublevel sets under the Slater condition 3 Recall that, given a closed convex set $C \subset X$ and $x \in C$, the recession cone $C^{\infty}$ is defined by

$$
C^{\infty}=\{u \in X, \quad x+t u \in C \text { for all } t \geq 0\} .
$$

The set $C^{\infty}$ does not depend on $x \in C$ and is also given by

$$
C^{\infty}=\{u \in X, u+C \subset C\} .
$$

For more details on recession analysis, see, for example, [4, 7, 23, 31].

\footnotetext{
${ }^{3}$ See formulae (1.2)-(1.4) of the introduction.
} 
Corollary 3.4. Let $X$ be a reflexive Banach space and let $\Phi: X \rightarrow \mathbb{R} \cup\{+\infty\}$ be a lower semicontinuous convex function. Given $\bar{x} \in \operatorname{dom} \Phi$, let us consider the level set $S=[\Phi \leq \Phi(\bar{x})]$. Then we have

(i) $N_{S}(\bar{x})=\mathbb{R}_{+} \partial \Phi(\bar{x}) \cup N_{\text {dom } \Phi}(\bar{x}) \cup \limsup _{x \rightarrow \bar{x}, \mu \rightarrow+\infty} \mu \partial \Phi(x)$.

Assuming in addition the Slater condition $[\Phi<\Phi(\bar{x})] \neq \emptyset$, we obtain

(ii) $N_{S}(\bar{x})=\mathbb{R}_{+} \partial \Phi(\bar{x}) \cup N_{\text {dom } \Phi}(\bar{x})$.

(iii) If additionally $\partial \Phi(\bar{x}) \neq \emptyset$, then $N_{S}(\bar{x})=\operatorname{cl}\left(\mathbb{R}_{+} \partial \Phi(\bar{x})\right)$.

(iv) Finally, if $\bar{x} \in \operatorname{int}(\operatorname{dom} \Phi)$, we have $N_{S}(\bar{x})=\mathbb{R}_{+} \partial \Phi(\bar{x})$.

Proof. (i) In view of formula (3.3), it suffices to prove the equality

$$
\limsup _{x \rightarrow \bar{x}} \mathbb{R}_{+} \partial \Phi(x)=\mathbb{R}_{+} \partial \Phi(\bar{x}) \cup N_{\operatorname{dom} \Phi}(\bar{x}) \cup \limsup _{x \rightarrow \bar{x}, \mu \rightarrow+\infty} \mu \partial \Phi(x) .
$$

Let $x^{*} \in \limsup _{x \rightarrow \bar{x}} \mathbb{R}_{+} \partial \Phi(x)$. By definition, there exist a sequence $\left(x_{n}\right) \subset X$, a sequence $\left(x_{n}^{*}\right) \subset X^{*}$ and a sequence $\left(\mu_{n}\right) \subset \mathbb{R}_{+}$such that $x_{n} \rightarrow \bar{x}, x_{n}^{*} \rightarrow x^{*}$ as $n \rightarrow+\infty$ and $x_{n}^{*} \in \mu_{n} \partial \Phi\left(x_{n}\right)$. There exists a subsequence of $\left(\mu_{n}\right)$, still denoted by $\left(\mu_{n}\right)$, such that $\lim _{n \rightarrow+\infty} \mu_{n}=\bar{\mu} \in \mathbb{R}_{+} \cup\{+\infty\}$. If $\bar{\mu} \in \mathbb{R}_{+} \backslash\{0\}$, we deduce from the graph-closedness of the operator $\partial \Phi$ that $x^{*} / \bar{\mu} \in \partial \Phi(\bar{x})$, hence $x^{*} \in \mathbb{R}_{+} \partial \Phi(\bar{x})$. Now assume that $\lim _{n \rightarrow+\infty} \mu_{n}=0$. We then obtain that $x^{*} \in \limsup _{x \rightarrow \bar{x}, \mu \downarrow 0} \mu \partial \Phi(x)$, and therefore $x^{*} \in N_{\text {dom } \Phi}(\bar{x})$ in view of Lemma 2.6. Finally, if $\lim _{n \rightarrow+\infty} \mu_{n}=+\infty$, we find $x^{*} \in \limsup _{x \rightarrow \bar{x}, \mu \rightarrow+\infty} \mu \partial \Phi(x)$. The above arguments show that

$$
x^{*} \in \mathbb{R}_{+} \partial \Phi(\bar{x}) \cup N_{\operatorname{dom} \Phi}(\bar{x}) \cup \limsup _{x \rightarrow \bar{x}, \mu \rightarrow+\infty} \mu \partial \Phi(x),
$$

hence the first inclusion in formula (3.21) is proved. Since the reverse inclusion is obvious, the proof of $(i)$ is complete.

(ii) Let us now assume the Slater condition, thus implying that $0 \notin \partial \Phi(\bar{x})$. By using the graph-closedness of the operator $\partial \Phi$, we immediately obtain that the set $\limsup _{x \rightarrow \bar{x}} \mu \partial \Phi(x)$ is empty. Formula $(i i)$ then follows from $(i)$.

(iii) If $\partial \Phi(\bar{x}) \neq \emptyset$, then we have $N_{\operatorname{dom} \Phi}(\bar{x})=(\partial \Phi(\bar{x}))^{\infty}$; see for example 9 , Prop. 2.126]. Recalling that $\operatorname{cl}\left(\mathbb{R}_{+} C\right)=\mathbb{R}_{+} C \cup C^{\infty}$ for any nonempty closed convex set $C \subset X$ such that $0 \notin C$ and applying this fact with $C=\partial \Phi(\bar{x})$, we obtain ( $i i i)$ from $(i i)$.

(iv) If $\bar{x} \in \operatorname{int}(\operatorname{dom} \Phi)$, then we have $N_{\operatorname{dom} \Phi}(\bar{x})=\{0\}$, hence $N_{S}(\bar{x})=\mathbb{R}_{+} \partial \Phi(\bar{x})$ in view of $(i i)$.

If we assume that $\bar{x} \in \operatorname{int}(\operatorname{dom} \Phi)$, then formula (3.3) can be slightly improved, as shown by the following theorem.

Theorem 3.5. Let $X$ be a reflexive Banach space and let $\Phi: X \rightarrow \mathbb{R} \cup\{+\infty\}$ be a lower semicontinuous convex function. Given $\bar{x} \in \operatorname{int}(\operatorname{dom} \Phi)$, let us define the set $S$ by $S=[\Phi \leq \Phi(\bar{x})]$. If $\bar{x} \notin \operatorname{int}(S)$ we hav 4

$$
N_{S}(\bar{x})=\limsup _{x \rightarrow \bar{x}, x \notin S} \mathbb{R}_{+} \partial \Phi(x) .
$$

\footnotetext{
${ }^{4}$ The assumption $\bar{x} \notin \operatorname{int}(S)$ ensures that $\bar{x} \in \operatorname{cl}(X \backslash S)$, hence the upper limit arising in (3.22) is well defined. On the contrary, if $\bar{x} \in \operatorname{int}(S)$, we obviously have $N_{S}(\bar{x})=\{0\}$. It is easy to check that the assumption $\bar{x} \in \operatorname{int}(S)$ implies that $\Phi$ is constant on some neighborhood of $\bar{x}$, hence $\partial \Phi(\bar{x})=\{0\}$. We deduce that $\bar{x} \in \operatorname{argmin} \Phi$ and therefore $S=\operatorname{argmin} \Phi$ in this case.
} 
Proof. First observe that

$$
\begin{aligned}
\limsup _{x \rightarrow \bar{x}, x \notin S} \mathbb{R}_{+} \partial \Phi(x) & \subset \limsup _{x \rightarrow \bar{x}} \mathbb{R}_{+} \partial \Phi(x) \\
& =N_{S}(\bar{x}) \text { from Theorem 3.1 }
\end{aligned}
$$

Hence it suffices to prove the inclusion $N_{S}(\bar{x}) \subset \limsup _{x \rightarrow \bar{x}, x \notin S} \mathbb{R}_{+} \partial \Phi(x)$. Let us fix $x^{*} \in$ $N_{S}(\bar{x}) \backslash\{0\}$ and let us define the closed convex set $S_{n} \subset X$ by $S_{n}=[\Phi \leq \Phi(\bar{x})+1 / n]$ for every $n \geq 1$. By applying Lemma 2.5 $(i)$ with $\lambda_{n}=\Phi(\bar{x})+1 / n$, we obtain that the sequence $\left(S_{n}\right)$ Mosco-converges to the set $S=[\Phi \leq \Phi(\bar{x})]$. Theorem 2.4 (ii) then shows that there exist a sequence $\left(x_{n}\right) \subset X$ and a sequence $\left(x_{n}^{*}\right) \subset X^{*}$ such that $x_{n} \in S_{n}, x_{n}^{*} \in N_{S_{n}}\left(x_{n}\right), x_{n} \rightarrow \bar{x}$ and $x_{n}^{*} \rightarrow x^{*}$ as $n \rightarrow+\infty$. By assumption, we have $\bar{x} \in \operatorname{int}(\operatorname{dom} \Phi)$, hence there exists $n_{0} \in \mathbb{N}$ such that $x_{n} \in \operatorname{int}(\operatorname{dom} \Phi)$ for every $n \geq n_{0}$. On the other hand, since $x_{n}^{*} \rightarrow x^{*}$ as $n \rightarrow+\infty$ and $x^{*} \neq 0$, there exists $n_{1} \geq n_{0}$ such that $x_{n}^{*} \neq 0$ for every $n \geq n_{1}$. Without loss of generality, we suppose in the sequel that $n \geq n_{1}$.

Let us now prove that $\Phi\left(x_{n}\right)=\Phi(\bar{x})+1 / n$. Let us argue by contradiction and assume that $\Phi\left(x_{n}\right)<\Phi(\bar{x})+1 / n$. From a classical result, the lower semicontinuous convex function $\Phi$ is continuous on the set $\operatorname{int}(\operatorname{dom} \Phi)$, hence $\Phi$ is continuous at $x_{n}$. Since $\Phi\left(x_{n}\right)<\Phi(\bar{x})+1 / n$, we deduce that $x_{n} \in \operatorname{int}\left(S_{n}\right)$ and hence $N_{S_{n}}\left(x_{n}\right)=\{0\}$. This implies that $x_{n}^{*}=0$, which gives the contradiction. We have shown that $\Phi\left(x_{n}\right)=\Phi(\bar{x})+1 / n$, hence in particular $x_{n} \notin S$. Since $x_{n} \in \operatorname{int}(\operatorname{dom} \Phi)$ and since $\left[\Phi<\Phi\left(x_{n}\right)\right] \neq \emptyset$ because $\Phi(\bar{x})<\Phi\left(x_{n}\right)$, Corollary 3.4 (iv) can be applied with the point $x_{n}$ and the set $S_{n}=\left[\Phi \leq \Phi\left(x_{n}\right)\right]$. We deduce that $N_{S_{n}}\left(x_{n}\right)=\mathbb{R}_{+} \partial \Phi\left(x_{n}\right)$ and therefore $x_{n}^{*} \in \mathbb{R}_{+} \partial \Phi\left(x_{n}\right)$. As a conclusion, we have built a sequence $\left(x_{n}\right)$ such that $x_{n} \notin S$ for every $n \geq n_{1}$ and $x_{n} \rightarrow \bar{x}$ as $n \rightarrow+\infty$, along with a sequence $\left(x_{n}^{*}\right)$ such that $x_{n}^{*} \in \mathbb{R}_{+} \partial \Phi\left(x_{n}\right)$ for every $n \geq n_{1}$ and $x_{n}^{*} \rightarrow x^{*}$ as $n \rightarrow+\infty$. This shows that $x^{*} \in \limsup \mathbb{R}_{+} \partial \Phi(x)$, which proves the inclusion $N_{S}(\bar{x}) \backslash\{0\} \subset$ $\limsup \mathbb{R}_{+} \partial \Phi(x)$. It remains to establish that $0 \in \lim \sup \mathbb{R}_{+} \partial \Phi(x)$. Since $x \rightarrow \bar{x}, x \notin S$ $\bar{x} \notin \operatorname{int}(S)$ by assumption, there exists a sequence $\left(x_{n}\right) \subset X$ such that $x_{n} \notin S$ and $x_{n} \rightarrow \bar{x}$ as $n \rightarrow+\infty$. Recalling that $\bar{x} \in \operatorname{int}(\operatorname{dom} \Phi)$, we have $x_{n} \in \operatorname{int}(\operatorname{dom} \Phi)$ for $n$ large enough, thus $\Phi$ is continuous at $x_{n}$. It ensues that $\partial \Phi\left(x_{n}\right) \neq \emptyset$, hence $0 \in \mathbb{R}_{+} \partial \Phi\left(x_{n}\right)$ for $n$ large enough and we conclude that $0 \in \limsup _{x \rightarrow \bar{x}, x \notin S} \mathbb{R}_{+} \partial \Phi(x)$.

Remark 3.6. If $\bar{x} \notin \operatorname{int}(\operatorname{dom} \Phi)$, formula (3.22) of the above theorem may fail. Take for example $X=\mathbb{R}, \Phi=\delta_{\mathbb{R}_{+}}$and $\bar{x}=0$. We have $N_{S}(\bar{x})=N_{\mathbb{R}_{+}}(0)=\mathbb{R}_{-}$, while

$$
\limsup _{x \rightarrow \bar{x}, x \notin S} \mathbb{R}_{+} \partial \Phi(x)=\limsup _{x \rightarrow 0, x<0} \mathbb{R}_{+} \partial \Phi(x)=\emptyset .
$$

\section{Extension to nonReflexive Banach spaces}

This section is concerned with the normal cone to convex sublevel set of nonreflexive Banach space. In such a framework we obtain the following result similar to Theorem 3.1, but here the upper limit has to be taken with respect to the weak-star topology of $X^{*}$ and an additional condition involving the bracket $\langle\cdot, x-\bar{x}\rangle$ needs to be required. 
Theorem 4.1. Let $X$ be a Banach space and let $\Phi: X \rightarrow \mathbb{R} \cup\{+\infty\}$ be a lower semicontinuous convex function. For $\lambda \in \mathbb{R}$, let $S=[\Phi \leq \lambda]$ and let $\bar{x} \in S$. Then

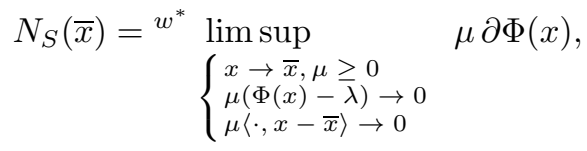

where the second member stands for the set of weak-star limits ${ }^{w^{*}} \lim \mu_{i} u_{i}^{*}$ such that $\mu_{i} \geq 0, u_{i}^{*} \in \partial \Phi\left(x_{i}\right)$, the net $\left(x_{i}\right)_{i}$ strongly converges to $\bar{x}, \mu_{i}\left(\Phi\left(x_{i}\right)-\lambda\right) \rightarrow 0$ and $\mu_{i}\left\langle u_{i}^{*}, x_{i}-\bar{x}\right\rangle \rightarrow 0$.

Further, if $\Phi(\bar{x})<\lambda$ one also has

$$
N_{S}(\bar{x})=w^{*} \limsup _{\left\{\begin{array}{l}
x \rightarrow \bar{x}, \mu \downarrow 0 \\
\mu\langle\cdot, x-\bar{x}\rangle \rightarrow 0
\end{array}\right.} \mu \partial \Phi(x)=N_{\operatorname{dom} \Phi}(\bar{x}) .
$$

Proof. First we note that in the proof of Theorem 3.1, according to Theorem 2.2 $(c)$, $\left(x_{n}\right)_{n},\left(y_{n}\right)_{n},\left(x_{n}^{*}\right)_{n},\left(y_{n}^{*}\right)_{n}$, and $\left(e_{n}^{*}\right)$ have to be taken as nets instead of sequences, the strong convergence $x_{n}^{*} \rightarrow x^{*}$ has to be replaced by the weak-star convergence $x_{n}^{*} \stackrel{w^{*}}{\rightarrow} x^{*}$, and we have in addition $\left\langle x_{n}^{*}, x_{n}-\bar{x}\right\rangle \rightarrow 0$. With those elements at hand, one can show with arguments in the line of Theorem 3.1 that

$$
\begin{aligned}
& N_{S}(\bar{x}) \subset w^{w^{*}} \limsup \quad \partial(\mu \Phi)(x), \\
& \left\{\begin{array}{l}
x \rightarrow \bar{x}, \mu \geq 0 \\
\mu(\Phi(x)-\bar{\lambda}) \rightarrow 0 \\
\langle\cdot, x-\bar{x}\rangle \rightarrow 0
\end{array}\right.
\end{aligned}
$$

where the second member denotes the set of weak-star limits of nets $\left(z_{n}^{*}\right)_{n}$ for which there are a net $\left(x_{n}\right)_{n}$ converging to $\bar{x}$ and a net $\left(\mu_{n}\right)_{n}$ in $\mathbb{R}_{+}$such that $z_{n}^{*} \in \partial\left(\mu_{n} \Phi\right)\left(x_{n}\right), \mu_{n}\left(\Phi\left(x_{n}\right)-\lambda\right) \rightarrow 0$ and $\left\langle z_{n}^{*}, x_{n}-\bar{x}\right\rangle \rightarrow 0$. Let us show that the latter second member is included in the same upper limit but with $\mu \partial \Phi(x)$ in place of $\partial(\mu \Phi)(x)$. Let $x^{*}$ be in the upper limit with $\partial(\mu \Phi)(x)$. By definition, there exist nets $x_{n} \rightarrow \bar{x}, x_{n}^{*} \stackrel{w^{*}}{\rightarrow} x^{*},\left(\mu_{n}\right) \subset \mathbb{R}_{+}$satisfying $x_{n}^{*} \in \partial\left(\mu_{n} \Phi\right)\left(x_{n}\right), \mu_{n}\left(\Phi\left(x_{n}\right)-\lambda\right) \rightarrow 0$ and $\left\langle x_{n}^{*}, x_{n}-\bar{x}\right\rangle \rightarrow 0$. First suppose that there exists a subnet of $\left(\mu_{n}\right)$, still denoted by $\left(\mu_{n}\right)$, such that $\mu_{n}>0$ for every $n$. We then have $x_{n}^{*} \in \mu_{n} \partial \Phi\left(x_{n}\right)$ and hence

$$
\begin{aligned}
& x^{*} \in w^{w^{*}} \limsup \quad \mu \partial \Phi(x) . \\
& \left\{\begin{array}{l}
x \rightarrow \bar{x}, \mu \geq 0 \\
\mu(\Phi(x)-\bar{\lambda}) \rightarrow 0 \\
\mu\langle\cdot, x-\bar{x}\rangle \rightarrow 0
\end{array}\right.
\end{aligned}
$$

Now suppose, for some element $n_{0}$ of the set of indices, that $\mu_{n}=0$ for all $n \succeq n_{0}$. Recalling that $0 \Phi=\delta_{\operatorname{dom} \Phi}$, we obtain $x_{n}^{*} \in N_{\operatorname{dom} \Phi}\left(x_{n}\right)$ for $n$ large enough. Writing, for every $x \in \operatorname{dom} \Phi$,

$$
\left\langle x_{n}^{*}, x-\bar{x}\right\rangle=\left\langle x_{n}^{*}, x-x_{n}\right\rangle+\left\langle x_{n}^{*}, x_{n}-\bar{x}\right\rangle \leq\left\langle x_{n}^{*}, x_{n}-\bar{x}\right\rangle,
$$

and taking the convergence $\left\langle x_{n}^{*}, x_{n}-\bar{x}\right\rangle \rightarrow 0$ into account, we see at the limit on $n$ that $x^{*} \in N_{\operatorname{dom} \Phi}(\bar{x})$. In view of Lemma 2.6, we have $N_{\operatorname{dom} \Phi}(\bar{x})=\limsup _{\substack{\Phi \\ \Phi}, \mu \downarrow 0} \mu \partial \Phi(x)$, hence

$$
\begin{aligned}
x^{*} \in \limsup _{\{\rightarrow \bar{x}, \mu \downarrow 0} \mu \partial \Phi(x) \subset{ }^{w^{*}} & \limsup \\
\{(x) \rightarrow \Phi(\bar{x}) & \left\{\begin{array}{l}
x \rightarrow \bar{x}, \mu \geq 0 \\
\mu(\Phi(x)-\lambda) \rightarrow 0 \\
\mu(\cdot, x-\bar{x}\rangle \rightarrow 0
\end{array}\right.
\end{aligned}
$$

Therefore the inclusion (4.2) is satisfied in both cases, which proves the desired inclusion. 
Now let us show the inclusion

$$
\begin{aligned}
& w^{*} \limsup \quad \mu \partial \Phi(x) \subset N_{S}(\bar{x}) . \\
& \left\{\begin{array}{l}
x \rightarrow \bar{x}, \mu \geq 0 \\
\mu(\Phi(x)-\lambda) \rightarrow 0 \\
\mu\langle\cdot, x-\bar{x}\rangle \rightarrow 0
\end{array}\right.
\end{aligned}
$$

Fix $x^{*}$ in the first member. By definition, there are nets $\left(x_{n}\right) \subset X,\left(x_{n}^{*}\right) \subset X^{*}$ and $\left(\mu_{n}\right) \subset \mathbb{R}_{+}$such that $x_{n}^{*} \in \partial \Phi\left(x_{n}\right), x_{n} \rightarrow \bar{x}, \mu_{n} x_{n}^{*} \stackrel{w^{*}}{\rightarrow} x^{*}, \mu_{n}\left(\Phi\left(x_{n}\right)-\lambda\right) \rightarrow 0$, and $\mu_{n}\left\langle x_{n}^{*}, x_{n}-\bar{x}\right\rangle \rightarrow 0$. For any fixed element $x \in S$, the subdifferential inequality gives

$$
\left\langle\mu_{n} x_{n}^{*}, x-x_{n}\right\rangle \leq \mu_{n}\left(\Phi(x)-\Phi\left(x_{n}\right)\right) \leq \mu_{n}\left(\lambda-\Phi\left(x_{n}\right)\right) ;
$$

thus $\left\langle x^{*}, x-\bar{x}\right\rangle \leq 0$ because $\mu_{n} x_{n}^{*} \stackrel{w^{*}}{\rightarrow} x^{*}, \mu_{n}\left\langle x_{n}^{*}, x_{n}-\bar{x}\right\rangle \rightarrow 0$, and $\mu_{n}\left(\lambda-\Phi\left(x_{n}\right)\right) \rightarrow 0$. We deduce that $x^{*} \in N_{S}(\bar{x})$, which shows inclusion (4.3) and finishes the proof of the first equality of the theorem.

Finally, assume that $\Phi(\bar{x})<\lambda$. Under this additional hypothesis, for the nets at the beginning of the proof of the theorem, we obtain as in (3.8) that $y_{n}^{*} \downarrow 0$ or equivalently $\mu_{n} \downarrow 0$, since $\mu_{n}=y_{n}^{*}$. The inclusion

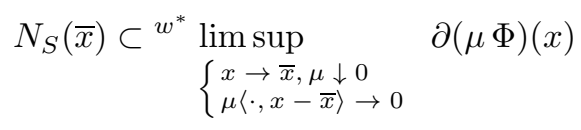

follows immediately, and by arguing as above we deduce that

$$
\begin{gathered}
N_{S}(\bar{x}) \subset w^{*} \limsup _{\left\{\begin{array}{l}
x \rightarrow \bar{x}, \mu \downarrow 0 \\
\mu\langle\cdot, x-\bar{x}\rangle \rightarrow 0
\end{array}\right.} \mu \partial \Phi(x) .
\end{gathered}
$$

Let us now show the inclusion

$$
\begin{aligned}
& w^{*} \limsup \quad \mu \partial \Phi(x) \subset N_{\operatorname{dom} \Phi}(\bar{x}) . \\
& \left\{\begin{array}{l}
x \rightarrow \bar{x}, \mu \downarrow 0 \\
\mu\langle\cdot, x-\bar{x}\rangle \rightarrow 0
\end{array}\right.
\end{aligned}
$$

Fix any $x^{*}$ in the left member. By definition there exist nets $\left(\mu_{n}\right)_{n}$ in $\mathbb{R}_{+}$with $\mu_{n} \rightarrow 0,\left(x_{n}\right)_{n}$ in $X$ converging strongly to $\bar{x},\left(u_{n}^{*}\right)_{n}$ in $X^{*}$ with $u_{n}^{*} \in \partial \Phi\left(x_{n}\right)$ such that $\mu_{n} u_{n}^{*} \stackrel{w^{*}}{\rightarrow} x^{*}$ and $\mu_{n}\left\langle u_{n}^{*}, x_{n}-\bar{x}\right\rangle \rightarrow 0$. Consider any $x \in \operatorname{dom} \Phi$ and any real $\varepsilon>0$. From the lower semicontinuity of $\Phi$, choose some index element $n_{0}$ such that for any $n \succeq n_{0}$ we have $\Phi\left(x_{n}\right) \geq \Phi(\bar{x})-\varepsilon$, where $\preceq$ denotes the directed preorder of the set of elements $n$. Then, for every $n \succeq n_{0}$ we have

$$
\left\langle u_{n}^{*}, x-x_{n}\right\rangle \leq \Phi(x)-\Phi\left(x_{n}\right) \leq \Phi(x)-\Phi(\bar{x})+\varepsilon,
$$

hence

$$
\begin{aligned}
\left\langle\mu_{n} u_{n}^{*}, x-\bar{x}\right\rangle & =\left\langle\mu_{n} u_{n}^{*}, x-x_{n}\right\rangle+\left\langle\mu_{n} u_{n}^{*}, x_{n}-\bar{x}\right\rangle \\
& \leq \mu_{n}(\Phi(x)-\Phi(\bar{x})+\varepsilon)+\mu_{n}\left\langle u_{n}^{*}, x_{n}-\bar{x}\right\rangle,
\end{aligned}
$$

so passing to the limit gives $\left\langle x^{*}, x-\bar{x}\right\rangle \leq 0$. Since this is true for every $x \in \operatorname{dom} \Phi$, we obtain $x^{*} \in N_{\operatorname{dom} \Phi}(\bar{x})$, which confirms the inclusion (4.5). Finally, since $\bar{x} \in$ $S \subset \operatorname{dom} \Phi$, it is easily seen that

$$
N_{\text {dom } \Phi}(\bar{x}) \subset N_{S}(\bar{x}) .
$$

By combining the inclusions (4.4), (4.5) and (4.6), we find the equalities in (4.1). The proof is then complete. 


\section{Extension to the intersection of Finitely MANy SUbleVEl SETS}

In this section, we consider $k$ functions $\Phi_{1}, \ldots, \Phi_{k}: X \rightarrow \mathbb{R} \cup\{+\infty\}$, which are assumed to be lower semicontinuous and convex. For $\lambda_{1}, \ldots, \lambda_{k} \in \mathbb{R}$, let us define the set $S$ by

$$
\begin{aligned}
S & =\left\{x \in X, \Phi_{1}(x) \leq \lambda_{1}, \ldots, \Phi_{k}(x) \leq \lambda_{k}\right\} \\
& =\bigcap_{i=1}^{k}\left[\Phi_{i} \leq \lambda_{i}\right] .
\end{aligned}
$$

Given $\bar{x} \in S$, our aim is to give a sequential formula for the normal cone $N_{S}(\bar{x})$, without resorting to any qualification condition. The key ingredients in that direction are Theorems 2.1 and 2.2. We focus our attention on the case where the Banach space $X$ is reflexive; of course, adaptations to the case of a nonreflexive Banach space can be realized as in Section 4. First a suitable application of Theorem 2.2 leads to the following result.

Theorem 5.1. Let $X$ be a reflexive Banach space and let $\Phi_{1}, \ldots, \Phi_{k}: X \rightarrow \mathbb{R} \cup$ $\{+\infty\}$ be lower semicontinuous convex functions. Given $\lambda_{1}, \ldots, \lambda_{k} \in \mathbb{R}$, let us define the set $S$ by $S=\bigcap_{i=1}^{k}\left[\Phi_{i} \leq \lambda_{i}\right]$. If $\bar{x} \in S$, we have

$$
N_{S}(\bar{x})=\limsup _{\left\{\begin{array}{l}
x \rightarrow \bar{x}, \mu_{i} \geq 0 \\
\sum_{i=1}^{k} \mu_{i}\left(\Phi_{i}(x)-\lambda_{i}\right) \rightarrow 0
\end{array}\right.} \partial\left[\sum_{i=1}^{k} \mu_{i} \Phi_{i}\right](x) .
$$

Proof. Let us start with the inclusion $\subset$. Analogously to the proof of Theorem 3.1 . define the function $F: X \rightarrow \mathbb{R}^{k} \cup\{+\infty\}$ by

$$
F(x)= \begin{cases}\left(\Phi_{1}(x)-\lambda_{1}, \ldots, \Phi_{k}(x)-\lambda_{k}\right) & \text { if } x \in \bigcap_{i=1}^{k} \operatorname{dom} \Phi_{i}, \\ +\infty & \text { otherwise. }\end{cases}
$$

The space $Y=\mathbb{R}^{k}$ is endowed with the preorder $\preccurlyeq$ via the convex cone $Y_{+}=\left(\mathbb{R}_{+}\right)^{k}$, that is, $y_{1} \preccurlyeq y_{2} \Leftrightarrow y_{2}-y_{1} \in\left(\mathbb{R}_{+}\right)^{k}$. The abstract maximal element $\{+\infty\}$ is adjoined to $\mathbb{R}^{k}$. The convexity of each function $\Phi_{i}$ implies the convexity of $F$ in the vector sense. The closedness of epi $F$ is easily obtained from the closedness of each epigraph epi $\Phi_{i}, i \in\{1, \ldots, k\}$. Now observe that $S=\left\{x \in X, F(x) \in\left(\mathbb{R}_{-}\right)^{k}\right\}$ and hence $\delta_{S}=\delta_{\left(\mathbb{R}_{-}\right)^{k}} \circ F$. This implies that

$$
N_{S}(\bar{x})=\partial \delta_{S}(\bar{x})=\partial\left(\delta_{\left(\mathbb{R}_{-}\right)^{k}} \circ F\right)(\bar{x}) .
$$

The function $\delta_{\left(\mathbb{R}_{-}\right)^{k}}$ is lower semicontinuous convex and nondecreasing with respect to the preorder $\preccurlyeq$. Let us fix $x^{*} \in N_{S}(\bar{x})$ and apply Theorem $2.2(a)$ with $Y=\mathbb{R}^{k}$, $Y_{+}=\left(\mathbb{R}_{+}\right)^{k}, f=\delta_{\left(\mathbb{R}_{-}\right)^{k}}$ and the function $F$ defined above. We obtain the existence of $\left(x_{n}\right) \subset X,\left(y_{n}\right) \subset \mathbb{R}^{k},\left(x_{n}^{*}\right) \subset X^{*},\left(e_{n}^{*}\right) \subset \mathbb{R}^{k}$ and $\left(y_{n}^{*}\right) \subset\left(\mathbb{R}_{+}\right)^{k}$ such that $x_{n} \rightarrow \bar{x}$, $y_{n} \rightarrow F(\bar{x}), x_{n}^{*} \rightarrow x^{*}, e_{n}^{*} \rightarrow 0$ and

$$
y_{n}^{*}+e_{n}^{*} \in N_{\left(\mathbb{R}_{-}\right)^{k}}\left(y_{n}\right), x_{n}^{*} \in \partial\left(y_{n}^{*} \circ F\right)\left(x_{n}\right) \text { and }\left\langle y_{n}^{*}, F\left(x_{n}\right)-F(\bar{x})\right\rangle \rightarrow 0 .
$$

Let us denote respectively by $\left(y_{1, n}, \ldots, y_{k, n}\right),\left(y_{1, n}^{*}, \ldots, y_{k, n}^{*}\right)$ and $\left(e_{1, n}^{*}, \ldots, e_{k, n}^{*}\right)$ the coordinates of the vectors $y_{n}, y_{n}^{*}$ and $e_{n}^{*}$ in the canonical basis of $\mathbb{R}^{k}$. Since $y_{n}^{*} \circ F=\sum_{i=1}^{k} y_{i, n}^{*}\left(\Phi_{i}-\lambda_{i}\right)$ according to (2.1), we obtain

$$
x_{n}^{*} \in \partial\left[\sum_{i=1}^{k} y_{i, n}^{*} \Phi_{i}\right]\left(x_{n}\right)
$$


and

$$
\sum_{i=1}^{k} y_{i, n}^{*}\left(\Phi_{i}\left(x_{n}\right)-\Phi_{i}(\bar{x})\right) \rightarrow 0 \quad \text { as } n \rightarrow+\infty .
$$

Let us denote by $I(\bar{x})$ the set of active indices: $I(\bar{x})=\left\{i \in\{1, \ldots, k\}, \Phi_{i}(\bar{x})=\lambda_{i}\right\}$. Let us fix $i \notin I(\bar{x})$. Since $y_{i, n} \rightarrow \Phi_{i}(\bar{x})-\lambda_{i}$ as $n \rightarrow+\infty$ and since $\Phi_{i}(\bar{x})<\lambda_{i}$, we have $y_{i, n}<0$ for $n$ large enough. It ensues that $N_{\mathbb{R}_{-}}\left(y_{i, n}\right)=\{0\}$, and formula $y_{i, n}^{*}+e_{i, n}^{*} \in N_{\mathbb{R}_{-}}\left(y_{n}\right)$ then implies that $y_{i, n}^{*}=-e_{i, n}^{*} \rightarrow 0$ as $n \rightarrow+\infty$. Hence we have proved that

$$
\forall i \notin I(\bar{x}), \quad y_{i, n}^{*} \rightarrow 0 \text { as } n \rightarrow+\infty .
$$

Now observe that

$$
\begin{aligned}
\sum_{i=1}^{k} y_{i, n}^{*}\left(\Phi_{i}\left(x_{n}\right)-\lambda_{i}\right) & =\sum_{i=1}^{k} y_{i, n}^{*}\left(\Phi_{i}\left(x_{n}\right)-\Phi_{i}(\bar{x})\right)+\sum_{i=1}^{k} y_{i, n}^{*}\left(\Phi_{i}(\bar{x})-\lambda_{i}\right) \\
& =\sum_{i=1}^{k} y_{i, n}^{*}\left(\Phi_{i}\left(x_{n}\right)-\Phi_{i}(\bar{x})\right)+\sum_{i \notin I(\bar{x})} y_{i, n}^{*}\left(\Phi_{i}(\bar{x})-\lambda_{i}\right) .
\end{aligned}
$$

In view of (5.3), (5.4) and (5.5), we immediately deduce that

$$
\sum_{i=1}^{k} y_{i, n}^{*}\left(\Phi_{i}\left(x_{n}\right)-\lambda_{i}\right) \rightarrow 0 \text { as } n \rightarrow+\infty .
$$

Finally, we have built sequences $x_{n} \rightarrow \bar{x}, x_{n}^{*} \rightarrow x^{*},\left(y_{i, n}^{*}\right) \subset \mathbb{R}_{+}$satisfying (5.2) and (5.6), which clearly shows that

$$
x^{*} \in \limsup _{\left\{\begin{array}{l}
x \rightarrow \bar{x}, \mu_{i} \geq 0 \\
\sum_{i=1}^{k} \mu_{i}\left(\Phi_{i}(x)-\lambda_{i}\right) \rightarrow 0
\end{array}\right.} \partial\left[\sum_{i=1}^{k} \mu_{i} \Phi_{i}\right](x) .
$$

Conversely, assume that $x^{*}$ satisfies (5.7) and let us prove that $x^{*} \in N_{S}(\bar{x})$. By definition, there exist sequences $x_{n} \rightarrow \bar{x}, x_{n}^{*} \rightarrow x^{*},\left(\mu_{i, n}\right) \subset \mathbb{R}_{+}, i \in\{1, \ldots, k\}$, verifying

$$
x_{n}^{*} \in \partial\left[\sum_{i=1}^{k} \mu_{i, n} \Phi_{i}\right]\left(x_{n}\right) \quad \text { and } \quad \sum_{i=1}^{k} \mu_{i, n}\left(\Phi_{i}\left(x_{n}\right)-\lambda_{i}\right) \rightarrow 0 \text { as } n \rightarrow+\infty .
$$

Let us fix $x \in S$. By using the first relation above and the convexity of the function $\sum_{i=1}^{k} \mu_{i, n} \Phi_{i}$, we find

$$
\begin{aligned}
\left\langle x_{n}^{*}, x-x_{n}\right\rangle & \leq \sum_{i=1}^{k} \mu_{i, n}\left(\Phi_{i}(x)-\Phi_{i}\left(x_{n}\right)\right) \\
& \leq \sum_{i=1}^{k} \mu_{i, n}\left(\lambda_{i}-\Phi_{i}\left(x_{n}\right)\right) \quad \text { since } x \in S .
\end{aligned}
$$

Recalling that $x_{n} \rightarrow \bar{x}, x_{n}^{*} \rightarrow x^{*}$ and $\sum_{i=1}^{k} \mu_{i, n}\left(\lambda_{i}-\Phi_{i}\left(x_{n}\right)\right) \rightarrow 0$ as $n \rightarrow+\infty$, and taking the limit as $n \rightarrow+\infty$ in the above inequality, we obtain that $\left\langle x^{*}, x-\bar{x}\right\rangle \leq 0$. Since this is true for every $x \in S$, we conclude that $x^{*} \in N_{S}(\bar{x})$.

Remark 5.2. Condition $\sum_{i=1}^{k} \mu_{i}\left(\Phi_{i}(x)-\lambda_{i}\right) \rightarrow 0$ can be interpreted as a relaxed complementary slackness condition. 
Theorem 5.3. Let $X$ be a reflexive Banach space and let $\Phi_{1}, \ldots, \Phi_{k}: X \rightarrow \mathbb{R} \cup$ $\{+\infty\}$ be lower semicontinuous convex functions. Given $\lambda_{1}, \ldots, \lambda_{k} \in \mathbb{R}$, let us define the set $S$ by $S=\bigcap_{i=1}^{k}\left[\Phi_{i} \leq \lambda_{i}\right]$. If $\bar{x} \in S$, we have

$$
N_{S}(\bar{x}) \subset \limsup _{\left\{\begin{array}{l}
x \rightarrow \bar{x}, \mu_{i} \geq 0 \\
\mu_{i} \rightarrow 0 \text { if } i \notin I(\bar{x})
\end{array}\right.} \partial\left[\sum_{i=1}^{k} \mu_{i} \Phi_{i}\right](x),
$$

with $I(\bar{x})=\left\{i \in\{1, \ldots, k\}, \Phi_{i}(\bar{x})=\lambda_{i}\right\}$. If moreover the following Slater qualification condition is satisfied,

$$
\bigcap_{i=1}^{k}\left[\Phi_{i}<\lambda_{i}\right] \neq \emptyset
$$

then the above inclusion holds as an equality.

Proof. The inclusion (5.8) is obtained as a by-product of the proof of Theorem 5.1 . see formula (5.4) in particular. The proof of the reverse inclusion is based on the following lemma.

Lemma 5.4. Assume that there exist sequences $\left(x_{n}\right) \subset X,\left(x_{n}^{*}\right) \subset X^{*}$ and $\left(\mu_{i, n}\right) \subset$ $\mathbb{R}_{+}$for $i=1, \ldots, k$ such that $x_{n} \rightarrow \bar{x}, x_{n}^{*} \rightarrow x^{*}, \mu_{i, n} \rightarrow 0$ if $i \notin I(\bar{x})$ and $x_{n}^{*} \in \partial\left[\sum_{i=1}^{k} \mu_{i, n} \Phi_{i}\right]\left(x_{n}\right)$. Assume moreover that the Slater condition (5.9) holds. Then the following properties are satisfied:

(i) There exists $M>0$ such that $\sum_{i=1}^{k} \mu_{i, n} \leq M$ for every $n \in \mathbb{N}$.

(ii) $\lim _{n \rightarrow+\infty} \sum_{i=1}^{k} \mu_{i, n}\left(\Phi_{i}\left(x_{n}\right)-\Phi_{i}(\bar{x})\right)=0$.

Proof of Lemma 5.4. (i) Let us argue by contradiction and assume that the sequence $\left(M_{n}\right)$ defined by $M_{n}=\sum_{i=1}^{k} \mu_{i, n}$ is not bounded. There exists a subsequence of $\left(M_{n}\right)$, still denoted by $\left(M_{n}\right)$, such that $\lim _{n \rightarrow+\infty} M_{n}=+\infty$. For every $i \in\{1, \ldots, k\}$, let us define the sequence $\left(\nu_{i, n}\right)$ by $\nu_{i, n}=\mu_{i, n} / M_{n}$. Each sequence $\left(\nu_{i, n}\right)$ satisfies $\nu_{i, n} \in[0,1]$ for every $n \in \mathbb{N}$. Hence we can extract a subsequence of $\left(\nu_{i, n}\right)$, still denoted by $\left(\nu_{i, n}\right)$, such that $\lim _{n \rightarrow+\infty} \nu_{i, n}=\bar{\nu}_{i} \in[0,1]$, for every $i \in\{1, \ldots, k\}$. The real numbers $\bar{\nu}_{i}$ satisfy $\sum_{i=1}^{k} \bar{\nu}_{i}=1$ and $\bar{\nu}_{i}=0$ if $i \notin I(\bar{x})$ because $\mu_{i, n} \rightarrow 0$ in this case. Let us now fix $x \in \bigcap_{i=1}^{k}\left[\Phi_{i}<\lambda_{i}\right]$, which is nonempty by assumption. Observing that $x_{n}^{*} / M_{n} \in \partial\left[\sum_{i=1}^{k} \nu_{i, n} \Phi_{i}\right]\left(x_{n}\right)$, the subdifferential inequality yields

$$
\sum_{i=1}^{k} \nu_{i, n} \Phi_{i}(x) \geq \sum_{i=1}^{k} \nu_{i, n} \Phi_{i}\left(x_{n}\right)+\left\langle x_{n}^{*} / M_{n}, x-x_{n}\right\rangle .
$$

Since $x_{n} \rightarrow \bar{x}, x_{n}^{*} \rightarrow x^{*}$ and $M_{n} \rightarrow+\infty$ as $n \rightarrow+\infty$, we immediately obtain

$$
\lim _{n \rightarrow+\infty}\left\langle x_{n}^{*} / M_{n}, x-x_{n}\right\rangle=0 .
$$

On the other hand, we have

$$
\lim _{n \rightarrow+\infty} \sum_{i=1}^{k} \nu_{i, n} \Phi_{i}(x)=\sum_{i=1}^{k} \bar{\nu}_{i} \Phi_{i}(x),
$$


because $\lim _{n \rightarrow+\infty} \nu_{i, n}=\bar{\nu}_{i}$. Finally, the lower semicontinuity of each function $\Phi_{i}$ implies that

$$
\liminf _{n \rightarrow+\infty} \nu_{i, n} \Phi_{i}\left(x_{n}\right) \geq \bar{\nu}_{i} \Phi_{i}(\bar{x})
$$

hence

$$
\liminf _{n \rightarrow+\infty} \sum_{i=1}^{k} \nu_{i, n} \Phi_{i}\left(x_{n}\right) \geq \sum_{i=1}^{k} \liminf _{n \rightarrow+\infty} \nu_{i, n} \Phi_{i}\left(x_{n}\right) \geq \sum_{i=1}^{k} \bar{\nu}_{i} \Phi_{i}(\bar{x}) .
$$

By combining (5.10), (5.11), (5.12) and (5.13), we find $\sum_{i=1}^{k} \bar{\nu}_{i} \Phi_{i}(x) \geq \sum_{i=1}^{k} \bar{\nu}_{i} \Phi_{i}(\bar{x})$. Recalling that $\bar{\nu}_{i}=0$ if $i \notin I(\bar{x})$, we infer that

$$
\sum_{i \in I(\bar{x})} \bar{\nu}_{i} \Phi_{i}(x) \geq \sum_{i \in I(\bar{x})} \bar{\nu}_{i} \Phi_{i}(\bar{x})=\sum_{i \in I(\bar{x})} \bar{\nu}_{i} \lambda_{i}
$$

On the other hand, since $x \in \bigcap_{i=1}^{k}\left[\Phi_{i}<\lambda_{i}\right]$ and since $\bar{\nu}_{i}>0$ for at least one $i \in I(\bar{x})$, we have

$$
\sum_{i \in I(\bar{x})} \bar{\nu}_{i} \Phi_{i}(x)<\sum_{i \in I(\bar{x})} \bar{\nu}_{i} \lambda_{i}
$$

which yields a contradiction with (5.14).

(ii) From $(i)$, there exists $M>0$ such that $\sum_{i=1}^{k} \mu_{i, n} \leq M$ for every $n \in \mathbb{N}$. We then have

$$
\mu_{i, n}\left(\Phi_{i}\left(x_{n}\right)-\Phi_{i}(\bar{x})\right) \geq-M\left(\Phi_{i}\left(x_{n}\right)-\Phi_{i}(\bar{x})\right)_{-} .
$$

The lower semicontinuity of $\Phi_{i}$ implies that $\liminf _{n \rightarrow+\infty} \Phi_{i}\left(x_{n}\right) \geq \Phi_{i}(\bar{x})$, hence $\lim _{n \rightarrow+\infty}\left(\Phi_{i}\left(x_{n}\right)-\Phi_{i}(\bar{x})\right)_{-}=0$. Taking the lower limit as $n \rightarrow+\infty$ in the above inequality, we obtain $\liminf \inf _{n \rightarrow+\infty} \mu_{i, n}\left(\Phi_{i}\left(x_{n}\right)-\Phi_{i}(\bar{x})\right) \geq 0$. It ensues that

$$
\liminf _{n \rightarrow+\infty} \sum_{i=1}^{k}\left[\mu_{i, n}\left(\Phi_{i}\left(x_{n}\right)-\Phi_{i}(\bar{x})\right)\right] \geq \sum_{i=1}^{k} \liminf _{n \rightarrow+\infty}\left[\mu_{i, n}\left(\Phi_{i}\left(x_{n}\right)-\Phi_{i}(\bar{x})\right)\right] \geq 0
$$

By using the assumption $x_{n}^{*} \in \partial\left[\sum_{i=1}^{k} \mu_{i, n} \Phi_{i}\right]\left(x_{n}\right)$, the subdifferential inequality gives

$$
\sum_{i=1}^{k}\left[\mu_{i, n}\left(\Phi_{i}\left(x_{n}\right)-\Phi_{i}(\bar{x})\right)\right] \leq\left\langle x_{n}^{*}, x_{n}-\bar{x}\right\rangle .
$$

Since $\lim _{n \rightarrow+\infty}\left\langle x_{n}^{*}, x_{n}-\bar{x}\right\rangle=0$, we deduce that

$$
\limsup _{n \rightarrow+\infty} \sum_{i=1}^{k}\left[\mu_{i, n}\left(\Phi_{i}\left(x_{n}\right)-\Phi_{i}(\bar{x})\right)\right] \leq 0 .
$$

By combining inequalities (5.15) and (5.16), we conclude that

$$
\lim _{n \rightarrow+\infty} \sum_{i=1}^{k}\left[\mu_{i, n}\left(\Phi_{i}\left(x_{n}\right)-\Phi_{i}(\bar{x})\right)\right]=0 .
$$


Let us come back to the proof of Theorem 5.3. Lemma 5.4 shows that

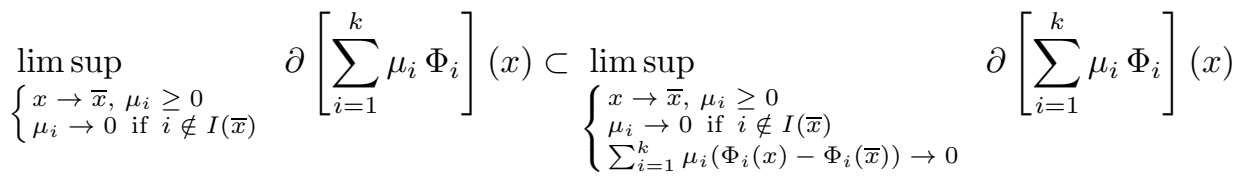

$$
\begin{aligned}
& \subset \limsup _{\left\{\begin{array}{l}
x \rightarrow \bar{x}, \mu_{i} \geq 0 \\
\sum_{i=1}^{k} \mu_{i}\left(\Phi_{i}(x)-\lambda_{i}\right) \rightarrow 0
\end{array}\right.} \partial\left[\sum_{i=1}^{k} \mu_{i} \Phi_{i}\right](x) \\
& =N_{S}(\bar{x}) \quad \text { in view of Theorem } 5.1
\end{aligned}
$$

The proof is complete.

We now recover as a corollary the classical formula for the normal cone $N_{S}(\bar{x})$ by assuming the continuity of the functions $\Phi_{i}, i=1, \ldots, k$, along with the Slater condition (5.9).

Corollary 5.5. Let $X$ be a reflexive Banach space and let $\Phi_{1}, \ldots, \Phi_{k}: X \rightarrow \mathbb{R}$ be continuous convex functions. Given $\lambda_{1}, \ldots, \lambda_{k} \in \mathbb{R}$, let us define the set $S$ by $S=\bigcap_{i=1}^{k}\left[\Phi_{i} \leq \lambda_{i}\right]$. Assume that the Slater condition (5.9) holds true. Then, for $\bar{x} \in S$, we have

$$
N_{S}(\bar{x})=\sum_{i \in I(\bar{x})} \mathbb{R}_{+} \partial \Phi_{i}(\bar{x}),
$$

with $I(\bar{x})=\left\{i \in\{1, \ldots, k\}, \Phi_{i}(\bar{x})=\lambda_{i}\right\}$ and the convention that the sum over an empty set of indices equals zero.

Proof. In view of Theorem [5.3, the following equality holds:

$$
N_{S}(\bar{x})=\limsup _{\left\{\begin{array}{l}
x \rightarrow \bar{x}, \mu_{i} \geq 0 \\
\mu_{i} \rightarrow 0 \text { if } i \notin I(\bar{x})
\end{array}\right.} \partial\left[\sum_{i=1}^{k} \mu_{i} \Phi_{i}\right](x) .
$$

On the other hand, since each function $\Phi_{i}$ is continuous, we have for every $x \in X$,

$$
\partial\left[\sum_{i=1}^{k} \mu_{i} \Phi_{i}\right](x)=\sum_{i=1}^{k} \mu_{i} \partial \Phi_{i}(x)
$$

Hence, we must prove that

$$
\limsup _{\left\{\begin{array}{l}
x \rightarrow \bar{x}, \mu_{i} \geq 0 \\
\mu_{i} \rightarrow 0 \text { if } i \notin I(\bar{x})
\end{array}\right.} \sum_{i=1}^{k} \mu_{i} \partial \Phi_{i}(x)=\sum_{i \in I(\bar{x})} \mathbb{R}_{+} \partial \Phi_{i}(\bar{x}) .
$$

Let $x^{*} \in \limsup _{\left\{\begin{array}{l}x \rightarrow \bar{x}, \mu_{i} \geq 0 \\ \mu_{i} \rightarrow 0 \text { if } i \notin I(\bar{x})\end{array}\right.} \sum_{i=1}^{k} \mu_{i} \partial \Phi_{i}(x)$. By definition, there exist sequences $\left(x_{n}\right) \subset X,\left(x_{i, n}^{*}\right) \subset X^{*},\left(\mu_{i, n}\right) \subset \mathbb{R}_{+}$such that $x_{i, n}^{*} \in \partial \Phi_{i}\left(x_{n}\right), x_{n} \underset{n \rightarrow+\infty}{\longrightarrow} \bar{x}$, $\sum_{i=1}^{k} \mu_{i, n} x_{i, n}^{*} \underset{n \rightarrow+\infty}{\longrightarrow} x^{*}$ and $\mu_{i, n} \underset{n \rightarrow+\infty}{\longrightarrow} 0$ if $i \notin I(\bar{x})$. Since each function $\Phi_{i}$ is continuous, the sequence $\left(x_{i, n}^{*}\right)$ is bounded and hence has a convergent subsequence with respect to the weak-star topology of $X^{*}$. On the other hand, Lemma 5.4 
shows that each sequence $\left(\mu_{i, n}\right)$ is bounded. Therefore, by iteratively extracting subsequences, we can build an increasing map $\varphi: \mathbb{N} \rightarrow \mathbb{N}$ such that

$$
\forall i \in\{1, \ldots, k\}, \quad x_{i, \varphi(n)}^{*} \underset{n \rightarrow+\infty}{\stackrel{w^{*}}{\rightarrow}} x_{i}^{*} \quad \text { and } \quad \mu_{i, \varphi(n)} \underset{n \rightarrow+\infty}{\longrightarrow} \bar{\mu}_{i},
$$

for some $x_{1}^{*}, \ldots, x_{k}^{*} \in X^{*}$ and $\bar{\mu}_{1}, \ldots, \bar{\mu}_{k} \in \mathbb{R}_{+}$. From this and the sequential \|\|$\times w^{*}$ graph-closedness property of each operator $\partial \Phi_{i}$, we immediately obtain that $x_{i}^{*} \in \partial \Phi_{i}(\bar{x})$. Since $\sum_{i=1}^{k} \mu_{i, n} x_{i, n}^{*} \underset{n \rightarrow+\infty}{\longrightarrow} x^{*}$, we deduce from (5.17) that $\sum_{i=1}^{k} \bar{\mu}_{i} x_{i}^{*}=x^{*}$. Recalling that $\bar{\mu}_{i}=0$ for $i \notin I(\bar{x})$, this implies that $x^{*}=$ $\sum_{i \in I(\bar{x})} \bar{\mu}_{i} x_{i}^{*} \in \sum_{i \in I(\bar{x})} \mathbb{R}_{+} \partial \Phi_{i}(\bar{x})$. Therefore the inclusion

$$
\limsup _{\left\{\begin{array}{l}
x \rightarrow \bar{x}, \mu_{i} \geq 0 \\
\mu_{i} \rightarrow 0 \text { if } i \notin I(\bar{x})
\end{array}\right.} \sum_{i=1}^{k} \mu_{i} \partial \Phi_{i}(x) \subset \sum_{i \in I(\bar{x})} \mathbb{R}_{+} \partial \Phi_{i}(\bar{x})
$$

is proved, and since the reverse inclusion is immediate, the proof is complete.

In Theorems 5.1 and 5.3 the normal cone $N_{S}(\bar{x})$ is described through the subdifferential of positively linear combinations of the functions $\Phi_{i}, i=1, \ldots, k$. The next theorem provides an additional description via the separate subdifferentials of the functions $\Phi_{i}$. For such a description we use the set

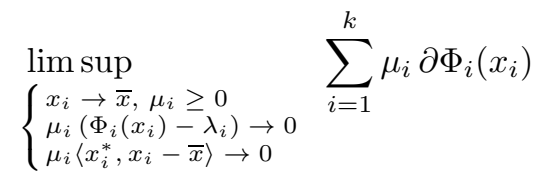

defined as the set of limits $\lim _{n \rightarrow+\infty} \sum_{i=1}^{k} \mu_{i, n} x_{i, n}^{*}$ such that $\mu_{i, n} \geq 0, x_{i, n} \underset{n \rightarrow+\infty}{\longrightarrow} \bar{x}$, $x_{i, n}^{*} \in \partial \Phi_{i}\left(x_{i, n}\right), \mu_{i, n}\left(\Phi_{i}\left(x_{i, n}\right)-\lambda_{i}\right) \underset{n \rightarrow+\infty}{\longrightarrow} 0$ and $\mu_{i, n}\left\langle x_{i, n}^{*}, x_{i, n}-\bar{x}\right\rangle \underset{n \rightarrow+\infty}{\longrightarrow} 0$ for $i=1, \ldots, k$. In the same vein, we denote by

$$
\limsup _{\left\{\begin{array}{l}
x_{i} \rightarrow \bar{x}, \mu_{i} \geq 0 \\
\mu_{i}\left(\Phi_{i}\left(x_{i}\right)-\lambda_{i}-\left\langle x_{i}^{*}, x_{i}-\bar{x}\right\rangle\right) \rightarrow 0
\end{array}\right.} \sum_{i=1}^{k} \mu_{i} \partial \Phi_{i}\left(x_{i}\right)
$$

and respectively

$$
\limsup _{\left\{\begin{array}{l}
x_{i} \rightarrow \bar{x}, \mu_{i} \geq 0 \\
\sum_{i=1}^{k} \mu_{i}\left(\Phi_{i}\left(x_{i}\right)-\lambda_{i}-\left\langle x_{i}^{*}, x_{i}-\bar{x}\right\rangle\right) \rightarrow 0
\end{array}\right.} \sum_{i=1}^{k} \mu_{i} \partial \Phi_{i}\left(x_{i}\right)
$$

the sets, where the last conditions of the above definition are replaced respectively by

$$
\mu_{i, n}\left(\Phi_{i}\left(x_{i, n}\right)-\lambda_{i}-\left\langle x_{i, n}^{*}, x_{i, n}-\bar{x}\right\rangle\right) \underset{n \rightarrow+\infty}{\longrightarrow} 0 \text { for } i=1, \ldots, k
$$

and

$$
\sum_{i=1}^{k} \mu_{i, n}\left(\Phi_{i}\left(x_{i, n}\right)-\lambda_{i}-\left\langle x_{i, n}^{*}, x_{i, n}-\bar{x}\right\rangle\right) \underset{n \rightarrow+\infty}{\longrightarrow} 0 .
$$

By combining Theorems 2.1 and 3.1, we show in the next theorem that the normal cone $N_{S}(\bar{x})$ to the set $S=\bigcap_{i=1}^{k}\left[\Phi_{i} \leq \lambda_{i}\right]$ coincides with the above upper limits. 
Theorem 5.6. Let $X$ be a reflexive Banach space and let $\Phi_{1}, \ldots, \Phi_{k}: X \rightarrow \mathbb{R} \cup$ $\{+\infty\}$ be lower semicontinuous convex functions. Given $\lambda_{1}, \ldots, \lambda_{k} \in \mathbb{R}$, let us define the set $S$ by $S=\bigcap_{i=1}^{k}\left[\Phi_{i} \leq \lambda_{i}\right]$. For $\bar{x} \in S$, we have

$$
\begin{aligned}
& N_{S}(\bar{x})=\lim _{\left\{\begin{array}{l}
x_{i} \rightarrow \bar{x}, \mu_{i} \geq 0 \\
\mu_{i}\left(\Phi_{i}\left(x_{i}\right)-\lambda_{i}\right) \rightarrow 0 \\
\mu_{i}\left\langle x_{i}^{*}, x_{i}-\bar{x}\right\rangle \rightarrow 0
\end{array}\right.} \sum_{i=1}^{k} \mu_{i} \partial \Phi_{i}\left(x_{i}\right) \\
& =\limsup _{\left\{\begin{array}{l}
x_{i} \rightarrow \bar{x}, \mu_{i} \geq 0 \\
\mu_{i}\left(\Phi_{i}\left(x_{i}\right)-\lambda_{i}-\left\langle x_{i}^{*}, x_{i}-\bar{x}\right\rangle\right) \rightarrow 0
\end{array}\right.} \sum_{i=1}^{k} \mu_{i} \partial \Phi_{i}\left(x_{i}\right) \\
& =\limsup _{\left\{\begin{array}{l}
x_{i} \rightarrow \bar{x}, \mu_{i} \geq 0 \\
\sum_{i=1}^{k} \mu_{i}\left(\Phi_{i}\left(x_{i}\right)-\lambda_{i}-\left\langle x_{i}^{*}, x_{i}-\bar{x}\right\rangle\right) \rightarrow 0
\end{array}\right.} \sum_{i=1}^{k} \mu_{i} \partial \Phi_{i}\left(x_{i}\right) .
\end{aligned}
$$

Proof. Let $x^{*} \in N_{S}(\bar{x})$. First observe that $\delta_{S}=\sum_{i=1}^{k} \delta_{\left[\Phi_{i} \leq \lambda_{i}\right]}$, hence

$$
N_{S}(\bar{x})=\partial \delta_{S}(\bar{x})=\partial\left(\sum_{i=1}^{k} \delta_{\left[\Phi_{i} \leq \lambda_{i}\right]}\right)(\bar{x}) .
$$

From Theorem 2.1] there exist sequences $\left(u_{i, n}\right)_{n} \subset X,\left(u_{i, n}^{*}\right)_{n} \subset X^{*}, i \in\{1, \ldots, k\}$, such that $u_{i, n}^{*} \in N_{\left[\Phi_{i} \leq \lambda_{i}\right]}\left(u_{i, n}\right)$ and

$$
u_{i, n} \underset{n \rightarrow+\infty}{\longrightarrow} \bar{x}, \quad \sum_{i=1}^{k} u_{i, n}^{*} \underset{n \rightarrow+\infty}{\longrightarrow} x^{*} \quad \text { and } \quad\left\langle u_{i, n}^{*}, u_{i, n}-\bar{x}\right\rangle \underset{n \rightarrow+\infty}{\longrightarrow} 0 .
$$

In view of Theorem 3.1, we have

$$
N_{\left[\Phi_{i} \leq \lambda_{i}\right]}\left(u_{i, n}\right)=\limsup _{\left\{\begin{array}{l}
x \rightarrow u_{i, n}, \nu \geq 0 \\
\nu\left(\Phi_{i}(x)-\lambda_{i}\right) \rightarrow 0
\end{array}\right.} \nu \partial \Phi_{i}(x) .
$$

Hence there exist sequences $\left(\nu_{i, n, m}\right)_{m} \subset \mathbb{R},\left(v_{i, n, m}\right)_{m} \subset X$ and $\left(v_{i, n, m}^{*}\right)_{m} \subset X^{*}$ such that

$$
\nu_{i, n, m} \geq 0, \quad v_{i, n, m}^{*} \in \partial \Phi_{i}\left(v_{i, n, m}\right)
$$

$$
v_{i, n, m} \underset{m \rightarrow+\infty}{\longrightarrow} u_{i, n}, \quad \nu_{i, n, m} v_{i, n, m}^{*} \underset{m \rightarrow+\infty}{\longrightarrow} u_{i, n}^{*}, \quad \nu_{i, n, m}\left(\Phi_{i}\left(v_{i, n, m}\right)-\lambda_{i}\right) \underset{m \rightarrow+\infty}{\longrightarrow} 0 .
$$

From (5.20), there exists an increasing function $\varphi: \mathbb{N} \rightarrow \mathbb{N}$ such that for every $i \in\{1, \ldots, k\}$ and every $n \in \mathbb{N}$,

$$
\left\|v_{i, n, \varphi(n)}-u_{i, n}\right\| \leq \frac{1}{n}, \quad\left|\left\langle u_{i, n}^{*}, v_{i, n, \varphi(n)}-u_{i, n}\right\rangle\right| \leq \frac{1}{n},
$$

$$
\left\|\nu_{i, n, \varphi(n)} v_{i, n, \varphi(n)}^{*}-u_{i, n}^{*}\right\| \leq \frac{1}{n} \quad \text { and } \quad\left|\nu_{i, n, \varphi(n)}\left(\Phi_{i}\left(v_{i, n, \varphi(n)}\right)-\lambda_{i}\right)\right| \leq \frac{1}{n}
$$


Let us then set $\mu_{i, n}=\nu_{i, n, \varphi(n)}, x_{i, n}=v_{i, n, \varphi(n)}$ and $x_{i, n}^{*}=v_{i, n, \varphi(n)}^{*}$. In view of (5.19), we have $\mu_{i, n} \geq 0$ and $x_{i, n}^{*} \in \partial \Phi_{i}\left(x_{i, n}\right)$. By using (5.18), (5.21) and (5.22), we find

$$
\begin{aligned}
& \left\|x_{i, n}-\bar{x}\right\| \leq\left\|x_{i, n}-u_{i, n}\right\|+\left\|u_{i, n}-\bar{x}\right\| \\
& \leq \frac{1}{n}+\left\|u_{i, n}-\bar{x}\right\| \underset{n \rightarrow+\infty}{\longrightarrow} 0 \text {, } \\
& \left\|\sum_{i=1}^{k} \mu_{i, n} x_{i, n}^{*}-x^{*}\right\| \leq\left\|\sum_{i=1}^{k} \mu_{i, n} x_{i, n}^{*}-\sum_{i=1}^{k} u_{i, n}^{*}\right\|+\left\|\sum_{i=1}^{k} u_{i, n}^{*}-x^{*}\right\| \\
& \leq \sum_{i=1}^{k}\left\|\mu_{i, n} x_{i, n}^{*}-u_{i, n}^{*}\right\|+\left\|\sum_{i=1}^{k} u_{i, n}^{*}-x^{*}\right\| \\
& \leq \frac{k}{n}+\left\|\sum_{i=1}^{k} u_{i, n}^{*}-x^{*}\right\| \underset{n \rightarrow+\infty}{\longrightarrow} 0, \\
& \left|\left\langle\mu_{i, n} x_{i, n}^{*}, x_{i, n}-\bar{x}\right\rangle\right| \leq\left|\left\langle\mu_{i, n} x_{i, n}^{*}-u_{i, n}^{*}, x_{i, n}-u_{i, n}\right\rangle\right| \\
& +\left|\left\langle\mu_{i, n} x_{i, n}^{*}-u_{i, n}^{*}, u_{i, n}-\bar{x}\right\rangle\right| \\
& +\left|\left\langle u_{i, n}^{*}, x_{i, n}-u_{i, n}\right\rangle\right|+\left|\left\langle u_{i, n}^{*}, u_{i, n}-\bar{x}\right\rangle\right| \\
& \leq\left\|\mu_{i, n} x_{i, n}^{*}-u_{i, n}^{*}\right\|\left\|x_{i, n}-u_{i, n}\right\| \\
& +\left\|\mu_{i, n} x_{i, n}^{*}-u_{i, n}^{*}\right\|\left\|u_{i, n}-\bar{x}\right\| \\
& +\left|\left\langle u_{i, n}^{*}, x_{i, n}-u_{i, n}\right\rangle\right|+\left|\left\langle u_{i, n}^{*}, u_{i, n}-\bar{x}\right\rangle\right| \\
& \leq \frac{1}{n^{2}}+\frac{1}{n}\left\|u_{i, n}-\bar{x}\right\|+\frac{1}{n}+\left|\left\langle u_{i, n}^{*}, u_{i, n}-\bar{x}\right\rangle\right| \underset{n \rightarrow+\infty}{\longrightarrow} 0,
\end{aligned}
$$

and

$$
\left|\mu_{i, n}\left(\Phi_{i}\left(x_{i, n}\right)-\lambda_{i}\right)\right| \leq \frac{1}{n}
$$

Finally, we have built sequences $\left(x_{i, n}\right)_{n} \subset X,\left(x_{i, n}^{*}\right)_{n} \subset X^{*},\left(\mu_{i, n}\right)_{n} \subset \mathbb{R}_{+}$, $i \in\{1, \ldots, k\}$, such that $x_{i, n}^{*} \in \partial \Phi_{i}\left(x_{i, n}\right)$ with $x_{i, n} \underset{n \rightarrow+\infty}{\longrightarrow} \bar{x}, \sum_{i=1}^{k} \mu_{i, n} x_{i, n}^{*} \underset{n \rightarrow+\infty}{\longrightarrow} x^{*}$, $\mu_{i, n}\left\langle x_{i, n}^{*}, x_{i, n}-\bar{x}\right\rangle \underset{n \rightarrow+\infty}{\longrightarrow} 0$ and $\mu_{i, n}\left(\Phi_{i}\left(x_{i, n}\right)-\lambda_{i}\right) \underset{n \rightarrow+\infty}{\longrightarrow} 0$. Hence we obtain that $x^{*} \in \limsup _{\left\{\begin{array}{l}x_{i} \rightarrow \bar{x}, \mu_{i} \geq 0 \\ \mu_{i}\left(\Phi_{i}\left(x_{i}\right)-\lambda_{i}\right) \rightarrow 0 \\ \mu_{i}\left\langle x_{i}^{*}, x_{i}-\bar{x}\right\rangle \rightarrow 0\end{array}\right.} \sum_{i=1}^{k} \mu_{i} \partial \Phi_{i}\left(x_{i}\right)$, which proves the inclusion

$$
N_{S}(\bar{x}) \subset \lim _{\left\{\begin{array}{l}
x_{i} \rightarrow \bar{x}, \mu_{i} \geq 0 \\
\mu_{i}\left(\Phi_{i}\left(x_{i}\right)-\lambda_{i}\right) \rightarrow 0 \\
\mu_{i}\left\langle x_{i}^{*}, x_{i}-\bar{x}\right\rangle \rightarrow 0
\end{array}\right.} \sum_{i=1}^{k} \mu_{i} \partial \Phi_{i}\left(x_{i}\right)
$$


The inclusions

$$
\begin{aligned}
& \limsup _{\substack{x_{i} \rightarrow \bar{x}, \mu_{i} \geq 0 \\
\mu_{i}\left(\Phi_{i}\left(x_{i}\right)-\lambda_{i}\right) \rightarrow 0 \\
\mu_{i}\left\langle x_{i}^{*}, x_{i}-\bar{x}\right\rangle \rightarrow 0}} \sum_{i=1}^{k} \mu_{i} \partial \Phi_{i}\left(x_{i}\right) \\
& \subset \limsup _{\left\{\begin{array}{l}
x_{i} \rightarrow \bar{x}, \mu_{i} \geq 0 \\
\mu_{i}\left(\Phi_{i}\left(x_{i}\right)-\lambda_{i}-\left\langle x_{i}^{*}, x_{i}-\bar{x}\right\rangle\right) \rightarrow 0
\end{array}\right.} \sum_{i=1}^{k} \mu_{i} \partial \Phi_{i}\left(x_{i}\right) \\
& \subset \limsup _{\left\{\begin{array}{l}
x_{i} \rightarrow \bar{x}, \mu_{i} \geq 0 \\
\sum_{i=1}^{k} \mu_{i}\left(\Phi_{i}\left(x_{i}\right)-\lambda_{i}-\left\langle x_{i}^{*}, x_{i}-\bar{x}\right\rangle\right) \rightarrow 0
\end{array}\right.} \sum_{i=1}^{k} \mu_{i} \partial \Phi_{i}\left(x_{i}\right)
\end{aligned}
$$

are obvious. It remains to prove that

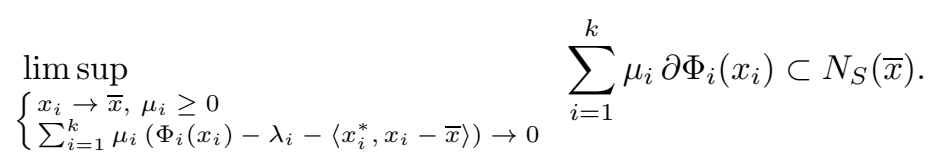

For that purpose, let us assume that $x^{*} \in X^{*}$ is such that $\sum_{i=1}^{k} \mu_{i, n} x_{i, n}^{*} \underset{n \rightarrow+\infty}{\longrightarrow} x^{*}$ with $\mu_{i, n} \geq 0, x_{i, n}^{*} \in \partial \Phi_{i}\left(x_{i, n}\right), \quad x_{i, n} \underset{n \rightarrow+\infty}{\longrightarrow} \bar{x}$ and

$$
\sum_{i=1}^{k} \mu_{i, n}\left(\Phi_{i}\left(x_{i, n}\right)-\lambda_{i}-\left\langle x_{i, n}^{*}, x_{i, n}-\bar{x}\right\rangle\right) \underset{n \rightarrow+\infty}{\longrightarrow} 0 .
$$

Let us fix $x \in S$. Since $x_{i, n}^{*} \in \partial \Phi_{i}\left(x_{i, n}\right)$ and since $\Phi_{i}$ is convex, we have

$$
\begin{aligned}
\left\langle x_{i, n}^{*}, x-x_{i, n}\right\rangle & \leq \Phi_{i}(x)-\Phi_{i}\left(x_{i, n}\right) \\
& \leq \lambda_{i}-\Phi_{i}\left(x_{i, n}\right) \quad \text { because } x \in S .
\end{aligned}
$$

This can be rewritten as

$$
\left\langle x_{i, n}^{*}, x-\bar{x}\right\rangle \leq \lambda_{i}-\Phi_{i}\left(x_{i, n}\right)+\left\langle x_{i, n}^{*}, x_{i, n}-\bar{x}\right\rangle .
$$

After multiplication by $\mu_{i, n}$ and summation from $i=1$ to $k$, we find

$$
\left\langle\sum_{i=1}^{k} \mu_{i, n} x_{i, n}^{*}, x-\bar{x}\right\rangle \leq \sum_{i=1}^{k} \mu_{i, n}\left(\lambda_{i}-\Phi_{i}\left(x_{i, n}\right)+\left\langle x_{i, n}^{*}, x_{i, n}-\bar{x}\right\rangle\right) .
$$

Recalling formula (5.23) and the fact that $\sum_{i=1}^{k} \mu_{i, n} x_{i, n}^{*} \underset{n \rightarrow+\infty}{\longrightarrow} x^{*}$, we obtain at the limit as $n \rightarrow+\infty$ that $\left\langle x^{*}, x-\bar{x}\right\rangle \leq 0$. Since this is true for every $x \in S$, we conclude that $x^{*} \in N_{S}(\bar{x})$. The proof is complete.

\section{EXTENSION TO THE NONCONVEX FRAMEWORK}

In this section, we assume that $X=\mathbb{R}^{N}$ and that the function $\Phi: \mathbb{R}^{N} \rightarrow \mathbb{R}$ is locally Lipschitz continuous. Our purpose is to find a suitable extension of the normal cone formula (3.3) in the nonconvex framework. It is worthwhile noticing at first that equality (3.3) may no longer be true for nonconvex functions. Take for example the function $\Phi: \mathbb{R} \rightarrow \mathbb{R}$ defined by $\Phi(x)=-x^{2}$ and let $\bar{x}=0$. We have 
$S=[\Phi \leq 0]=\mathbb{R}$ and $N_{S}(\bar{x})=N_{\mathbb{R}}(0)=\{0\}$, while

$$
\limsup _{x \rightarrow \bar{x}} \mathbb{R}_{+} \partial \Phi(x)=\limsup _{x \rightarrow 0} \mathbb{R}_{+} \Phi^{\prime}(x)=\mathbb{R},
$$

because $\Phi^{\prime}(x)<0$ (resp. $>0$ ) for $x>0$ (resp. $x<0$ ).

Consider a function $\Phi: \mathbb{R}^{N} \rightarrow \mathbb{R} \cup\{+\infty\}$ and a point $\bar{x}$ with $\Phi(\bar{x})$ finite. We recall that, for a vector $x^{*} \in \mathbb{R}^{N}$, one says that

(i) $x^{*}$ is a Fréchet subgradien 5 of $\Phi$ at $\bar{x}$, written $x^{*} \in \partial_{\mathcal{F}} \Phi(\bar{x})$ if

$$
\liminf _{x \rightarrow \bar{x}, x \neq \bar{x}} \frac{\Phi(x)-\Phi(\bar{x})-\left\langle x^{*}, x-\bar{x}\right\rangle}{\|x-\bar{x}\|} \geq 0 .
$$

(ii) $x^{*}$ is a Mordukhovich limiting subgradient of $\Phi$ at $\bar{x}$, written $x^{*} \in \partial_{L} \Phi(\bar{x})$ if there are sequences $x_{n} \rightarrow_{\Phi} \bar{x}$ and $x_{n}^{*} \in \partial_{\mathcal{F}} \Phi\left(x_{n}\right)$ with $x_{n}^{*} \rightarrow x^{*}$.

Let $C \subset \mathbb{R}^{N}$ and $\bar{x} \in C$. The Fréchet (resp. limiting) normal cone of $C$ at $\bar{x}$ denoted by $N^{\mathcal{F}}(C ; \bar{x})$ (resp. $N^{L}(C ; \bar{x})$ ) is defined by $N^{\mathcal{F}}(C ; \bar{x})=\partial_{\mathcal{F}} \delta_{C}(\bar{x})$ (resp. $\left.N^{L}(C ; \bar{x})=\partial_{L} \delta_{C}(\bar{x})\right)$. The set $C$ is said to be normally regular at $\bar{x}$ if it is locally closed at $\bar{x}$ and $N^{\mathcal{F}}(C ; \bar{x})=N^{L}(C ; \bar{x})$. In finite dimensions, the normal regularity of a set coincides with the Clarke regularity, requiring the equality between the Clarke tangent cone and the Bouligand tangent cone (see 32 ). The concepts are different in any infinite dimensional space (see [12,28]). A function $\Phi: \mathbb{R}^{N} \rightarrow \mathbb{R} \cup\{+\infty\}$ is called subdifferentially subregular at $\bar{x} \in \operatorname{dom} \Phi$ if epi $\Phi$ is normally regular at $(\bar{x}, \Phi(\bar{x}))$ as a subset of $\mathbb{R}^{N} \times \mathbb{R}$. When the function $\Phi$ is locally Lipschitz continuous, the latter condition amounts to the equality $\partial_{L} \Phi(\bar{x})=\partial_{\mathcal{F}} \Phi(\bar{x})$. For standard references on nonsmooth analysis, see for example [9, 11, 20, 21, 28, 32 .

A powerful sum rule (see [28, 32]) is available for the Fréchet subgradients: If $\Phi_{1}, \Phi_{2}: \mathbb{R}^{N} \rightarrow \mathbb{R} \cup\{+\infty\}$ are lower semicontinuous and finite at $\bar{x}$ and if $x^{*} \in$ $\partial_{\mathcal{F}}\left(\Phi_{1}+\Phi_{2}\right)(\bar{x})$, then for every real $\varepsilon>0$ there exist $x_{i} \in \mathbb{R}^{N}$ with $\left\|x_{i}-\bar{x}\right\| \leq \varepsilon$ and $\left|\Phi_{i}\left(x_{i}\right)-\Phi(\bar{x})\right| \leq \varepsilon, i=1,2$, such that

$$
x^{*} \in \partial_{\mathcal{F}} \Phi_{1}\left(x_{1}\right)+\partial_{\mathcal{F}} \Phi_{2}\left(x_{2}\right)+\varepsilon \mathbb{B},
$$

where $\mathbb{B}$ denotes the closed unit ball of $\mathbb{R}^{N}$. From the latter fuzzy sum rule, we deduce a chain rule for the composition of a locally Lipschitz mapping $F$ with a lower semicontinuous function $f$. The next theorem is strongly related 6 to 29 , Theorem 4.10], and it could be derived as a corollary of this result. For the sake of completeness we provide a self-contained proof.

Theorem 6.1. Let $F$ be a locally Lipschitz mapping from $\mathbb{R}^{N}$ into $\mathbb{R}^{m}$ and $f$ : $\mathbb{R}^{m} \rightarrow \mathbb{R} \cup\{+\infty\}$ be a lower semicontinuous function which is finite at $F(\bar{x})$, where $\bar{x} \in \mathbb{R}^{N}$. Then for any $x^{*} \in \partial_{L}(f \circ F)(\bar{x})$, there exist sequences $\left(x_{n}\right) \subset \mathbb{R}^{N}$, $\left(y_{n}\right) \subset \mathbb{R}^{m},\left(x_{n}^{*}\right) \subset \mathbb{R}^{N}$ and $\left(y_{n}^{*}\right) \subset \mathbb{R}^{m}$ such that $x_{n} \rightarrow \bar{x}, y_{n} \rightarrow F(\bar{x}), f\left(y_{n}\right) \rightarrow$ $f(F(\bar{x})), x_{n}^{*} \rightarrow x^{*}$ and

$$
y_{n}^{*} \in \partial_{\mathcal{F}} f\left(y_{n}\right) \quad \text { and } \quad x_{n}^{*} \in \partial_{\mathcal{F}}\left(y_{n}^{*} \circ F\right)\left(x_{n}\right) .
$$

Proof. Consider any $x^{*} \in \partial_{L}(f \circ F)(\bar{x})$ and take an open neighborhood $V_{0}$ of $\bar{x}$ over which $F$ is Lipschitz with $\gamma \geq 0$ as a Lipschitz constant. Fix any real $\varepsilon>0$. Taking, if necessary, another positive real less than $\varepsilon$, we may suppose that $B(\bar{x}, 4 \varepsilon) \subset V_{0}$, where $B(\bar{x}, 4 \varepsilon)$ denotes the open ball centered at $\bar{x}$ with radius $4 \varepsilon$. There exist $z \in \mathbb{R}^{N}$ with $\|z-\bar{x}\| \leq \varepsilon$ and $|f \circ F(z)-f \circ F(\bar{x})| \leq \varepsilon$, and $z^{*} \in \partial_{\mathcal{F}}(f \circ F)(z)$

\footnotetext{
${ }^{5}$ It is also called a lower or regular subgradient in 28 .32].

${ }^{6}$ We thank the referee who indicated to us the reference [29].
} 
with $\left\|z^{*}-x^{*}\right\| \leq \varepsilon$. Put $f_{1}(x, y):=f(y)$ and $f_{2}(x, y):=\delta_{\operatorname{gph} F}(x, y)$ for all $(x, y) \in \mathbb{R}^{N} \times \mathbb{R}^{m}$. It is not difficult to see that $\left(z^{*}, 0\right) \in \partial_{\mathcal{F}}\left(f_{1}+f_{2}\right)(z, F(z))$. Both functions $f_{1}$ and $f_{2}$ are lower semicontinuous. The inclusion (6.1) above combined with the equality $\partial_{\mathcal{F}} f_{1}(x, y)=\{0\} \times \partial_{\mathcal{F}} f(y)$ says that there exist

$$
\begin{aligned}
& (x, y) \in B((z, F(z)), \varepsilon) \text { with }|f(y)-f(F(z))| \leq \varepsilon, \\
& \left(z^{\prime}, F\left(z^{\prime}\right)\right) \in B((z, F(z)), \varepsilon),
\end{aligned}
$$

and

$$
\left(0, y^{*}\right) \in \partial_{\mathcal{F}} f_{1}(x, y), \quad\left(u^{*},-v^{*}\right) \in N^{\mathcal{F}}\left(\operatorname{gph} F ;\left(z^{\prime}, F\left(z^{\prime}\right)\right)\right)
$$

such that

$$
\left(z^{*}, 0\right) \in\left(0, y^{*}\right)+\left(u^{*},-v^{*}\right)+\varepsilon \mathbb{B},
$$

hence $z^{*} \in u^{*}+\varepsilon \mathbb{B}$ and $v^{*} \in y^{*}+\varepsilon \mathbb{B}$. Consequently, we have the inclusion $y^{*} \in$ $\partial_{\mathcal{F}} f(y)$ with $\|y-F(\bar{x})\| \leq \varepsilon(1+\gamma)$ and $|f(y)-f(F(\bar{x}))| \leq 2 \varepsilon$, along with the equality $v^{*}=y^{*}+e^{*}$ for some $e^{*} \in \varepsilon \mathbb{B}$. The inclusion $\left(u^{*},-v^{*}\right) \in N^{\mathcal{F}}\left(\operatorname{gph} F,\left(z^{\prime}, F\left(z^{\prime}\right)\right)\right)$ means that, for any real $\eta>0$, there exists some open neighborhood $V \subset V_{0}$ of $z^{\prime}$ such that, for all $x^{\prime} \in V$,

$$
\left\langle u^{*}, x^{\prime}-z^{\prime}\right\rangle-\left\langle v^{*}, F\left(x^{\prime}\right)-F\left(z^{\prime}\right)\right\rangle \leq \eta\left(\left\|x^{\prime}-z^{\prime}\right\|+\left\|F\left(x^{\prime}\right)-F\left(z^{\prime}\right)\right\|\right),
$$

So

$$
\left\langle u^{*}, x^{\prime}-z^{\prime}\right\rangle \leq\left(v^{*} \circ F\right)\left(x^{\prime}\right)-\left(v^{*} \circ F\right)\left(z^{\prime}\right)+\eta(1+\gamma)\left\|x^{\prime}-z^{\prime}\right\| .
$$

This guarantees that $u^{*} \in \partial_{\mathcal{F}}\left(v^{*} \circ F\right)\left(z^{\prime}\right)$ and hence $u^{*} \in \partial_{\mathcal{F}}\left(y^{*} \circ F+e^{*} \circ F\right)\left(z^{\prime}\right)$. Then, the inclusion (6.1) gives $\xi, \zeta \in \mathbb{R}^{N}$ with $\left\|\xi-z^{\prime}\right\| \leq \varepsilon$ and $\left\|\zeta-z^{\prime}\right\| \leq \varepsilon$ such that

$$
u^{*} \in \partial_{\mathcal{F}}\left(y^{*} \circ F\right)(\xi)+\partial_{\mathcal{F}}\left(e^{*} \circ F\right)(\zeta)+\varepsilon \mathbb{B} .
$$

The inequality $\left\|\xi-z^{\prime}\right\| \leq \varepsilon$ combined with the inequalities $\left\|z^{\prime}-z\right\| \leq \varepsilon$ and $\|z-\bar{x}\| \leq \varepsilon$ entails that $\|\xi-\bar{x}\| \leq 3 \varepsilon$, and in the same way $\|\zeta-\bar{x}\| \leq 3 \varepsilon$. Observing that the function $e^{*} \circ F$ is Lipschitz continuous on $B(\bar{x}, 4 \varepsilon)$ with $\varepsilon \gamma$ as a Lipschitz constant, we have $\partial_{\mathcal{F}}\left(e^{*} \circ F\right)(\zeta) \subset \varepsilon \gamma \mathbb{B}$, hence

$$
u^{*} \in \partial_{\mathcal{F}}\left(y^{*} \circ F\right)(\xi)+\varepsilon(1+\gamma) \mathbb{B} .
$$

Therefore there exists $\xi^{*} \in \partial_{\mathcal{F}}\left(y^{*} \circ F\right)(\xi)$ such that $\left\|\xi^{*}-u^{*}\right\| \leq \varepsilon(1+\gamma)$. Since $\left\|u^{*}-z^{*}\right\| \leq \varepsilon$ and $\left\|z^{*}-x^{*}\right\| \leq \varepsilon$, we deduce $\left\|\xi^{*}-x^{*}\right\| \leq \varepsilon(3+\gamma)$. Finally, we have proved that for every $\varepsilon>0$, there exist $\xi \in \mathbb{R}^{N}$ with $\|\xi-\bar{x}\| \leq 3 \varepsilon$, $y \in \mathbb{R}^{m}$ with $\|y-F(\bar{x})\| \leq \varepsilon(1+\gamma)$ and $|f(y)-f(F(\bar{x}))| \leq 2 \varepsilon, \quad y^{*} \in \partial_{\mathcal{F}} f(y)$ and $\xi^{*} \in \partial_{\mathcal{F}}\left(y^{*} \circ F\right)(\xi)$ such that $\left\|\xi^{*}-x^{*}\right\| \leq \varepsilon(3+\gamma)$. By taking $\varepsilon=1 / n$, we easily build the sequences satisfying the requirements of the statement.

We can now establish our first nonconvex normal cone formula for the sublevel set.

Theorem 6.2. Let $\Phi: \mathbb{R}^{N} \rightarrow \mathbb{R}$ be a locally Lipschitz continuous function and let $\bar{x} \in \mathbb{R}^{N}$ and $S=[\Phi \leq \Phi(\bar{x})]$. Then one has

$$
N^{L}(S ; \bar{x}) \subset \limsup _{x \rightarrow \bar{x}} \mathbb{R}_{+} \partial_{\mathcal{F}} \Phi(x) .
$$


Proof. As in the proof of Theorem 3.1, put $\lambda:=\Phi(\bar{x})$ and define the locally Lipschitz continuous function $F: \mathbb{R}^{N} \rightarrow \mathbb{R}$ by $F(x)=\Phi(x)-\lambda$. The equality $S=\left\{x \in \mathbb{R}^{N}, F(x) \in \mathbb{R}_{-}\right\}$tells us that $\delta_{S}=\delta_{\mathbb{R}_{-}} \circ F$, so

$$
N^{L}(S ; \bar{x})=\partial_{L} \delta_{S}(\bar{x})=\partial_{L}\left(\delta_{\mathbb{R}_{-}} \circ F\right)(\bar{x}) .
$$

Further, the function $\delta_{\mathbb{R}_{-}}$is of course lower semicontinuous. Therefore, fixing $x^{*} \in$ $N^{L}(S ; \bar{x})$, we can apply the above chain rule with $f=\delta_{\mathbb{R}_{-}}$and the function $F$ defined above. We obtain the existence of sequences $\left(x_{n}\right) \subset \mathbb{R}^{N},\left(y_{n}\right) \subset \mathbb{R},\left(x_{n}^{*}\right) \subset$ $\mathbb{R}^{N}$, and $\left(y_{n}^{*}\right) \subset \mathbb{R}$ such that $x_{n} \rightarrow \bar{x}, y_{n} \rightarrow \Phi(\bar{x})-\lambda, x_{n}^{*} \rightarrow x^{*}$ and

$$
\begin{aligned}
& y_{n}^{*} \in N^{\mathcal{F}}\left(\mathbb{R}_{-} ; y_{n}\right)=N_{\mathbb{R}_{-}}\left(y_{n}\right), \quad \text { hence } y_{n}^{*} \geq 0, \\
& x_{n}^{*} \in \partial_{\mathcal{F}}\left(y_{n}^{*} \Phi\right)\left(x_{n}\right)=y_{n}^{*} \partial_{\mathcal{F}} \Phi\left(x_{n}\right) .
\end{aligned}
$$

This clearly justifies the desired inclusion.

When the locally Lipschitz continuous function $\Phi$ is subdifferentially subregular, the above result can be strengthened by requiring that the points $x$ converging to $\bar{x}$ stay outside the sublevel set $S$. To achieve that property, let us start with a result that follows immediately from a nonsmooth version of the Sard theorem.

Lemma 6.3. Let $\Phi: \mathbb{R}^{N} \rightarrow \mathbb{R}$ be a locally Lipschitz continuous function. Given any $\bar{\lambda} \in \mathbb{R}$, there exists a decreasing sequence $\left(\lambda_{n}\right) \subset \mathbb{R}$ such that $\lim _{n \rightarrow+\infty} \lambda_{n}=\bar{\lambda}$ and such that for every $x \in \Phi^{-1}\left(\lambda_{n}\right), \Phi$ is differentiable at $x$ with $\nabla \Phi(x) \neq 0$.

Proof. Let $\Omega \subset \Phi\left(\mathbb{R}^{N}\right)$ consist of the values $\lambda \in \mathbb{R}$ such that, for every $x \in \Phi^{-1}(\lambda)$, $\Phi$ is differentiable at $x$ and $\nabla \Phi(x) \neq 0$. Let us also define the set $\widehat{\Omega}$ by

$$
\begin{aligned}
\widehat{\Omega} & =\Omega \cup\left[\mathbb{R} \backslash \Phi\left(\mathbb{R}^{N}\right)\right] \\
& =\left\{\lambda \in \mathbb{R}, \forall x \in \Phi^{-1}(\lambda), \Phi \text { is differentiable at } x \text { and } \nabla \Phi(x) \neq 0\right\} .
\end{aligned}
$$

Notice that the set $\Phi^{-1}(\lambda)$ may be empty in the definition of $\widehat{\Omega}$. From a Lipschitzian version of the Sard theorem, we know that $\Phi\left(\mathbb{R}^{N}\right) \backslash \Omega$ is negligible; see [27] or [32, Theorem 9.65]. It ensues that $\operatorname{int}\left(\Phi\left(\mathbb{R}^{N}\right) \backslash \Omega\right)=\emptyset$, or equivalently $\operatorname{cl}(\widehat{\Omega})=\mathbb{R}$. Given any $\bar{\lambda} \in \mathbb{R}$, it is then easy to build a decreasing sequence $\left(\lambda_{n}\right) \subset \widehat{\Omega}$ such that $\lim _{n \rightarrow+\infty} \lambda_{n}=\bar{\lambda}$.

Under the qualification condition $0 \notin \partial_{L} \Phi(x)$ and the subdifferential subregularity of the locally Lipschitz continuous function $\Phi$, the following was already known; see for example [20, Theorem 2.4.7] or [32, Proposition 10.3].

Lemma 6.4. Let $\Phi: \mathbb{R}^{N} \rightarrow \mathbb{R}$ be a locally Lipschitz continuous function. Given $x \in \mathbb{R}^{N}$, let us consider the level set $S=[\Phi \leq \Phi(x)]$. If $\Phi$ is subdifferentially subregular at $x$ with $0 \notin \partial_{L} \Phi(x)$, then the set $S$ is normally regular at $x$ and $N^{L}(S ; x)=\mathbb{R}_{+} \partial_{L} \Phi(x)$.

Let us finally recall a result of approximation for normals; see [32, Exercise 6.18]. It can be seen as a nonconvex version of $(i i)$ in Theorem 2.4

Lemma 6.5. Let $C$ and $C_{n}, n \in \mathbb{N}$, be nonempty closed sets of $\mathbb{R}^{N}$. Assume that the sequence $\left(C_{n}\right)$ converges toward $C$ in the sense of Painlevé-Kuratowski. Then, one has

$$
\operatorname{gph} N^{L}(C ; \cdot) \subset \liminf _{n \rightarrow+\infty}\left(\operatorname{gph} N^{L}\left(C_{n} ; \cdot\right)\right) .
$$

We can now state the second main result of this section. 
Theorem 6.6. Let $\Phi: \mathbb{R}^{N} \rightarrow \mathbb{R}$ be a locally Lipschitz continuous function, which is subdifferentially subregular near $\bar{x} \in \mathbb{R}^{N}$. Let us consider the set $S=[\Phi \leq \Phi(\bar{x})]$. If $\bar{x} \notin \operatorname{int}(S)$, then we hav 7

$$
N^{L}(S ; \bar{x}) \subset \limsup _{x \rightarrow \bar{x}, x \in D \backslash S} \mathbb{R}_{+} \nabla \Phi(x),
$$

where $D$ denotes the set of $x \in \mathbb{R}^{N}$ where $\Phi$ is differentiable at $x$.

Proof. Since $\Phi$ is locally Lipschitz continuous, Rademacher's theorem asserts that $\Phi$ is differentiable almost everywhere on the open set $\mathbb{R}^{N} \backslash S$. This implies that $D \backslash S$ is dense in $\mathbb{R}^{N} \backslash S$, i.e. $\operatorname{cl}(D \backslash S) \supset \mathbb{R}^{N} \backslash S$, and hence $\operatorname{cl}(D \backslash S) \supset \operatorname{cl}\left(\mathbb{R}^{N} \backslash S\right)$. From the assumption $\bar{x} \notin \operatorname{int}(S)$, we have $\bar{x} \in \operatorname{cl}\left(\mathbb{R}^{N} \backslash S\right)$, and therefore $\bar{x} \in \operatorname{cl}(D \backslash S)$. This tells us that the upper limit arising in (6.3) is well defined.

Let us now prove formula (6.3). Observing that $0 \in \limsup _{x \rightarrow \bar{x}, x \in D \backslash S} \mathbb{R}_{+} \nabla \Phi(x)$, it suffices to show that

$$
N^{L}(S ; \bar{x}) \backslash\{0\} \subset \limsup _{x \rightarrow \bar{x}, x \in D \backslash S} \mathbb{R}_{+} \nabla \Phi(x) .
$$

Let us fix $x^{*} \in N^{L}(S ; \bar{x}) \backslash\{0\}$. Let us set $\bar{\lambda}=\Phi(\bar{x})$ and consider the decreasing sequence $\left(\lambda_{n}\right) \subset \mathbb{R}$ given by Lemma 6.3. Let us define the set $S_{n} \subset \mathbb{R}^{N}$ by $S_{n}=\left[\Phi \leq \lambda_{n}\right]$ for every $n \in \mathbb{N}$. The sequence $\left(S_{n}\right)$ is nonincreasing and the sets $S_{n}$ are closed, hence

$$
\lim _{n \rightarrow+\infty} S_{n}=\bigcap_{n \in \mathbb{N}} \operatorname{cl}\left(S_{n}\right)=\bigcap_{n \in \mathbb{N}} S_{n}=[\Phi \leq \Phi(\bar{x})]=S .
$$

Lemma 6.5 then shows that there exist a sequence $\left(x_{n}\right) \subset \mathbb{R}^{N}$ and a sequence $\left(x_{n}^{*}\right) \subset \mathbb{R}^{N}$ such that $x_{n} \in S_{n}, x_{n}^{*} \in N^{L}\left(S_{n} ; x_{n}\right), x_{n} \rightarrow \bar{x}$ and $x_{n}^{*} \rightarrow x^{*}$ as $n \rightarrow+\infty$. Since $x^{*} \neq 0$, there exists $n_{0} \in \mathbb{N}$ such that $x_{n}^{*} \neq 0$ for every $n \geq n_{0}$. Without loss of generality, we suppose in the sequel that $n \geq n_{0}$. Let us now prove that $\Phi\left(x_{n}\right)=\lambda_{n}$. Let us argue by contradiction and assume that $\Phi\left(x_{n}\right)<\lambda_{n}$. Since $\Phi$ is continuous at $x_{n}$, we deduce that $x_{n} \in \operatorname{int}\left(S_{n}\right)$ and hence $N^{L}\left(S_{n} ; x_{n}\right)=\{0\}$. This implies that $x_{n}^{*}=0$, which gives the contradiction. We have shown that $\Phi\left(x_{n}\right)=\lambda_{n}$, hence in particular $x_{n} \notin S$. From the definition of the sequence $\left(\lambda_{n}\right)$, we have $x_{n} \in D$ and $\nabla \Phi\left(x_{n}\right) \neq 0$; see Lemma 6.3. Since the function $\Phi$ is subdifferentially subregular near $\bar{x}$ by assumption, we infer that, for $n$ large enough, say $n \geq n_{1}$ with $n_{1} \geq n_{0}$,

$$
\partial_{L} \Phi\left(x_{n}\right)=\partial_{\mathcal{F}} \Phi\left(x_{n}\right)=\left\{\nabla \Phi\left(x_{n}\right)\right\}, \text { hence } 0 \notin \partial_{L} \Phi\left(x_{n}\right) .
$$

For each $n \geq n_{1}$, the hypotheses of Lemma 6.4 are satisfied with the point $x=x_{n}$ and the set $S_{n}=\left[\Phi \leq \Phi\left(x_{n}\right)\right]$; thus we obtain that $N^{L}\left(S_{n} ; x_{n}\right)=\mathbb{R}_{+} \nabla \Phi\left(x_{n}\right)$ and hence $x_{n}^{*} \in \mathbb{R}_{+} \nabla \Phi\left(x_{n}\right)$. As a conclusion, we have built a sequence $\left(x_{n}\right)$ such that $x_{n} \in D \backslash S$ for every $n \geq n_{1}$ and $x_{n} \rightarrow \bar{x}$ as $n \rightarrow+\infty$, along with a sequence $\left(x_{n}^{*}\right)$ such that $x_{n}^{*} \in \mathbb{R}_{+} \nabla \Phi\left(x_{n}\right)$ for every $n \geq n_{1}$ and $x_{n}^{*} \rightarrow x^{*}$ as $n \rightarrow+\infty$. This shows that $x^{*} \in \limsup \mathbb{R}_{+} \nabla \Phi(x)$, which ends the proof of the inclusion (6.4).

\footnotetext{
${ }^{7}$ If $\bar{x} \in \operatorname{int}(S)$, we obviously have $N_{S}(\bar{x})=\{0\}$. The assumption $\bar{x} \in \operatorname{int}(S)$ means that $\bar{x}$ is a local maximum for the function $\Phi$.
} 
Remark 6.7. The inclusion (6.3) may be strict if $\Phi$ is not convex. Take for example the function $\Phi: \mathbb{R} \rightarrow \mathbb{R}$ defined by

$$
\Phi(x)= \begin{cases}x^{3}\left(1+\sin ^{2}(1 / x)\right) & \text { if } \quad x>0 \\ 0 & \text { if } \quad x \leq 0\end{cases}
$$

and let $\bar{x}=0$. It is easy to check that the function $\Phi$ is of class $\mathcal{C}^{1}$ on $\mathbb{R}$, hence $\Phi$ is locally Lipschitz continuous and subdifferentially subregular on $\mathbb{R}$. We have $S=\mathbb{R}_{-}$and $N^{L}(S ; \bar{x})=N_{\mathbb{R}_{-}}(0)=\mathbb{R}_{+}$. On the other hand, we find

$$
\limsup _{x \rightarrow \bar{x}, x \in D \backslash S} \mathbb{R}_{+} \nabla \Phi(x)=\limsup _{x \rightarrow 0, x>0} \mathbb{R}_{+} \Phi^{\prime}(x)=\mathbb{R},
$$

since the sign of the derivative $\Phi^{\prime}(x)$ changes infinitely many times as $x \rightarrow 0^{+}$.

\section{Application to The ASYMPtotic STUdy OF SOME GRADIENT-LIKE SYSTEM}

7.1. On a strict obtuseness condition. In this section, we assume that $X=\mathbb{R}^{N}$ and that $S \subset \mathbb{R}^{N}$ is a nonempty closed convex set. Given $\bar{x} \in S$, we focus our attention on the condition

$$
-N_{S}(\bar{x}) \subset \operatorname{int}\left(T_{S}(\bar{x})\right) \cup\{0\}
$$

where $T_{S}(\bar{x})$ is the tangent cone defined by $T_{S}(\bar{x})=\operatorname{cl}\left[\cup_{\lambda>0} \lambda(S-\bar{x})\right]$. The convex cones $N_{S}(\bar{x})$ and $T_{S}(\bar{x})$ are polar to each other, i.e. $N_{S}(\bar{x})=\left[T_{S}(\bar{x})\right]^{*}$ and $T_{S}(\bar{x})=$ $\left[N_{S}(\bar{x})\right]^{*}$. The polar cone $K^{*}$ of a cone $K \subset \mathbb{R}^{N}$ is defined by

$$
K^{*}=\left\{x^{*} \in \mathbb{R}^{N}, \quad \forall x \in K, \quad\left\langle x^{*}, x\right\rangle \leq 0\right\} .
$$

A closed convex cone $K \subset \mathbb{R}^{N}$ is said to be obtuse if $K \supset-K^{*}$. The condition (7.1) amounts to saying that the cone $T_{S}(\bar{x})$ is strictly obtuse, which means that the set $S$ is locally strictly obtuse at $\bar{x}$. Assumption (7.1) entails that $\operatorname{int}(S) \neq \emptyset$. Indeed, if $\operatorname{int}(S)=\emptyset$, then we have $\operatorname{int}\left(T_{S}(\bar{x})\right)=\emptyset$. Condition (7.1) then implies that $N_{S}(\bar{x})=\{0\}$, which implies in our context that $\bar{x} \in \operatorname{int}(S)$, a contradiction. Condition (7.1) is satisfied in particular if $\bar{x}$ is an interior point of $S$ or if $\bar{x}$ is a boundary point of $S$ such that $S$ is smooth 8 at $\bar{x}$. Before stating the main result, let us establish some preliminary lemmas.

Lemma 7.1. Let $S \subset \mathbb{R}^{N}$ be a nonempty closed convex set. Given $\bar{x} \in S$, assume that condition (7.1) is satisfied. Then there exists a convex cone $K \subset \mathbb{R}^{N}$ which is closed and pointed.9 such that

$$
K \subset \operatorname{int}\left(T_{S}(\bar{x})\right) \cup\{0\}
$$

and

$$
-N_{S}(\bar{x}) \subset \operatorname{int}(K) \cup\{0\} .
$$

Proof. If $\bar{x} \in \operatorname{int}(S)$, it suffices to take $K=\{0\}$. Now assume that $\bar{x} \in \operatorname{bd}(S)$. Let us define the set $K$ by

$$
K=\left\{x \in \mathbb{R}^{N}, \quad d\left(x,-N_{S}(\bar{x})\right) \leq d\left(x, \mathbb{R}^{N} \backslash T_{S}(\bar{x})\right)\right\} .
$$

\footnotetext{
${ }^{8} \mathrm{~A}$ convex set $S$ is smooth at $\bar{x} \in \operatorname{bd}(S)$ if there exists $d \neq 0$ such that $N_{S}(\bar{x})=\mathbb{R}_{+} d$.

${ }^{9} \mathrm{~A}$ convex cone $K$ is said to be pointed if $K \cap(-K)=\{0\}$.
} 
It is immediate to check that the set $K$ is a closed cone satisfying (7.2)-(7.3). Since $\bar{x} \in \operatorname{bd}(S)$, there exists $u \in \mathbb{R}^{N} \backslash\{0\}$ such that $\mathbb{R}_{+} u \subset N_{S}(\bar{x})$. By polarity, we have $T_{S}(\bar{x}) \subset\left\{x \in \mathbb{R}^{N},\langle x, u\rangle \leq 0\right\}$, hence

$$
K \subset\left\{x \in \mathbb{R}^{N}, \quad\langle x, u\rangle<0\right\} \cup\{0\} .
$$

It ensues that the cone $K$ is pointed. To prove the convexity of the set $K$, we resort to the following claim.

Claim 7.2. Let $C \subset \mathbb{R}^{N}$ be a nonempty convex set. Then the following hold:

(i) The function $d(\cdot, C)$ is convex on $\mathbb{R}^{N}$.

(ii) If $C \neq \mathbb{R}^{N}$, the function $d\left(\cdot, \mathbb{R}^{N} \backslash C\right)$ is concave on $C$.

The first point is elementary. The second one is given as an exercise by N. Bourbaki [13, Exercise 18, p. 150]; see also [22. We deduce from this claim that the function $\Delta=d\left(.,-N_{S}(\bar{x})\right)-d\left(., \mathbb{R}^{N} \backslash T_{S}(\bar{x})\right)$ is convex on $T_{S}(\bar{x})$. In view of formula (17.2), we have $K \subset T_{S}(\bar{x})$ and we infer that the set $K=\left\{x \in T_{S}(\bar{x}), \Delta(x) \leq 0\right\}$ is convex as a sublevel set of the convex function $\Delta$.

Lemma 7.3. Let $S \subset \mathbb{R}^{N}$ be a nonempty closed convex set and let $\bar{x} \in S$. Let $K \subset \mathbb{R}^{N}$ be a closed cone such that $K \subset \operatorname{int}\left(T_{S}(\bar{x})\right) \cup\{0\}$. Then there exists a scalar $\lambda>0$ such that $K \cap \mathbb{B} \subset \lambda(\operatorname{int}(S)-\bar{x}) \cup\{0\}$.

Proof. Let us denote by $\mathbb{S}$ the closed unit sphere and let us show that $K \cap \mathbb{S} \subset$ $\lambda(\operatorname{int}(S)-\bar{x})$ for some $\lambda>0$. Let us argue by contradiction and assume that there exists a sequence $\left(d_{n}\right)$ such that $d_{n} \in K \cap \mathbb{S}$ and $d_{n} \notin n(\operatorname{int}(S)-\bar{x})$. Since the set $K \cap \mathbb{S}$ is compact, there exist $d \in K \cap \mathbb{S}$ along with a subsequence of $\left(d_{n}\right)$, still denoted by $\left(d_{n}\right)$, such that $\lim _{n \rightarrow+\infty} d_{n}=d$. By assumption, we have $K \subset \operatorname{int}\left(T_{S}(\bar{x})\right) \cup\{0\}$, whence $d \in K \cap \mathbb{S} \subset \operatorname{int}\left(T_{S}(\bar{x})\right)$. Recalling that $\operatorname{int}\left(T_{S}(\bar{x})\right)=\bigcup_{\lambda>0} \lambda(\operatorname{int}(S)-\bar{x})$, there exists $\lambda>0$ such that $d \in \lambda(\operatorname{int}(S)-\bar{x})$. Since the set $\lambda(\operatorname{int}(S)-\bar{x})$ is open, there exists $n_{0} \in \mathbb{N}$ such that $d_{n} \in \lambda(\operatorname{int}(S)-\bar{x})$ for every $n \geq n_{0}$. By taking $m \geq \max \left(n_{0}, \lambda\right)$, we obtain $d_{m} \in m(\operatorname{int}(S)-\bar{x})$, a contradiction. The conclusion follows immediately.

From now on, we assume that the set $S$ coincides with the set of minima of a lower semicontinuous convex function $\Phi: \mathbb{R}^{N} \rightarrow \mathbb{R} \cup\{+\infty\}$.

Lemma 7.4. Let $\Phi: \mathbb{R}^{N} \rightarrow \mathbb{R} \cup\{+\infty\}$ be a proper lower semicontinuous convex function such that $S=\operatorname{argmin} \Phi \neq \emptyset$. Let $\bar{x} \in S$ and assume that there exists an open cone $K_{0}$ such that $N_{S}(\bar{x}) \subset K_{0} \cup\{0\}$. Then there exists a neighborhood $V$ of $\bar{x}$ such that $\partial \Phi(x) \subset K_{0} \cup\{0\}$ for every $x \in V$.

Proof. Let us argue by contradiction and assume that there exist a sequence $\left(x_{n}\right)$ tending toward $\bar{x}$ as $n \rightarrow+\infty$, along with a sequence $\left(x_{n}^{*}\right)$ such that $x_{n}^{*} \in \partial \Phi\left(x_{n}\right)$ and $x_{n}^{*} \notin K_{0} \cup\{0\}$ for every $n \in \mathbb{N}$. From the sequence $\left(x_{n}^{*} /\left\|x_{n}^{*}\right\|\right)$ we can extract a subsequence, still denoted by $\left(x_{n}^{*} /\left\|x_{n}^{*}\right\|\right)$, such that $\lim _{n \rightarrow+\infty} x_{n}^{*} /\left\|x_{n}^{*}\right\|=x^{*}$. We clearly have $x^{*} \in \lim \sup _{x \rightarrow \bar{x}} \mathbb{R}_{+} \partial \Phi(x)$ and hence $x^{*} \in N_{[\Phi \leq \Phi(\bar{x})]}(\bar{x})=N_{S}(\bar{x})$, in view of the formula (3.3) of Theorem 3.1. Recalling that $N_{S}(\bar{x}) \subset K_{0} \cup\{0\}$ by assumption and observing that $x^{*} \neq 0$, we deduce that $x^{*} \in K_{0}$. On the other hand, since $K_{0}$ is a cone, we have $x_{n}^{*} /\left\|x_{n}^{*}\right\| \in \mathbb{R}^{N} \backslash K_{0}$ for every $n \in \mathbb{N}$. Taking the limit as $n \rightarrow+\infty$, we infer that $x^{*} \in \operatorname{cl}\left(\mathbb{R}^{N} \backslash K_{0}\right)=\mathbb{R}^{N} \backslash K_{0}$, a contradiction.

By gathering Lemmas 7.1, 7.3 and 7.4, we obtain the following statement. 
Theorem 7.5. Let $\Phi: \mathbb{R}^{N} \rightarrow \mathbb{R} \cup\{+\infty\}$ be a proper lower semicontinuous convex function such that $S=\operatorname{argmin} \Phi \neq \emptyset$. Let $\bar{x} \in S$ and assume that

$$
-N_{S}(\bar{x}) \subset \operatorname{int}\left(T_{S}(\bar{x})\right) \cup\{0\} .
$$

Then there exist a scalar $\lambda>0$ and a convex cone $K \subset \mathbb{R}^{N}$ which is closed and pointed, along with a neighborhood $V$ of $\bar{x}$ such that

(7.4) $K \cap \mathbb{B} \subset \lambda(\operatorname{int}(S)-\bar{x}) \cup\{0\}$ and $-\partial \Phi(x) \subset \operatorname{int}(K) \cup\{0\} \quad$ for every $x \in V$.

Proof. Lemma 7.1 furnishes a closed convex cone $K \subset \mathbb{R}^{N}$ which is pointed and satisfies conditions (7.2)-(7.3). Lemma 7.3 then gives some $\lambda>0$ such that the first assertion of (7.4) is fulfilled. We finally use Lemma 7.4 with $K_{0}=-\operatorname{int}(K)$. We then obtain the existence of a neighborhood $V$ of $\bar{x}$ such that $\partial \Phi(x) \subset-\operatorname{int}(K) \cup\{0\}$ for every $x \in V$, which proves the second assertion of (7.4).

The result of Theorem 7.5 can already be found in [17, Lemma 4.2], but the proof given here is slightly different and relies on the tools that are developed above. In particular, Lemma 7.4 is based on the expression of $N_{S}(\bar{x})$ as an upper limit of subgradients near $\bar{x}$; see formula (3.3).

7.2. A second-order in time gradient system with vanishing damping. Given a convex function $\Phi: \mathbb{R}^{N} \rightarrow \mathbb{R}$ and a map $\gamma \in \mathcal{C}\left(\mathbb{R}_{+}, \mathbb{R}_{+}\right)$, we consider the following differential inclusion:

$$
\ddot{x}(t)+\gamma(t) \dot{x}(t)+\partial \Phi(x(t)) \ni 0, \quad t \geq 0 .
$$

Our purpose is not to develop the questions of existence and uniqueness for solutions to this dynamical system. We will assume in the sequel that there exists a solution $x \in W_{\text {loc }}^{2,1}\left(\mathbb{R}_{+}, \mathbb{R}^{N}\right)$ satisfying $(E)$ almost everywhere.

The decay properties of the function $\gamma$ play a central role in the asymptotic behavior of $(E)$. In particular, if the quantity $\gamma(t)$ tends to 0 too rapidly as $t \rightarrow+\infty$, convergence of the trajectory may fail (think about the extreme case of $\gamma \equiv 0$ for instance). When $\int_{0}^{+\infty} \gamma(t) d t=+\infty$ and $\operatorname{argmin} \Phi \neq \emptyset$, it can be easily proved that $\lim _{t \rightarrow+\infty} \Phi(x(t))=\min \Phi$; see [15, 18, where energy-like arguments are used. In the case of a unique minimum $\bar{x}$, this immediately implies that the solution $x(t)$ converges toward $\bar{x}$ as $t \rightarrow+\infty$. The situation is much more complicated when the function $\Phi$ has a continuum of minima. Let us first consider the particular case $\Phi \equiv 0$. The differential inclusion $(E)$ then becomes $\ddot{x}(t)+\gamma(t) \dot{x}(t)=0$ and a double integration immediately shows that its solution is given by

$$
x(t)=x(0)+\dot{x}(0) \int_{0}^{t} e^{-\int_{0}^{s} \gamma(u) d u} d s .
$$

If $\dot{x}(0) \neq 0$, the solution $x(\cdot)$ converges if and only if the quantity $\int_{0}^{\infty} e^{-\int_{0}^{s} \gamma(u) d u} d s$ is finite.

Coming back to the general case of a convex function $\Phi: \mathbb{R}^{N} \rightarrow \mathbb{R}$ with a nonempty set $S$ of minima, the next result shows that if $\int_{0}^{\infty} e^{-\int_{0}^{s} \gamma(u) d u} d s=+\infty$, then nonstationary solutions of $(E)$ cannot converge toward a minimum point $\bar{x} \in S$ satisfying the obtuseness condition

$$
-N_{S}(\bar{x}) \subset \operatorname{int}\left(T_{S}(\bar{x})\right) \cup\{0\} .
$$

This is a generalization of [15, Theorem 4.1]. 
Theorem 7.6. Let $\Phi: \mathbb{R}^{N} \rightarrow \mathbb{R}$ be a convex function and let $\bar{x} \in S=\operatorname{argmin} \Phi$ satisfying the obtuseness condition (7.6). Let $\gamma \in \mathcal{C}\left(\mathbb{R}_{+}, \mathbb{R}_{+}\right)$be a function such that $\int_{0}^{\infty} e^{-\int_{0}^{s} \gamma(u) d u} d s=+\infty$. Assume that there exists a solution $x \in W_{\text {loc }}^{2,1}\left(\mathbb{R}_{+}, \mathbb{R}^{N}\right)$ satisfying equation $(E)$ almost everywhere on $[0,+\infty[$. If the map $x(\cdot)$ converges toward $\bar{x}$, then it is stationary.

Proof. Assume that $\lim _{t \rightarrow+\infty} x(t)=\bar{x}$. From Theorem 7.5 , there exist a convex cone $K \subset \mathbb{R}^{N}$, which is closed and pointed, along with $\lambda>0$ and $t_{0} \geq 0$ such that

$$
K \cap \frac{1}{\lambda} \mathbb{B} \subset(\operatorname{int}(S)-\bar{x}) \cup\{0\} \quad \text { and } \quad-\partial \Phi(x(t)) \subset K \quad \text { for every } t \geq t_{0} .
$$

Let $v \in K^{*}$. Observing that for almost every $t \geq t_{0}$

$$
\ddot{x}(t)+\gamma(t) \dot{x}(t) \in-\partial \Phi(x(t)) \subset K,
$$

we deduce that

$$
\langle\ddot{x}(t)+\gamma(t) \dot{x}(t), v\rangle \leq 0 \quad \text { a.e. on }\left[t_{0},+\infty[\text {. }\right.
$$

Let us define the function $p \in W_{l o c}^{2,1}\left(\mathbb{R}_{+}, \mathbb{R}\right)$ by $p(t)=\langle x(t), v\rangle$. In view of the above inequality, the function $p$ satisfies

$$
\ddot{p}(t)+\gamma(t) \dot{p}(t) \leq 0 \quad \text { a.e. on }\left[t_{0},+\infty[.\right.
$$

Let us prove that $\dot{p}(t)=\langle\dot{x}(t), v\rangle \geq 0$ for every $t \geq t_{0}$. Let us argue by contradiction and assume that there exists $t_{1} \geq t_{0}$ such that $\dot{p}\left(t_{1}\right)<0$. Let us multiply inequality (17.8) by $e^{\int_{t_{1}}^{t} \gamma(s) d s}$ and integrate on $\left[t_{1}, t\right]$. Recalling that $\dot{p} \in W_{\text {loc }}^{1,1}\left(\mathbb{R}_{+}, \mathbb{R}\right)$, we obtain

$$
\forall t \geq t_{1}, \quad \dot{p}(t) \leq \dot{p}\left(t_{1}\right) e^{-\int_{t_{1}}^{t} \gamma(s) d s} .
$$

By integrating again, we find

$$
\forall t \geq t_{1}, \quad p(t) \leq p\left(t_{1}\right)+\dot{p}\left(t_{1}\right) \int_{t_{1}}^{t} e^{-\int_{t_{1}}^{s} \gamma(u) d u} d s .
$$

Since $\int_{0}^{+\infty} e^{-\int_{0}^{s} \gamma(u) d u} d s=+\infty$ and $\dot{p}\left(t_{1}\right)<0$ by assumption, we deduce that $\lim _{t \rightarrow+\infty} p(t)=-\infty$. This contradicts the boundedness of the map $x(\cdot)$, hence we conclude that

$$
\forall t \geq t_{0}, \quad\langle\dot{x}(t), v\rangle \geq 0 .
$$

By integrating the above inequality on $\left[t,+\infty\left[\right.\right.$ and using the fact that $\lim _{t \rightarrow+\infty} x(t)$ $=\bar{x}$, we obtain $\langle x(t)-\bar{x}, v\rangle \leq 0$ for every $t \geq t_{0}$. Since this is true for every $v \in K^{*}$, we derive that $x(t)-\bar{x} \in K^{* *}$ for every $t \geq t_{0}$. Recalling that $K^{* *}=K$ for every closed convex cone $K$, we conclude that

$$
\forall t \geq t_{0}, \quad x(t)-\bar{x} \in K .
$$

By again using $\lim _{t \rightarrow+\infty} x(t)=\bar{x}$, we find the existence of $t_{1} \geq t_{0}$ such that $x(t)-\bar{x} \in \frac{1}{\lambda} \mathbb{B}$ for every $t \geq t_{1}$. In view of (7.7), we infer that $x(t) \in \operatorname{int}(S) \cup\{\bar{x}\}$ for every $t \geq t_{1}$. Let us now distinguish the following cases:

(a) For every $t \geq t_{1}$, we have $x(t) \in \operatorname{int}(S)$.

(b) There exists $t_{2} \geq t_{1}$ such that $x\left(t_{2}\right)=\bar{x}$. 
Case (a). We then have $\partial \Phi(x(t))=\{0\}$ for every $t \geq t_{1}$, so that equation $(E)$ becomes

$$
\ddot{x}(t)+\gamma(t) \dot{x}(t)=0 \quad \text { a.e. on }\left[t_{1},+\infty[\text {. }\right.
$$

Let us prove that $\dot{x}(t)=0$ for every $t \geq t_{1}$. Let us argue by contradiction and assume that there exists $t_{1}^{*} \geq t_{1}$ such that $\dot{x}\left(t_{1}^{*}\right) \neq 0$. The same computation as in (7.5) shows that

$$
\forall t \geq t_{1}^{*}, \quad x(t)=x\left(t_{1}^{*}\right)+\dot{x}\left(t_{1}^{*}\right) \int_{t_{1}^{*}}^{t} e^{-\int_{t_{1}^{*}}^{s} \gamma(u) d u} d s .
$$

Since $\int_{0}^{+\infty} e^{-\int_{0}^{s} \gamma(u) d u} d s=+\infty$ and $\dot{x}\left(t_{1}^{*}\right) \neq 0$ by assumption, we deduce that $\lim _{t \rightarrow+\infty}|x(t)|=+\infty$. This contradicts the boundedness of the map $x(\cdot)$, hence we conclude that $\dot{x}(t)=0$ for every $t \geq t_{1}$, thus implying that $x(t)=x\left(t_{1}\right)$ for every $t \geq t_{1}$.

Case (b). Since $x\left(t_{2}\right)=\bar{x}$ by assumption, the integration of inequality (17.9) on $\left[t_{2}, t\right]$ gives $\langle x(t)-\bar{x}, v\rangle \geq 0$ for every $t \geq t_{2}$. By arguing as above, we obtain that $x(t)-\bar{x} \in-K$ for every $t \geq t_{2}$. In view of (7.10), we infer that

$$
\forall t \geq t_{2}, \quad x(t)-\bar{x} \in K \cap(-K) .
$$

Recalling that the cone $K$ is pointed, we have $K \cap(-K)=\{0\}$, hence $x(t)=\bar{x}$ for every $t \geq t_{2}$.

As a conclusion, we have proved in both cases $(a)$ and $(b)$ that the solution $x(\cdot)$ is stationary, which ends the proof.

\section{REFERENCES}

[1] F. Alvarez, On the minimizing property of a second order dissipative system in Hilbert spaces, SIAM J. Control Optim. 38 (2000), no. 4, 1102-1119 (electronic), DOI 10.1137/S0363012998335802. MR1760062 (2001e:34118)

[2] H. Attouch, Variational convergence for functions and operators, Applicable Mathematics Series, Pitman (Advanced Publishing Program), Boston, MA, 1984. MR773850 (86f:49002)

[3] H. Attouch, J.-B. Baillon, and M. Théra, Variational sum of monotone operators, J. Convex Anal. 1 (1994), no. 1, 1-29. MR1326939 (97a:47086)

[4] H. Attouch, G. Buttazzo, and G. Michaille, Variational analysis in Sobolev and BV spaces, MPS/SIAM Series on Optimization, vol. 6, Society for Industrial and Applied Mathematics (SIAM), Philadelphia, PA, 2006. Applications to PDEs and optimization. MR2192832 (2006j:49001)

[5] H. Attouch, A. Cabot, and P. Redont, The dynamics of elastic shocks via epigraphical regularization of a differential inclusion. Barrier and penalty approximations, Adv. Math. Sci. Appl. 12 (2002), no. 1, 273-306. MR1909449 (2003e:34017)

[6] H. Attouch, X. Goudou, and P. Redont, The heavy ball with friction method. I. The continuous dynamical system: global exploration of the local minima of a real-valued function by asymptotic analysis of a dissipative dynamical system, Commun. Contemp. Math. 2 (2000), no. 1, 1-34, DOI 10.1142/S0219199700000025. MR.1753136 (2001b:37025)

[7] A. Auslender and M. Teboulle, Asymptotic cones and functions in optimization and variational inequalities, Springer Monographs in Mathematics, Springer-Verlag, New York, 2003. MR.1931309 (2003i:49002)

[8] D. Azé, Eléments d'analyse convexe et variationnelle, Ellipses, Paris, 1997.

[9] J. F. Bonnans and A. Shapiro, Perturbation analysis of optimization problems, Springer Series in Operations Research, Springer-Verlag, New York, 2000. MR1756264 (2001g:90003)

[10] J. M. Borwein, A note on $\varepsilon$-subgradients and maximal monotonicity, Pacific J. Math. 103 (1982), no. 2, 307-314. MR705231 (85h:90091) 
[11] J. M. Borwein and A. S. Lewis, Convex analysis and nonlinear optimization, CMS Books in Mathematics/Ouvrages de Mathématiques de la SMC, 3, Springer-Verlag, New York, 2000. Theory and examples. MR.1757448 (2001h:49001)

[12] M. Bounkhel and L. Thibault, On various notions of regularity of sets in nonsmooth analysis, Nonlinear Anal. 48 (2002), no. 2, Ser. A: Theory Methods, 223-246, DOI 10.1016/S0362546X(00)00183-8. MR.1870754 (2002j:46048)

[13] N. Bourbaki, Éléments de mathématique. Fasc. XV. Livre V: Espaces vectoriels topologiques. Chapitre I: Espaces vectoriels topologiques sur un corps valué. Chapitre II: Ensembles convexes et espaces localement convexes (French), Actualités Scientifiques et Industrielles, No. 1189. Deuxième édition revue et corrigée, Hermann, Paris, 1966. MR0203425 (34 \#3277)

[14] A. Brøndsted and R. T. Rockafellar, On the subdifferentiability of convex functions, Proc. Amer. Math. Soc. 16 (1965), 605-611. MR0178103 (31 \#2361)

[15] A. Cabot, H. Engler, and S. Gadat, On the long time behavior of second order differential equations with asymptotically small dissipation, Trans. Amer. Math. Soc. 361 (2009), no. 11, 5983-6017, DOI 10.1090/S0002-9947-09-04785-0. MR2529922 (2010m:34094)

[16] A. Cabot, H. Engler, and S. Gadat, Second-order differential equations with asymptotically small dissipation and piecewise flat potentials, Proceedings of the Seventh Mississippi StateUAB Conference on Differential Equations and Computational Simulations, Electron. J. Differ. Equ. Conf., vol. 17, Southwest Texas State Univ., San Marcos, TX, 2009, pp. 33-38. MR2605582(2011d:34096)

[17] A. Cabot and P. Frankel, Asymptotics for some proximal-like method involving inertia and memory aspects, Set-Valued Var. Anal. 19 (2011), no. 1, 59-74, DOI 10.1007/s11228-0100140-1. MR2770897 (2011m:65123)

[18] A. Cabot and P. Frankel, Asymptotics for some semilinear hyperbolic equations with non-autonomous damping, J. Differential Equations 252 (2012), no. 1, 294-322, DOI 10.1016/j.jde.2011.09.012. MR.2852207

[19] A. Cabot and L. Paoli, Asymptotics for some vibro-impact problems with a linear dissipation term (English, with English and French summaries), J. Math. Pures Appl. (9) 87 (2007), no. 3, 291-323, DOI 10.1016/j.matpur.2007.01.003. MR.2312513 (2008e:34024)

[20] F. H. Clarke, Optimization and nonsmooth analysis, Canadian Mathematical Society Series of Monographs and Advanced Texts, John Wiley \& Sons Inc., New York, 1983. A WileyInterscience Publication. MR709590 (85m:49002)

[21] F. H. Clarke, Y. S. Ledyaev, R. J. Stern, and P. R. Wolenski, Nonsmooth analysis and control theory, Graduate Texts in Mathematics, vol. 178, Springer-Verlag, New York, 1998. MR $1488695(99 \mathrm{a}: 49001)$

[22] J.-B. Hiriart-Urruty, New concepts in nondifferentiable programming, Bull. Soc. Math. France Mém. 60 (1979), 57-85. Analyse non convexe (Proc. Colloq., Pau, 1977). MR562256 (81a:90138)

[23] J.-B. Hiriart-Urruty and C. Lemaréchal, Convex analysis and minimization algorithms. I, Grundlehren der Mathematischen Wissenschaften [Fundamental Principles of Mathematical Sciences], vol. 305, Springer-Verlag, Berlin, 1993. Fundamentals. MR1261420 (95m:90001)

[24] J.-B. Hiriart-Urruty, M. Moussaoui, A. Seeger, and M. Volle, Subdifferential calculus without qualification conditions, using approximate subdifferentials: a survey, Nonlinear Anal. 24 (1995), no. 12, 1727-1754, DOI 10.1016/0362-546X(94)00221-3. MR.1330645 (96a:49022)

[25] J.-B. Hiriart-Urruty and R. R. Phelps, Subdifferential calculus using $\epsilon$-subdifferentials, J. Funct. Anal. 118 (1993), no. 1, 154-166, DOI 10.1006/jfan.1993.1141. MR.1245600 (94h:49029)

[26] F. Jules and M. Lassonde, Formulas for subdifferentials of sums of convex functions, J. Convex Anal. 9 (2002), no. 2, 519-533. Special issue on optimization (Montpellier, 2000). MR.1970570 (2004b:49029)

[27] F. Mignot, Contrôle dans les inéquations variationelles elliptiques (French), J. Functional Analysis 22 (1976), no. 2, 130-185. MR0423155 (54 \#11136)

[28] B. S. Mordukhovich, Variational analysis and generalized differentiation. I, Grundlehren der Mathematischen Wissenschaften [Fundamental Principles of Mathematical Sciences], vol. 330, Springer-Verlag, Berlin, 2006. Basic theory. MR2191744 (2007b:49003a)

[29] B. S. Mordukhovich and Y. Shao, Fuzzy calculus for coderivatives of multifunctions, Nonlinear Anal. 29 (1997), no. 6, 605-626, DOI 10.1016/S0362-546X(96)00082-X. MR1452749 (98m:49038) 
[30] J.-P. Penot, Subdifferential calculus without qualification assumptions, J. Convex Anal. 3 (1996), no. 2, 207-219. MR.1448052(2000d:49030)

[31] R. T. Rockafellar, Convex analysis, Princeton Mathematical Series, No. 28, Princeton University Press, Princeton, N.J., 1970. MR0274683 (43 \#445)

[32] R. T. Rockafellar and R. J.-B. Wets, Variational analysis, Grundlehren der Mathematischen Wissenschaften [Fundamental Principles of Mathematical Sciences], vol. 317, Springer-Verlag, Berlin, 1998. MR1491362 (98m:49001)

[33] L. Thibault, A generalized sequential formula for subdifferentials of sums of convex functions defined on Banach spaces, Recent developments in optimization (Dijon, 1994), Lecture Notes in Econom. and Math. Systems, vol. 429, Springer, Berlin, 1995, pp. 340-345, DOI 10.1007/978-3-642-46823-0_25. MR.1358408 (96h:49041)

[34] L. Thibault, Sequential convex subdifferential calculus and sequential Lagrange multipliers, SIAM J. Control Optim. 35 (1997), no. 4, 1434-1444, DOI 10.1137/S0363012995287714. MR1453305 (98f:49020)

[35] L. Thibault, Limiting convex subdifferential calculus with applications to integration and maximal monotonicity of subdifferential, Constructive, experimental, and nonlinear analysis (Limoges, 1999), CMS Conf. Proc., vol. 27, Amer. Math. Soc., Providence, RI, 2000, pp. 279289. MR.1777630 (2001g:49019)

Département de Mathématiques, Université Montpellier II, CC 051, Place Eugène Bataillon, 34095 Montpellier Cedex 5, France

E-mail address: acabot@math.univ-montp2.fr

Département de Mathématiques, Université Montpellier II, CC 051, Place Eugène Bataillon, 34095 Montpellier Cedex 5, France

E-mail address: thibault@math.univ-montp2.fr

Current address: Centro de Modelamiento Matemático (CMM), Universidad de Chile, Santiago, Chile 